\title{
A Total Synthesis of Paeoveitol
}

\author{
Lun $\mathrm{Xu},{ }^{\S}$ Fengyi Liu, ${ }^{\S}$ Li-Wen Xu, Ziwei Gao, Yu-Ming Zhao*
}

Key Laboratory of Applied Surface and Colloid Chemistry of MOE \& School of Chemistry and Chemical

Engineering, Shaanxi Normal University

620 West Chang'an Ave, Xi'an, 710119, China

ymzhao@snnu.edu.cn

\section{Table of contents}

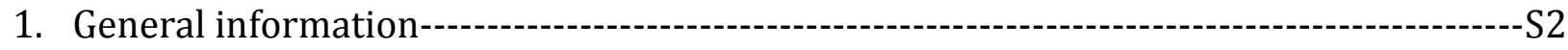

2. Experimental Procedures---ons

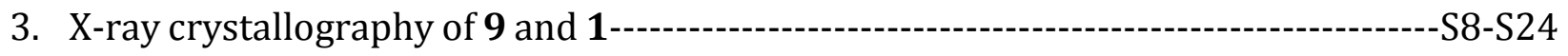

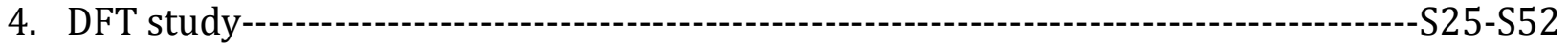

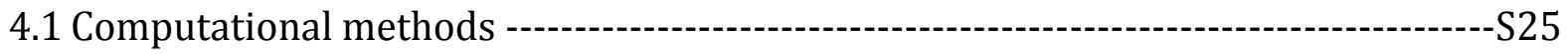

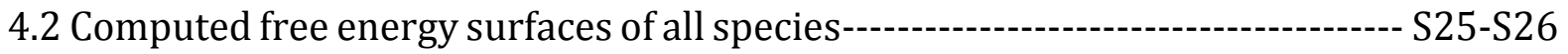

4.3 Cartesian computed coordinates and Gibbs free energies of all species---------S27-S52

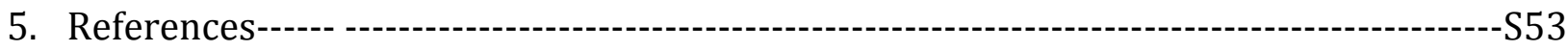

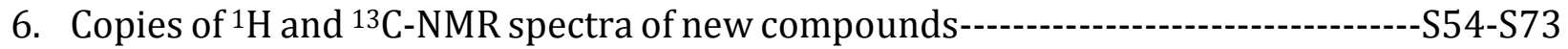




\section{General information}

Unless otherwise stated, all reactions were performed in oven-dried or flame-dried glassware under an atmosphere of dry nitrogen. Anhydrous dichloromethane was purified by distillation over calcium hydride. Anhydrous tetrahydrofuran was freshly distilled from sodium-benzophenone. Reactions were monitored by thin layer chromatography (TLC) (250 $\mu \mathrm{m}$ thickness, F-254 indicator) and visualized by UV irradiation and staining with $p$ anisaldehyde, phosphomolybdic acid, or potassium permanganate developing agents. Volatile solvents were removed under reduced pressure using a rotary evaporator. Flash column chromatography was performed over silica gel (230-400 mesh) purchased from Qindao Haiyang Co., China. ${ }^{1} \mathrm{H}$ and ${ }^{13} \mathrm{C}$ NMR spectra were recorded on a Bruker AV $600 \mathrm{MHz}$ NMR spectrometer using residue solvent peaks as an internal standard. Chemical shifts are reported in parts per million ( $\mathrm{ppm})$ with respect to the residual solvent signal. Peak multiplicities are reported as follows: $\mathrm{s}=$ singlet, $\mathrm{bs}=$ broad singlet, $\mathrm{d}=$ doublet, $\mathrm{t}=$ triplet, $\mathrm{dd}=$ doublet of doublets, $\mathrm{td}=$ triplet of doublets, $\mathrm{m}=$ multiplet. app = apparent. Melting points were determined using MEl-TEMP ${ }^{\mathrm{TM}}$ apparatus and are uncorrected. IR spectra were recorded on a Nicollet 170SX FT-IR spectrometer. High-resolution mass spectra (HRMS) were collected on a Bruker Maxis System. X-ray crystallographic analyses were performed on Bruker D8 Quest. 


\section{Experimental Procedures}

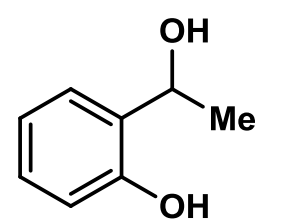

2-(1-hydroxyethyl)phenol (6). $\mathrm{NaBH}_{4}$ (278 mg, $7.4 \mathrm{mmol}, 0.5$ equiv) was added to a solution of o-acetylphenol (2.0 g, $14.8 \mathrm{mmol}, 1.0$ equiv $)$ in anhydrous methanol $(20 \mathrm{~mL})$ over a period of $5 \mathrm{~min}$ at $0{ }^{\circ} \mathrm{C}$. The reaction mixture was slowly warmed to room temperature and stirred for an additional 1 hour. Cold water $(10 \mathrm{~mL})$ was slowly added at $0{ }^{\circ} \mathrm{C}$ and the reaction mixture stirred for $15 \mathrm{~min}$. The aqueous phase was extracted with ethyl acetate $(2 \times 100 \mathrm{~mL})$. The combined organic phases were washed with brine ( $50 \mathrm{~mL})$, dried over $\mathrm{Na}_{2} \mathrm{SO}_{4}$, and concentrated in vacuo. The residue was purified by flash column chromatography $\left(\mathrm{SiO}_{2}\right.$, ethyl acetate/hexane) gave 6 (1.9 g, 94 \%) as a colorless oil: ${ }^{1} \mathrm{H}$ NMR (600 MHz, $\left.\mathrm{CDCl}_{3}\right) \delta 8.06(\mathrm{~s}, 1 \mathrm{H}), 7.15(\mathrm{~m}, 1 \mathrm{H}), 6.96(\mathrm{dd}, J=7.5$, $1.3 \mathrm{~Hz}, 1 \mathrm{H}), 6.90-6.78(\mathrm{~m}, 2 \mathrm{H}), 5.10-4.96(\mathrm{~m}, 1 \mathrm{H}), 3.07$ (d, J = 3.9 Hz, 1H), 1.54 (d, J = 6.6 $\mathrm{Hz}, 3 \mathrm{H}) ;{ }^{13} \mathrm{C}$ NMR $\left(151 \mathrm{MHz}, \mathrm{CDCl}_{3}\right) \delta 155.2,128.9,128.6,126.5,120.0,117.0,71.4,23.4$; IR (thin film, $\mathrm{cm}^{-1}$ ) 3320, 2980, 2845, 1600, 1500, 1450, 1020, 760; HRMS (ESI) calcd. for $\left[\mathrm{C}_{8} \mathrm{H}_{10} \mathrm{O}_{2} \mathrm{Na}\right]^{+}(\mathrm{M}+\mathrm{Na})^{+}: \mathrm{m} / z$ 161.0573, found 161.0579.

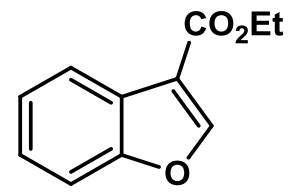

Ethyl benzofuran-3-carboxylate (4). To a solution of salicyaldehyde (2.0 g, $16.4 \mathrm{mmol}, 1.0$ equiv] and $\mathrm{HBF}_{4} \bullet \mathrm{Et}_{2} \mathrm{O}$ [0.3 ml, $1.64 \mathrm{mmol}, 0.1$ equiv; $54 \%$ $(\mathrm{w} / \mathrm{w})]$ in $\mathrm{CH}_{2} \mathrm{Cl}_{2}(10 \mathrm{~mL})$ at room temperature was added ethyl diazoacetate ( $2.6 \mathrm{~mL}, 24.6 \mathrm{mmol}, 1.5$ equiv) via syringe pump over $30 \mathrm{~min}$ at $5{ }^{\circ} \mathrm{C}$. The reaction mixture was stirred for another $30 \mathrm{~min}$ at room temperature and concentrated in vacuo. Concentrated sulfuric acid $(1.5 \mathrm{~mL})$ was added and stirred for $30 \mathrm{~min}$ at room temperature. The reaction mixture was diluted with $\mathrm{CH}_{2} \mathrm{Cl}_{2}(50 \mathrm{~mL})$ and the excess of acid was carefully quenched with saturated aqueous $\mathrm{NaHCO}_{3}$ solution. The organic layer was separated and the aqueous layer was extracted with $\mathrm{CH}_{2} \mathrm{Cl}_{2}(2 \times 50 \mathrm{~mL})$. The combined organic phase was washed with brine $(40 \mathrm{~mL})$, dried over $\mathrm{Na}_{2} \mathrm{SO}_{4}$, and concentrated in vacuo. The residue was purified by flash column chromatography $\left(\mathrm{SiO}_{2}\right.$, ethyl acetate/hexane) gave $4(2.8 \mathrm{~g}, 90 \%)$ as a colorless liquid: ${ }^{1} \mathrm{H}$ NMR $\left(600 \mathrm{MHz}, \mathrm{CDCl}_{3}\right) \delta 8.24(\mathrm{~s}, 1 \mathrm{H}), 8.10-8.04(\mathrm{~m}$, 1H), $7.54-7.49(\mathrm{~m}, 1 \mathrm{H}), 7.38-7.32(\mathrm{~m}, 2 \mathrm{H}), 4.40(\mathrm{q}, J=7.2 \mathrm{~Hz}, 2 \mathrm{H}), 1.41(\mathrm{t}, J=7.2 \mathrm{~Hz}, 3 \mathrm{H})$; ${ }^{13} \mathrm{C}$ NMR $\left(151 \mathrm{MHz}, \mathrm{CDCl}_{3}\right) \delta 163.4,155.6,150.9,125.2,124.7,124.1,122.1,114.8,111.7$, 
60.5, 14.4; IR (thin film, $\mathrm{cm}^{-1}$ ) 2975, 2952, 2840, 1726, 1600, 1563, 1450, 1105, 1021, 754; HRMS (ESI) calcd. for $\left[\mathrm{C}_{11} \mathrm{H}_{10} \mathrm{O}_{3} \mathrm{Na}\right]^{+}(\mathrm{M}+\mathrm{Na})^{+}: \mathrm{m} / z$ 213.0522, found 213.0525 .

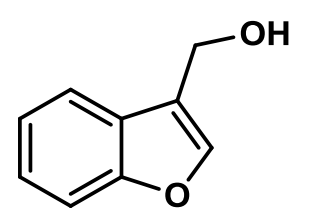

Benzofuran-3-ylmethanol (5). To a solution of ester 4 (2.0 g, $10.5 \mathrm{mmol}$, 1.0 equiv) in $\mathrm{CH}_{2} \mathrm{Cl}_{2}(30 \mathrm{~mL})$ at $-78{ }^{\circ} \mathrm{C}$ was added a solution of diisobutylaluminium hydride ( $17.5 \mathrm{~mL}, 26.3 \mathrm{mmol}$, 2.5 equiv) in toluene $(1.5 \mathrm{M})$ via syringe. The reaction mixture was slowly warmed to room temperature and stirred for 1 hour. The reaction was quenched with saturated aqueous $\mathrm{NH}_{4} \mathrm{Cl}$ solution. The organic layer was separated and the aqueous layer was extracted with $\mathrm{CH}_{2} \mathrm{Cl}_{2}(100 \mathrm{~mL})$. The combined organic phase was washed with brine, dried over $\mathrm{Na}_{2} \mathrm{SO}_{4}$, and concentrated in vacuo. The residue was purified by flash column chromatography $\left(\mathrm{SiO}_{2}\right.$, ethyl acetate/hexane) gave 5 (1.4 g, 90\%) as a white solid: m.p. $=45-47{ }^{\circ} \mathrm{C} ;{ }^{1} \mathrm{H}$ NMR $(600 \mathrm{MHz}$, $\left.\mathrm{CDCl}_{3}\right) \delta 7.64(\mathrm{~d}, J=7.7 \mathrm{~Hz}, 1 \mathrm{H}), 7.57(\mathrm{~s}, 1 \mathrm{H}), 7.48(\mathrm{~d}, J=8.2 \mathrm{~Hz}, 1 \mathrm{H}), 7.34-7.29(\mathrm{~m}, 1 \mathrm{H}), 7.28$ - $7.22(\mathrm{~m}, 1 \mathrm{H}), 4.79(\mathrm{~s}, 2 \mathrm{H}), 1.96(\mathrm{~s}, 1 \mathrm{H}) ;{ }^{13} \mathrm{C} \mathrm{NMR}\left(151 \mathrm{MHz}, \mathrm{CDCl}_{3}\right) \delta$ 155.6, 142.4, 126.7, 124.7, 122.8, 120.4, 120.0, 111.6, 55.9; IR (thin film, $\mathrm{cm}^{-1}$ ) 3420, 2950, 2840, 1640, 1450, 1034, 750; HRMS (ESI) calcd. for $\left[\mathrm{C}_{9} \mathrm{H}_{8} \mathrm{O}_{2} \mathrm{Na}\right]^{+}(\mathrm{M}+\mathrm{Na})^{+}: \mathrm{m} / z$ 171.0417, found 171.0422 .

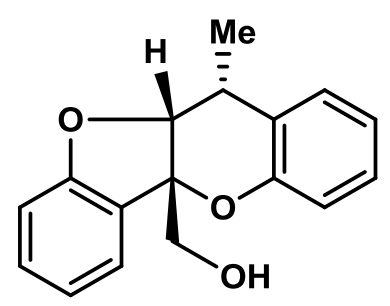

Tetracyclic alcohol 7. A solution of 5 ( $44.7 \mathrm{mg}, 0.30 \mathrm{mmol}, 1.0$ equiv) and 6 (50 mg, $0.36 \mathrm{mmol}, 1.2$ equiv) in $\mathrm{CH}_{2} \mathrm{Cl}_{2}(4 \mathrm{~mL}$ ) was treated with anhydrous $\mathrm{ZnCl}_{2}(8.5 \mathrm{mg}, 0.06 \mathrm{mmol}, 0.2$ equiv) at room temperature. After 12 hours, the reaction mixture was quenched with water $(2 \mathrm{~mL})$ and extracted with $\mathrm{CH}_{2} \mathrm{Cl}_{2}(25 \mathrm{~mL})$. The organic phase was washed with brine, dried over $\mathrm{Na}_{2} \mathrm{SO}_{4}$, and concentrated in vacuo. The residue was purified by flash column chromatography ( $\mathrm{SiO}_{2}$, ethyl acetate/hexane) gave 7 (69.2 $\left.\mathrm{mg}, 86 \%\right)$ as a White foam: m.p. $=125-127{ }^{\circ} \mathrm{C} ;{ }^{1} \mathrm{H} \mathrm{NMR}\left(600 \mathrm{MHz}, \mathrm{CDCl}_{3}\right) \delta 7.28(\mathrm{~d}, J=7.4 \mathrm{~Hz}, 1 \mathrm{H}), 7.16(\mathrm{~d}, J=7.5$ Hz, 1H), $7.08(\mathrm{~m}, 1 \mathrm{H}), 6.98(\mathrm{~m}, 1 \mathrm{H}), 6.92(\mathrm{~m}, 1 \mathrm{H}), 6.80(\mathrm{~m}, 1 \mathrm{H}), 6.67$ (d, J = 7.8 Hz, 1H), 6.57 $(\mathrm{d}, J=8.1 \mathrm{~Hz}, 1 \mathrm{H}), 5.11(\mathrm{~d}, J=3.1 \mathrm{~Hz}, 1 \mathrm{H}), 4.28(\mathrm{dd}, J=11.8,5.0 \mathrm{~Hz}, 1 \mathrm{H}), 3.95(\mathrm{dd}, J=11.8,8.8$ $\mathrm{Hz}, 1 \mathrm{H}), 3.11-3.07(\mathrm{~m}, 1 \mathrm{H}), 2.52-2.55(\mathrm{~m}, 1 \mathrm{H}), 1.60$ (d, J = 7.1 Hz, 3H); ${ }^{13} \mathrm{C}$ NMR $(151 \mathrm{MHz}$, $\left.\mathrm{CDCl}_{3}\right) \delta 160.7,154.0,131.2,128.8,127.4,125.9,125.9,124.4,122.9,120.7,117.9,110.1$, 89.4, 88.3, 67.5, 33.0, 13.2; IR (thin film, $\mathrm{cm}^{-1}$ ) 3425, 2930, 2840, 1610, 1480, 1233, 1030, 757; HRMS (ESI) calcd. for [ $\left.\mathrm{C}_{17} \mathrm{H}_{16} \mathrm{O}_{3} \mathrm{Na}\right]^{+}(\mathrm{M}+\mathrm{Na})^{+}: \mathrm{m} / z$ 291.0992, found 291.0997 . 


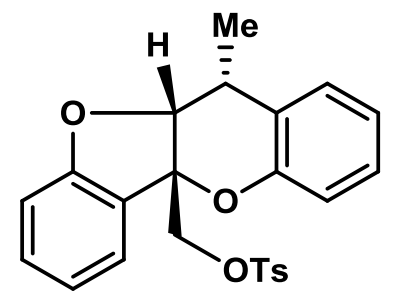

Tetracyclic Ts-ester 9. To a solution of tetracyclic alcohol 7 (50mg, $0.186 \mathrm{mmol}, 1.0$ equiv) in $\mathrm{CH}_{2} \mathrm{Cl}_{2}(2 \mathrm{~mL})$ was added $\mathrm{Et}_{3} \mathrm{~N}(80 \mathrm{uL}, 0.57$ mmol, 3.0 equiv), TsCl ( $53 \mathrm{mg}, 0.28 \mathrm{mmol}, 1.5$ equiv), and DMAP ( 2.3 $\mathrm{mg}, 0.019 \mathrm{mmol}, 0.1$ equiv) at $0{ }^{\circ} \mathrm{C}$. The reaction mixture was warmed to room temperature and stirred overnight before water was added $(1 \mathrm{~mL})$. The layers were separated, and the aqueous layer was extracted with $\mathrm{CH}_{2} \mathrm{Cl}_{2}(15 \mathrm{~mL})$. The combined organic phase was washed with brine, dried over $\mathrm{Na}_{2} \mathrm{SO}_{4}$, and concentrated in vacuo. The residue was purified by flash column chromatography $\left(\mathrm{SiO}_{2}\right.$, ethyl acetate/hexane) gave Ts-ester 9 (73.9 mg, 94\%) as a white solid : m.p.=95-97 ${ }^{\circ} \mathrm{C}$; ${ }^{1} \mathrm{H}$ NMR $\left(600 \mathrm{MHz}, \mathrm{CDCl}_{3}\right) \delta 7.77(\mathrm{~d}, J=8.3 \mathrm{~Hz}, 2 \mathrm{H}), 7.30(\mathrm{~d}, J=8.0 \mathrm{~Hz}, 2 \mathrm{H}), 7.19-7.12(\mathrm{~m}, 2 \mathrm{H}), 7.09$ (m, 1H), $6.96(\mathrm{~m}, 1 \mathrm{H}), 6.91(\mathrm{~m}, 1 \mathrm{H}), 6.79(\mathrm{~m}, 1 \mathrm{H}), 6.61-6.54(\mathrm{~m}, 2 \mathrm{H}), 5.02(\mathrm{~d}, J=3.2 \mathrm{~Hz}, 1 \mathrm{H})$, 4.69 (d, $J=10.5 \mathrm{~Hz}, 1 \mathrm{H}), 4.45$ (d, $J=10.5 \mathrm{~Hz}, 1 \mathrm{H}), 3.09$ (m, 1H), 2.41 (s, 3H), 1.57 (d, J = 7.1 $\mathrm{Hz}, 3 \mathrm{H}) ;{ }^{13} \mathrm{C} \mathrm{NMR}\left(151 \mathrm{MHz}, \mathrm{CDCl}_{3}\right) \delta 160.4,153.4,145.2,132.7,131.5,129.9,128.09,128.05$, $127.4,125.8$, 124.7 , 124.2 , 122.9 , 120.9 , 117.8 , 110.3 , 88.6 , 85.8 , 72.1 , 32.3 , 21.7 , 13.2; IR (thin film, cm-1) 2940, 2845, 1600, 1480, 1360, 1180, 751, 551; HRMS (ESI) calcd. for $\left[\mathrm{C}_{24} \mathrm{H}_{22} \mathrm{O}_{5} \mathrm{NaS}\right]^{+}(\mathrm{M}+\mathrm{Na})^{+}: \mathrm{m} / z$ 445.1080, found 445.1086 .<smiles>COc1cc(O)c(C=O)cc1O</smiles>

2,5-dihydroxy-4-methylbenzaldehyde 11. A solution of methylhydroquinone (500 mg, $4.03 \mathrm{mmol}, 1.0$ equiv) and hexamethylenetetramine (2.3 g, $16.12 \mathrm{mmol}, 4.0$ equiv) in $\mathrm{CF}_{3} \mathrm{COOH}(8 \mathrm{~mL})$ was heated to $80^{\circ} \mathrm{C}$ for 4 hours. The reaction mixture was diluted with $0.4 \% \mathrm{HCl}(40 \mathrm{~mL})$ and extracted with $\mathrm{CH}_{2} \mathrm{Cl}_{2}$ $(3 \times 30 \mathrm{~mL})$. The organic phase was washed with brine, dried over $\mathrm{Na}_{2} \mathrm{SO}_{4}$, and concentrated in vacuo. The residue was purified by flash column chromatography $\left(\mathrm{SiO}_{2}\right.$, ethyl acetate/hexane) gave aldehyde $\mathbf{1 1}$ (380 mg, $62 \%$ ) as a yellowish solid : m.p. $=156-158{ }^{\circ} \mathrm{C} ;{ }^{1} \mathrm{H}$ NMR (600 MHz, MeOH-d $\left.{ }^{4}\right) \delta 9.82(\mathrm{~s}, 1 \mathrm{H}), 6.94(\mathrm{~s}, 1 \mathrm{H}), 6.68$ (s, 1H), 4.89 (s, 2H), $2.22(\mathrm{~s}, 3 \mathrm{H})$; ${ }^{13} \mathrm{C}$ NMR (151 MHz, MeOH- $d^{4}$ ) $\delta 196.2,156.1,149.9,138.3,120.6,119.8,116.6,17.2$; IR (thin film, $\mathrm{cm}^{-1}$ ) 3392, 2980, 1756, 1634, 1480, 1250, 667; HRMS (ESI) calcd. for $\left[\mathrm{C}_{8} \mathrm{H}_{8} \mathrm{O}_{3} \mathrm{Na}\right]^{+}$ $(\mathrm{M}+\mathrm{Na})^{+}: \mathrm{m} / \mathrm{z}$ 175.0366, found 175.0371 . 


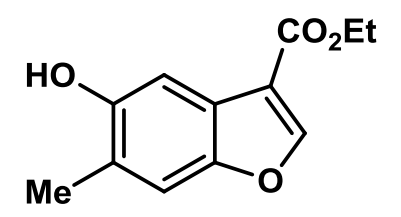

Benzofuran ester 12. To a solution of aldehyde 11 (2.0 g, 13.15 mmol, 1.0 equiv] and $\mathrm{HBF}_{4} \bullet \mathrm{Et}_{2} \mathrm{O}[0.24 \mathrm{ml}, 0.132 \mathrm{mmol}, 0.1$ equiv; $54 \%$ $(\mathrm{w} / \mathrm{w})]$ in $\mathrm{CH}_{2} \mathrm{Cl}_{2}(5 \mathrm{~mL})$ at room temperature was added a solution of ethyl diazoacetate $\left(7 \mathrm{ml}, 58 \mathrm{mmol}, 4.5\right.$ equiv) in $\mathrm{CH}_{2} \mathrm{Cl}_{2}(10 \mathrm{~mL})$ via syringe pump over $30 \mathrm{~min}$. The reaction mixture was stirred for another $30 \mathrm{~min}$ and concentrated in vacuo. Concentrated sulfuric acid (1.5 mL) was added and stirred for $30 \mathrm{~min}$ at room temperature. The reaction mixture was diluted with $\mathrm{CH}_{2} \mathrm{Cl}_{2}(50 \mathrm{~mL})$ and the excess of acid was carefully quenched with saturated aqueous $\mathrm{NaHCO}_{3}$ solution. The organic layer was separated and the aqueous layer was extracted with $\mathrm{CH}_{2} \mathrm{Cl}_{2}(2 \times 50 \mathrm{~mL})$. The combined organic phase was washed with brine $(40 \mathrm{~mL})$, dried over $\mathrm{Na}_{2} \mathrm{SO}_{4}$, and concentrated in vacuo. The residue was purified by flash column chromatography ( $\mathrm{SiO}_{2}$, ethyl acetate/hexane) gave benzofuran ester $12(2.0 \mathrm{~g}, 68 \%)$ as a white solid: $\mathrm{m} . \mathrm{p} .=150-152{ }^{\circ} \mathrm{C}$; ${ }^{1} \mathrm{H}$ NMR $\left(600 \mathrm{MHz}\right.$, acetone- $\left.d^{6}\right) \delta 8.37$ (s, 1H), $8.33(\mathrm{~s}, 1 \mathrm{H}), 7.49(\mathrm{~s}, 1 \mathrm{H}), 7.35(\mathrm{~s}, 1 \mathrm{H}), 4.35(\mathrm{q}, J=7.1 \mathrm{~Hz}, 2 \mathrm{H}), 2.34(\mathrm{~s}, 3 \mathrm{H}), 1.38(\mathrm{t}, J=$ $7.1 \mathrm{~Hz}, 3 \mathrm{H}) ;{ }^{13} \mathrm{C}$ NMR $\left(151 \mathrm{MHz}\right.$, acetone-d ${ }^{6} \delta 163.9,153.7,151.7,150.9,124.76,124.0$, 115.1, 113.5, 106.3 , 60.9, 17.0, 14.7; IR (thin film, $\mathrm{cm}^{-1}$ ) 3380,2980, 2860, 1685,1550, 1460, 1280, 1152, 780, 640; HRMS (ESI) calcd. for $\left[\mathrm{C}_{12} \mathrm{H}_{12} \mathrm{O}_{4} \mathrm{Na}\right]^{+}(\mathrm{M}+\mathrm{Na})^{+}: m / z 243.0628$, found 243.0630 .

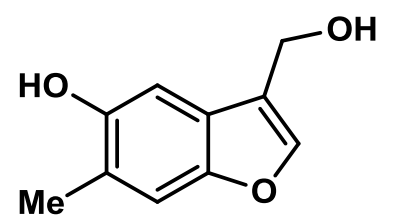

Paeoveitol D (2). To a solution of ester 12 (1.5 g, $6.8 \mathrm{mmol}, 1.0$ equiv) in $\mathrm{CH}_{2} \mathrm{Cl}_{2}(25 \mathrm{~mL})$ at $-78{ }^{\circ} \mathrm{C}$ was added a solution of diisobutylaluminium hydride (11.4 mL, $17.0 \mathrm{mmol}, 3.0$ equiv) in toluene $(1.5 \mathrm{M})$ via syringe. The reaction mixture was slowly warmed to room temperature and stirred for 1 hour. The reaction was quenched with saturated aqueous $\mathrm{NH}_{4} \mathrm{Cl}$ solution. The organic layer was separated and the aqueous layer was extracted with $\mathrm{CH}_{2} \mathrm{Cl}_{2}(2 \times 50$ $\mathrm{mL}$ ). The combined organic phase was washed with brine, dried over $\mathrm{Na}_{2} \mathrm{SO}_{4}$, and concentrated in vacuo. The residue was purified by flash column chromatography $\left(\mathrm{SiO}_{2}\right.$, ethyl acetate/hexane) gave paeoveitol D (2) (1.1 g, 88\%) as a white solid: m.p. $=157-159^{\circ} \mathrm{C}$; ${ }^{1} \mathrm{H}$ NMR (600 MHz, MeOH-d $d^{4}$ ) $\delta 7.53$ (s, 1H), 7.16 (s, 1H), 6.99 (s, 1H), 4.88 (s, 2H), 4.66 (s, 2H), 2.28 (s, 3H); ${ }^{13} \mathrm{C}$ NMR (151 MHz, MeOH-d $\left.{ }^{4}\right) \delta$ 152.6, 151.6, 143.4, 126.6, 124.1, 121.6, 113.3, 104.9, 55.8, 17.1; IR (thin film, $\mathrm{cm}^{-1}$ ) 3500, 3200, 2960, 2860, 1480,1400, 1180, 995, 870, 810 ; HRMS (ESI) calcd. for $\left[\mathrm{C}_{10} \mathrm{H}_{10} \mathrm{O}_{3} \mathrm{Na}\right]^{+}(\mathrm{M}+\mathrm{Na})^{+}: \mathrm{m} / z$ 201.0522, found 201.0525 . 
Diol 3. To a solution of ketone $\mathbf{1 3}^{[1]}(800 \mathrm{mg}, 4.8 \mathrm{mmol}, 1.0 \mathrm{equiv})$ in
3.0 equiv) in $\mathrm{THF}(1.0 \mathrm{M})$ via syringe. The reaction mixture was slowly warmed to room temperature and stirred for 1 hour at same temperature. The reaction was quenched with water $(5 \mathrm{~mL})$. The organic layer was separated and the aqueous layer was extracted with $\mathrm{Et}_{2} \mathrm{O}(50 \mathrm{~mL})$. The combined organic phase was washed with brine, dried over $\mathrm{Na}_{2} \mathrm{SO}_{4}$, and concentrated in vacuo. The residue was purified by flash column chromatography ( $\mathrm{SiO}_{2}$, ethyl acetate/hexane) gave diol 3 (694 mg, 86\%) as a colorless oil: ${ }^{1} \mathrm{H}$ NMR (600 MHz, CDCl $) \delta 7.65(\mathrm{~s}, 1 \mathrm{H}), 6.63-6.54(\mathrm{~m}, 1 \mathrm{H}), 6.43(\mathrm{~s}, 1 \mathrm{H}), 5.94(\mathrm{~s}, 1 \mathrm{H}), 4.91$ $(\mathrm{q}, J=6.6 \mathrm{~Hz}, 1 \mathrm{H}), 3.71(\mathrm{~s}, 1 \mathrm{H}), 2.20-2.11(\mathrm{~m}, 3 \mathrm{H}), 1.49(\mathrm{~d}, J=6.6 \mathrm{~Hz}, 3 \mathrm{H}) ;{ }^{13} \mathrm{C}$ NMR $(151$ $\mathrm{MHz}_{\mathrm{CDCl}}$ ) $\delta 148.2,147.2,127.2,124.4,118.8,113.0,70.2,23.2,15.6$; IR (thin film, $\mathrm{cm}^{-1}$ ) $3382,2978,2928,1634,1506,1420,1190 ; \mathrm{HRMS}(\mathrm{ESI})$ calcd. for $\left[\mathrm{C}_{9} \mathrm{H}_{12} \mathrm{O}_{3} \mathrm{Na}\right]^{+}(\mathrm{M}+\mathrm{Na})^{+}: m / z$ 191.0679, found 191.0684 .

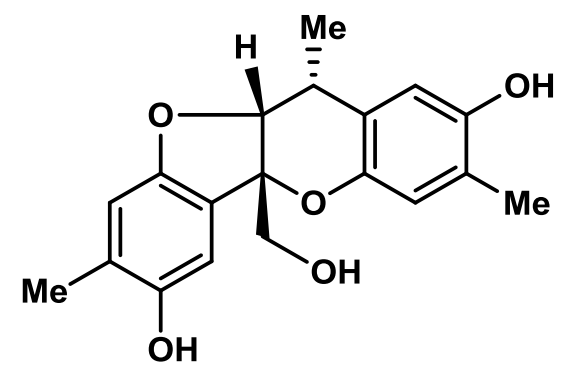

Paeoveitol (1). A solution of 2 (1.1 g, $6.2 \mathrm{mmol}, 1.0$ equiv) and diol 3 (1.25 g, $7.4 \mathrm{mmol}, 1.2$ equiv) in $\mathrm{CH}_{2} \mathrm{Cl}_{2}(40 \mathrm{~mL})$ was treated with anhydrous $\mathrm{ZnCl}_{2}(85.0 \mathrm{mg}, 0.62 \mathrm{mmol}, 0.1$ equiv) at room temperature. After 12 hours, the reaction mixture was quenched with water $(2 \mathrm{~mL})$ and extracted with $\mathrm{CH}_{2} \mathrm{Cl}_{2}(2 \times 50 \mathrm{~mL})$. The organic phase was washed with brine, dried over $\mathrm{Na}_{2} \mathrm{SO}_{4}$, and concentrated in vacuo. The residue was purified by flash column chromatography $\mathrm{SiO}_{2}$, ethyl acetate/hexane) gave paeoveitol (1) (1.34 g, 66\%) as a White foam: m.p. $=151-15{ }^{\circ} \mathrm{C}$; ${ }^{1} \mathrm{H}$ NMR (600 MHz, DMSO-d $) \delta 8.75(\mathrm{~s}, 1 \mathrm{H}), 8.70(\mathrm{~s}, 1 \mathrm{H}), 6.68(\mathrm{~s}, 1 \mathrm{H}), 6.59-6.53(\mathrm{~m}, 1 \mathrm{H})$, $6.32(\mathrm{~s}, 1 \mathrm{H}), 6.30(\mathrm{~s}, 1 \mathrm{H}), 5.26-5.18(\mathrm{~m}, 1 \mathrm{H}), 4.97(\mathrm{~d}, J=3.0 \mathrm{~Hz}, 1 \mathrm{H}), 3.95(\mathrm{dd}, J=11.5,5.5$ Hz, 1H), 3.77 (dd, $J=11.5,6.7 \mathrm{~Hz}, 1 \mathrm{H}), 3.34$ (d, $J=1.7 \mathrm{~Hz}, 3 \mathrm{H}), 2.99-2.88(\mathrm{~m}, 1 \mathrm{H}), 2.50$ (p, $J$ $=1.8 \mathrm{~Hz}, 3 \mathrm{H}), 1.97(\mathrm{~s}, 3 \mathrm{H}), 1.93(\mathrm{~s}, 3 \mathrm{H}), 1.41(\mathrm{~d}, J=7.0 \mathrm{~Hz}, 3 \mathrm{H}) ;{ }^{13} \mathrm{C}$ NMR (151 MHz, DMSO$\left.d^{6}\right) \delta 152.8,150.1,149.0,146.0,126.6,126.4,124.7,121.5,118.9,112.1,110.6,110.0,88.2$, 87.9, 65.0, 31.6, 16.4, 15.6, 13.2; IR (thin film, $\mathrm{cm}^{-1}$ ) 3680, 2920, 2850, 1630, 1480, 1179; HRMS (ESI) calcd. for $\left[\mathrm{C}_{19} \mathrm{H}_{20} \mathrm{O}_{5} \mathrm{Na}\right]^{+}(\mathrm{M}+\mathrm{Na})^{+}: \mathrm{m} / z 351.1203$ found 351.1208 . 


\section{X-ray crystallography 9 and 1}

\subsection{X-ray crystal structure determination of 9}

SI Table 1. Crystal data and structure refinement for 9.

Identification code

Empirical formula

Formula weight

Temperature

Wavelength

Crystal system

Space group

Unit cell dimensions

Volume

$\mathrm{Z}$

Density (calculated)

Absorption coefficient

$\mathrm{F}(000)$

Crystal size

Theta range for data collection

Index ranges

Reflections collected

Independent reflections

Completeness to theta $=25.242^{\circ}$

Refinement method

Data / restraints / parameters

Goodness-of-fit on $\mathrm{F}^{2}$

Final R indices [I $>2 \operatorname{sigma}(\mathrm{I})]$

$\mathrm{R}$ indices (all data)

Extinction coefficient

Largest diff. peak and hole xl-176-2

$\mathrm{C} 24 \mathrm{H} 22 \mathrm{O} 5 \mathrm{~S}$

422.47

153(2) K

$0.71073 \AA$

Triclinic

P-1

$\mathrm{a}=10.0910(3) \AA$

$\alpha=67.4450(10)^{\circ}$.

$\mathrm{b}=10.4061(3) \AA$

$\beta=72.9880(10)^{\circ}$.

$\mathrm{c}=11.4027(3) \AA$ $\gamma=70.3200(10)^{\circ}$.

1022.65(5) $\AA^{3}$

2

$1.372 \mathrm{Mg} / \mathrm{m}^{3}$

$0.192 \mathrm{~mm}^{-1}$

444

$0.3 \times 0.1 \times 0.1 \mathrm{~mm}^{3}$

2.191 to $26.458^{\circ}$.

$-12<=\mathrm{h}<=12,-13<=\mathrm{k}<=13,-14<=1<=14$

32260

$4167[\mathrm{R}(\mathrm{int})=0.0217]$

$98.8 \%$

Full-matrix least-squares on $\mathrm{F}^{2}$

4167 / 0 / 271

1.047

$\mathrm{R} 1=0.0373, \mathrm{wR} 2=0.0991$

$\mathrm{R} 1=0.0391, \mathrm{wR} 2=0.1009$

$\mathrm{n} / \mathrm{a}$

0.575 and -0.479 e. $\AA^{-3}$ 
SI Table 2. Atomic coordinates (x 104) and equivalent isotropic displacement parameters $\left(\AA^{2} \times 10^{3}\right)$ for $x l-176-2 . ~ U(e q)$ is defined as one third of the trace of the orthogonalized $U^{i j}$ tensor.

\begin{tabular}{|c|c|c|c|c|}
\hline & $\mathrm{x}$ & $y$ & $\mathrm{z}$ & $\mathrm{U}(\mathrm{eq})$ \\
\hline$S(1)$ & 2739(1) & $6326(1)$ & $3960(1)$ & $20(1)$ \\
\hline $\mathrm{O}(3)$ & $3235(1)$ & $6764(1)$ & $2439(1)$ & $22(1)$ \\
\hline $\mathrm{O}(4)$ & 4803(1) & $6748(1)$ & $-1436(1)$ & $24(1)$ \\
\hline $\mathrm{O}(5)$ & $2128(1)$ & $8706(1)$ & $280(1)$ & $19(1)$ \\
\hline $\mathrm{O}(1)$ & 2604(1) & 4881(1) & $4450(1)$ & $28(1)$ \\
\hline $\mathrm{O}(2)$ & 3723(1) & 6693(1) & $4374(1)$ & $29(1)$ \\
\hline$C(7)$ & $2545(2)$ & $6360(2)$ & $1727(1)$ & $21(1)$ \\
\hline $\mathrm{C}(8)$ & 1028(2) & $7487(2)$ & $4172(1)$ & $20(1)$ \\
\hline $\mathrm{C}(9)$ & $2608(2)$ & $9770(1)$ & $-786(1)$ & $18(1)$ \\
\hline$C(10)$ & $5047(2)$ & $8332(2)$ & $-433(1)$ & $22(1)$ \\
\hline $\mathrm{C}(11)$ & $4522(2)$ & $7032(2)$ & $-214(1)$ & $20(1)$ \\
\hline $\mathrm{C}(12)$ & $-1441(2)$ & $7860(2)$ & 5201(2) & $27(1)$ \\
\hline$C(13)$ & 2063(2) & $12074(2)$ & $-2372(2)$ & $26(1)$ \\
\hline$C(14)$ & $816(2)$ & $8954(2)$ & $3505(2)$ & $27(1)$ \\
\hline$C(15)$ & $3515(2)$ & 11990(2) & $-2808(2)$ & $28(1)$ \\
\hline$C(16)$ & $4074(2)$ & $9658(2)$ & $-1191(1)$ & 19(1) \\
\hline $\mathrm{C}(17)$ & 2888(2) & 7239(1) & $320(1)$ & $18(1)$ \\
\hline $\mathrm{C}(18)$ & $-93(2)$ & 6932(2) & $5025(1)$ & $24(1)$ \\
\hline $\mathrm{C}(19)$ & 1594(2) & 10953(2) & $-1360(1)$ & $22(1)$ \\
\hline $\mathrm{C}(20)$ & $-536(2)$ & $9856(2)$ & $3690(2)$ & $29(1)$ \\
\hline $\mathrm{C}(21)$ & $2429(2)$ & $6759(2)$ & $-555(1)$ & $20(1)$ \\
\hline $\mathrm{C}(22)$ & $-1686(2)$ & 9321(2) & 4529(2) & $26(1)$ \\
\hline $\mathrm{C}(23)$ & 4512(2) & 10782(2) & $-2223(2)$ & $24(1)$ \\
\hline $\mathrm{C}(24)$ & $3587(2)$ & $6508(2)$ & $-1528(1)$ & $22(1)$ \\
\hline$C(25)$ & $6645(2)$ & $8112(2)$ & $-997(2)$ & $32(1)$ \\
\hline$C(26)$ & 1120(2) & $6576(2)$ & $-524(2)$ & $26(1)$ \\
\hline $\mathrm{C}(27)$ & $3506(2)$ & $6045(2)$ & $-2486(2)$ & $27(1)$ \\
\hline $\mathrm{C}(28)$ & 2196(2) & $5834(2)$ & $-2428(2)$ & $30(1)$ \\
\hline $\mathrm{C}(29)$ & 1013(2) & 6102(2) & $-1472(2)$ & $31(1)$ \\
\hline$C(30)$ & $-3168(2)$ & $10303(2)$ & $4655(2)$ & $37(1)$ \\
\hline
\end{tabular}


SI Table 3. Bond lengths $[\AA ̊]$ and angles $\left[^{\circ}\right]$ for xl-176-2.

\begin{tabular}{|c|c|}
\hline $\mathrm{S}(1)-\mathrm{O}(2)$ & $1.4226(11)$ \\
\hline $\mathrm{S}(1)-\mathrm{O}(1)$ & $1.4283(11)$ \\
\hline $\mathrm{S}(1)-\mathrm{O}(3)$ & $1.5821(10)$ \\
\hline $\mathrm{S}(1)-\mathrm{C}(8)$ & $1.7535(15)$ \\
\hline $\mathrm{O}(3)-\mathrm{C}(7)$ & $1.4530(17)$ \\
\hline $\mathrm{O}(4)-\mathrm{C}(24)$ & $1.3722(18)$ \\
\hline $\mathrm{O}(4)-\mathrm{C}(11)$ & $1.4618(16)$ \\
\hline $\mathrm{O}(5)-\mathrm{C}(9)$ & $1.3866(16)$ \\
\hline $\mathrm{O}(5)-\mathrm{C}(17)$ & $1.4481(16)$ \\
\hline $\mathrm{C}(7)-\mathrm{C}(17)$ & $1.5147(19)$ \\
\hline $\mathrm{C}(8)-\mathrm{C}(18)$ & $1.387(2)$ \\
\hline $\mathrm{C}(8)-\mathrm{C}(14)$ & $1.392(2)$ \\
\hline $\mathrm{C}(9)-\mathrm{C}(19)$ & $1.384(2)$ \\
\hline $\mathrm{C}(9)-\mathrm{C}(16)$ & $1.393(2)$ \\
\hline$C(10)-C(16)$ & $1.511(2)$ \\
\hline $\mathrm{C}(10)-\mathrm{C}(11)$ & $1.523(2)$ \\
\hline$C(10)-C(25)$ & $1.525(2)$ \\
\hline $\mathrm{C}(11)-\mathrm{C}(17)$ & $1.5524(19)$ \\
\hline$C(12)-C(22)$ & $1.387(2)$ \\
\hline $\mathrm{C}(12)-\mathrm{C}(18)$ & $1.388(2)$ \\
\hline $\mathrm{C}(13)-\mathrm{C}(15)$ & $1.386(2)$ \\
\hline$C(13)-C(19)$ & $1.391(2)$ \\
\hline$C(14)-C(20)$ & $1.381(2)$ \\
\hline $\mathrm{C}(15)-\mathrm{C}(23)$ & $1.391(2)$ \\
\hline$C(16)-C(23)$ & $1.391(2)$ \\
\hline $\mathrm{C}(17)-\mathrm{C}(21)$ & $1.5050(19)$ \\
\hline$C(20)-C(22)$ & $1.397(2)$ \\
\hline$C(21)-C(26)$ & $1.386(2)$ \\
\hline $\mathrm{C}(21)-\mathrm{C}(24)$ & $1.387(2)$ \\
\hline $\mathrm{C}(22)-\mathrm{C}(30)$ & $1.503(2)$ \\
\hline $\mathrm{C}(24)-\mathrm{C}(27)$ & $1.384(2)$ \\
\hline$C(26)-C(29)$ & $1.392(2)$ \\
\hline$C(27)-C(28)$ & $1.390(2)$ \\
\hline
\end{tabular}




\begin{tabular}{|c|c|}
\hline$C(28)-C(29)$ & $1.392(2)$ \\
\hline $\mathrm{O}(2)-\mathrm{S}(1)-\mathrm{O}(1)$ & $119.87(7)$ \\
\hline $\mathrm{O}(2)-\mathrm{S}(1)-\mathrm{O}(3)$ & $103.80(6)$ \\
\hline $\mathrm{O}(1)-\mathrm{S}(1)-\mathrm{O}(3)$ & $109.36(6)$ \\
\hline $\mathrm{O}(2)-\mathrm{S}(1)-\mathrm{C}(8)$ & $110.42(7)$ \\
\hline $\mathrm{O}(1)-\mathrm{S}(1)-\mathrm{C}(8)$ & $108.64(7)$ \\
\hline $\mathrm{O}(3)-\mathrm{S}(1)-\mathrm{C}(8)$ & $103.46(6)$ \\
\hline $\mathrm{C}(7)-\mathrm{O}(3)-\mathrm{S}(1)$ & $117.52(9)$ \\
\hline $\mathrm{C}(24)-\mathrm{O}(4)-\mathrm{C}(11)$ & $107.61(11)$ \\
\hline $\mathrm{C}(9)-\mathrm{O}(5)-\mathrm{C}(17)$ & $115.73(10)$ \\
\hline $\mathrm{O}(3)-\mathrm{C}(7)-\mathrm{C}(17)$ & $106.71(11)$ \\
\hline$C(18)-C(8)-C(14)$ & $120.87(14)$ \\
\hline $\mathrm{C}(18)-\mathrm{C}(8)-\mathrm{S}(1)$ & $119.35(12)$ \\
\hline $\mathrm{C}(14)-\mathrm{C}(8)-\mathrm{S}(1)$ & $119.76(11)$ \\
\hline $\mathrm{C}(19)-\mathrm{C}(9)-\mathrm{O}(5)$ & $118.04(12)$ \\
\hline$C(19)-C(9)-C(16)$ & $122.45(13)$ \\
\hline $\mathrm{O}(5)-\mathrm{C}(9)-\mathrm{C}(16)$ & $119.39(12)$ \\
\hline$C(16)-C(10)-C(11)$ & $108.22(12)$ \\
\hline$C(16)-C(10)-C(25)$ & $115.86(13)$ \\
\hline $\mathrm{C}(11)-\mathrm{C}(10)-\mathrm{C}(25)$ & $112.01(13)$ \\
\hline $\mathrm{O}(4)-\mathrm{C}(11)-\mathrm{C}(10)$ & $109.84(11)$ \\
\hline $\mathrm{O}(4)-\mathrm{C}(11)-\mathrm{C}(17)$ & $106.81(11)$ \\
\hline $\mathrm{C}(10)-\mathrm{C}(11)-\mathrm{C}(17)$ & $113.35(11)$ \\
\hline $\mathrm{C}(22)-\mathrm{C}(12)-\mathrm{C}(18)$ & $121.21(14)$ \\
\hline $\mathrm{C}(15)-\mathrm{C}(13)-\mathrm{C}(19)$ & $120.50(14)$ \\
\hline $\mathrm{C}(20)-\mathrm{C}(14)-\mathrm{C}(8)$ & $119.09(14)$ \\
\hline $\mathrm{C}(13)-\mathrm{C}(15)-\mathrm{C}(23)$ & $119.87(14)$ \\
\hline $\mathrm{C}(23)-\mathrm{C}(16)-\mathrm{C}(9)$ & $117.78(13)$ \\
\hline $\mathrm{C}(23)-\mathrm{C}(16)-\mathrm{C}(10)$ & $125.85(13)$ \\
\hline $\mathrm{C}(9)-\mathrm{C}(16)-\mathrm{C}(10)$ & $116.35(12)$ \\
\hline $\mathrm{O}(5)-\mathrm{C}(17)-\mathrm{C}(21)$ & $112.01(11)$ \\
\hline $\mathrm{O}(5)-\mathrm{C}(17)-\mathrm{C}(7)$ & $104.41(11)$ \\
\hline $\mathrm{C}(21)-\mathrm{C}(17)-\mathrm{C}(7)$ & $112.04(11)$ \\
\hline $\mathrm{O}(5)-\mathrm{C}(17)-\mathrm{C}(11)$ & $113.59(11)$ \\
\hline
\end{tabular}




$\begin{array}{ll}\mathrm{C}(21)-\mathrm{C}(17)-\mathrm{C}(11) & 102.16(11) \\ \mathrm{C}(7)-\mathrm{C}(17)-\mathrm{C}(11) & 112.92(11) \\ \mathrm{C}(8)-\mathrm{C}(18)-\mathrm{C}(12) & 119.06(14) \\ \mathrm{C}(9)-\mathrm{C}(19)-\mathrm{C}(13) & 118.50(14) \\ \mathrm{C}(14)-\mathrm{C}(20)-\mathrm{C}(22) & 121.14(15) \\ \mathrm{C}(26)-\mathrm{C}(21)-\mathrm{C}(24) & 120.37(14) \\ \mathrm{C}(26)-\mathrm{C}(21)-\mathrm{C}(17) & 131.10(13) \\ \mathrm{C}(24)-\mathrm{C}(21)-\mathrm{C}(17) & 108.52(13) \\ \mathrm{C}(12)-\mathrm{C}(22)-\mathrm{C}(20) & 118.61(15) \\ \mathrm{C}(12)-\mathrm{C}(22)-\mathrm{C}(30) & 120.96(15) \\ \mathrm{C}(20)-\mathrm{C}(22)-\mathrm{C}(30) & 120.39(15) \\ \mathrm{C}(15)-\mathrm{C}(23)-\mathrm{C}(16) & 120.89(14) \\ \mathrm{O}(4)-\mathrm{C}(24)-\mathrm{C}(27) & 123.94(14) \\ \mathrm{O}(4)-\mathrm{C}(24)-\mathrm{C}(21) & 113.64(13) \\ \mathrm{C}(27)-\mathrm{C}(24)-\mathrm{C}(21) & 122.42(15) \\ \mathrm{C}(21)-\mathrm{C}(26)-\mathrm{C}(29) & 118.33(15) \\ \mathrm{C}(24)-\mathrm{C}(27)-\mathrm{C}(28) & 116.61(15) \\ \mathrm{C}(27)-\mathrm{C}(28)-\mathrm{C}(29) & 122.00(15) \\ \mathrm{C}(26)-\mathrm{C}(29)-\mathrm{C}(28) & 120.24(16)\end{array}$

Symmetry transformations used to generate equivalent atoms: 
SI Table 4. Anisotropic displacement parameters $\left(\AA^{2} \times 10^{3}\right)$ for $x 1-176-2$. The anisotropic displacement factor exponent takes the form: $-2 \pi^{2}\left[h^{2} a^{* 2} U^{11}+\ldots+2 h k a^{*} b^{*} U^{12}\right]$

\begin{tabular}{|c|c|c|c|c|c|c|}
\hline & $\mathrm{U}^{11}$ & $\mathrm{U}^{22}$ & $\mathrm{U}^{33}$ & $\mathrm{U}^{23}$ & $\mathrm{U}^{13}$ & $\mathrm{U}^{12}$ \\
\hline$S(1)$ & $23(1)$ & $22(1)$ & $14(1)$ & $-4(1)$ & $-2(1)$ & $-7(1)$ \\
\hline $\mathrm{O}(3)$ & $24(1)$ & $26(1)$ & $14(1)$ & $-6(1)$ & $-1(1)$ & $-9(1)$ \\
\hline $\mathrm{O}(4)$ & $23(1)$ & $28(1)$ & $20(1)$ & $-12(1)$ & $-1(1)$ & $-3(1)$ \\
\hline $\mathrm{O}(5)$ & $20(1)$ & $16(1)$ & 19(1) & $-6(1)$ & $1(1)$ & $-3(1)$ \\
\hline $\mathrm{O}(1)$ & $33(1)$ & $21(1)$ & $23(1)$ & $-1(1)$ & $-4(1)$ & $-6(1)$ \\
\hline $\mathrm{O}(2)$ & $28(1)$ & $42(1)$ & $22(1)$ & $-10(1)$ & $-5(1)$ & $-11(1)$ \\
\hline$C(7)$ & $26(1)$ & $21(1)$ & $16(1)$ & $-6(1)$ & $-3(1)$ & $-8(1)$ \\
\hline $\mathrm{C}(8)$ & $23(1)$ & $22(1)$ & $17(1)$ & $-7(1)$ & $-2(1)$ & $-7(1)$ \\
\hline $\mathrm{C}(9)$ & $21(1)$ & $17(1)$ & $16(1)$ & $-7(1)$ & $-2(1)$ & $-7(1)$ \\
\hline$C(10)$ & $19(1)$ & $27(1)$ & $19(1)$ & $-8(1)$ & $-4(1)$ & $-5(1)$ \\
\hline $\mathrm{C}(11)$ & $21(1)$ & $21(1)$ & $16(1)$ & $-7(1)$ & $-3(1)$ & $-1(1)$ \\
\hline $\mathrm{C}(12)$ & $26(1)$ & $33(1)$ & $26(1)$ & $-13(1)$ & $3(1)$ & $-13(1)$ \\
\hline$C(13)$ & $31(1)$ & $19(1)$ & $27(1)$ & $-4(1)$ & $-10(1)$ & $-4(1)$ \\
\hline$C(14)$ & $28(1)$ & $24(1)$ & $26(1)$ & $-5(1)$ & $0(1)$ & $-12(1)$ \\
\hline$C(15)$ & $34(1)$ & $24(1)$ & $23(1)$ & $-3(1)$ & $-3(1)$ & $-13(1)$ \\
\hline$C(16)$ & $21(1)$ & $21(1)$ & $18(1)$ & $-9(1)$ & $-3(1)$ & $-6(1)$ \\
\hline $\mathrm{C}(17)$ & $21(1)$ & $15(1)$ & $17(1)$ & $-5(1)$ & $-2(1)$ & $-3(1)$ \\
\hline$C(18)$ & $29(1)$ & $24(1)$ & $20(1)$ & $-6(1)$ & $0(1)$ & $-12(1)$ \\
\hline$C(19)$ & $21(1)$ & $20(1)$ & $24(1)$ & $-8(1)$ & $-4(1)$ & $-4(1)$ \\
\hline$C(20)$ & $34(1)$ & 21(1) & $31(1)$ & $-8(1)$ & $-4(1)$ & $-7(1)$ \\
\hline$C(21)$ & $27(1)$ & $16(1)$ & $16(1)$ & $-4(1)$ & $-4(1)$ & $-4(1)$ \\
\hline$C(22)$ & $27(1)$ & $30(1)$ & $26(1)$ & $-16(1)$ & $-3(1)$ & $-7(1)$ \\
\hline$C(23)$ & $24(1)$ & $28(1)$ & $22(1)$ & $-9(1)$ & $0(1)$ & $-12(1)$ \\
\hline$C(24)$ & $27(1)$ & $16(1)$ & $18(1)$ & $-3(1)$ & $-5(1)$ & $-2(1)$ \\
\hline$C(25)$ & $20(1)$ & $41(1)$ & $34(1)$ & $-12(1)$ & $-4(1)$ & $-6(1)$ \\
\hline$C(26)$ & $30(1)$ & $26(1)$ & $22(1)$ & $-6(1)$ & $-2(1)$ & $-10(1)$ \\
\hline $\mathrm{C}(27)$ & $37(1)$ & $23(1)$ & $18(1)$ & $-8(1)$ & $-5(1)$ & $-2(1)$ \\
\hline$C(28)$ & $48(1)$ & $26(1)$ & $22(1)$ & $-8(1)$ & $-11(1)$ & $-10(1)$ \\
\hline$C(29)$ & $38(1)$ & $31(1)$ & $28(1)$ & $-6(1)$ & $-9(1)$ & $-15(1)$ \\
\hline$C(30)$ & $30(1)$ & $38(1)$ & $44(1)$ & $-20(1)$ & $-4(1)$ & $-3(1)$ \\
\hline
\end{tabular}


SI Table 5. Hydrogen coordinates ( x 10 $0^{4}$ ) and isotropic displacement parameters $\left(\AA^{2} \times 10^{3}\right)$ for xl-176-2.

\begin{tabular}{|c|c|c|c|c|}
\hline & $\mathrm{x}$ & $\mathrm{y}$ & $\mathrm{z}$ & $\mathrm{U}(\mathrm{eq})$ \\
\hline $\mathrm{H}(7 \mathrm{~A})$ & 1496 & 6569 & 2045 & 25 \\
\hline $\mathrm{H}(7 \mathrm{~B})$ & 2915 & 5319 & 1834 & 25 \\
\hline $\mathrm{H}(10 \mathrm{~A})$ & 4908 & 8430 & 436 & 26 \\
\hline $\mathrm{H}(11 \mathrm{~A})$ & 5051 & 6174 & 408 & 24 \\
\hline $\mathrm{H}(12 \mathrm{~A})$ & -2209 & 7487 & 5792 & 33 \\
\hline $\mathrm{H}(13 \mathrm{~A})$ & 1383 & 12903 & -2767 & 32 \\
\hline $\mathrm{H}(14 \mathrm{~A})$ & 1591 & 9328 & 2931 & 32 \\
\hline $\mathrm{H}(15 \mathrm{~A})$ & 3828 & 12755 & -3506 & 33 \\
\hline $\mathrm{H}(18 \mathrm{~A})$ & 60 & 5931 & 5483 & 29 \\
\hline $\mathrm{H}(19 \mathrm{~A})$ & 601 & 10999 & -1069 & 26 \\
\hline $\mathrm{H}(20 \mathrm{~A})$ & -685 & 10858 & 3238 & 35 \\
\hline $\mathrm{H}(23 \mathrm{~A})$ & 5506 & 10725 & -2531 & 29 \\
\hline $\mathrm{H}(25 \mathrm{~A})$ & 7179 & 7228 & -438 & 49 \\
\hline $\mathrm{H}(25 \mathrm{~B})$ & 6827 & 8033 & -1863 & 49 \\
\hline $\mathrm{H}(25 \mathrm{C})$ & 6957 & 8934 & -1054 & 49 \\
\hline $\mathrm{H}(26 \mathrm{~A})$ & 316 & 6769 & 127 & 31 \\
\hline $\mathrm{H}(27 \mathrm{~A})$ & 4305 & 5880 & -3150 & 33 \\
\hline $\mathrm{H}(28 \mathrm{~A})$ & 2106 & 5498 & -3059 & 36 \\
\hline $\mathrm{H}(29 \mathrm{~A})$ & 129 & 5961 & -1468 & 37 \\
\hline $\mathrm{H}(30 \mathrm{~A})$ & -3134 & 11290 & 4114 & 56 \\
\hline $\mathrm{H}(30 \mathrm{~B})$ & -3802 & 9990 & 4373 & 56 \\
\hline $\mathrm{H}(30 \mathrm{C})$ & -3533 & 10271 & 5560 & 56 \\
\hline
\end{tabular}




\subsection{X-ray crystal structure determination of 1}

SI Table 6. Crystal data and structure refinement for $\mathbf{1}$.

Identification code

Empirical formula

Formula weight

Temperature

Wavelength

Crystal system

Space group

Unit cell dimensions

Volume

Z

Density (calculated)

Absorption coefficient

$\mathrm{F}(000)$

Crystal size

Theta range for data collection

Index ranges

Reflections collected

Independent reflections

Completeness to theta $=26.000^{\circ}$

Absorption correction

Max. and min. transmission

Refinement method

Data / restraints / parameters

Goodness-of-fit on $\mathrm{F}^{2}$

Final $\mathrm{R}$ indices [I $>2 \operatorname{sigma}(\mathrm{I})]$

$\mathrm{R}$ indices (all data)

Largest diff. peak and hole xl-276-1

C19 H20 O5

328.35

$153.0 \mathrm{~K}$

$0.71073 \AA$

Monoclinic

$C 2 / c$

$a=19.3064(9) \AA \quad \alpha=90^{\circ}$.

$\mathrm{b}=8.9818(4) \AA$

$\beta=95.154(2)^{\circ}$.

$\mathrm{c}=18.1833(8) \AA$

$\gamma=90^{\circ}$.

3140.3(2) $\AA^{3}$

8

$1.389 \mathrm{Mg} / \mathrm{m}^{3}$

$0.100 \mathrm{~mm}^{-1}$

1392

$0.5 \times 0.4 \times 0.3 \mathrm{~mm}^{3}$

2.118 to $27.553^{\circ}$.

$-25<=\mathrm{h}<=25,-11<=\mathrm{k}<=11,-23<=\mathrm{l}<=23$

40040

$3621[\mathrm{R}(\mathrm{int})=0.0563]$

$99.9 \%$

Semi-empirical from equivalents

0.7456 and 0.6954

Full-matrix least-squares on $\mathrm{F}^{2}$

$3621 / 0 / 223$

1.040

$\mathrm{R} 1=0.0467, \mathrm{wR} 2=0.1186$

$\mathrm{R} 1=0.0598, \mathrm{wR} 2=0.1261$

0.570 and -0.287 e. $\AA^{-3}$ 
SI Table 7. Atomic coordinates (x $\left.10^{4}\right)$ and equivalent isotropic displacement parameters $\left(\AA^{2} \times 10^{3}\right)$ for $\mathrm{xl}-276-1 . \mathrm{U}(\mathrm{eq})$ is defined as one third of the trace of the orthogonalized $\mathrm{U}^{\mathrm{ij}}$ tensor.

\begin{tabular}{|c|c|c|c|c|}
\hline & $\mathrm{x}$ & $\mathrm{y}$ & $\mathrm{z}$ & $\mathrm{U}(\mathrm{eq})$ \\
\hline $\mathrm{O}(2)$ & $6978(1)$ & $2780(1)$ & 4081(1) & $22(1)$ \\
\hline $\mathrm{O}(1)$ & $5328(1)$ & 1511(1) & 4201(1) & $22(1)$ \\
\hline $\mathrm{O}(4)$ & $6292(1)$ & $-933(1)$ & $1728(1)$ & $27(1)$ \\
\hline $\mathrm{O}(5)$ & $4915(1)$ & $7446(1)$ & $3523(1)$ & $33(1)$ \\
\hline $\mathrm{O}(3)$ & $5887(1)$ & $-236(2)$ & $5417(1)$ & $33(1)$ \\
\hline$C(6)$ & $6325(1)$ & $879(2)$ & $3517(1)$ & 19(1) \\
\hline $\mathrm{C}(3)$ & $6990(1)$ & $1140(2)$ & $2225(1)$ & $20(1)$ \\
\hline$C(7)$ & $6842(1)$ & 1922(2) & $3457(1)$ & 19(1) \\
\hline $\mathrm{C}(4)$ & $6468(1)$ & $68(2)$ & $2292(1)$ & $20(1)$ \\
\hline$C(5)$ & $6130(1)$ & $-67(2)$ & 2931(1) & $20(1)$ \\
\hline $\mathrm{C}(2)$ & $7175(1)$ & $2080(2)$ & $2822(1)$ & $21(1)$ \\
\hline$C(15)$ & $4695(1)$ & $3379(2)$ & $3476(1)$ & $22(1)$ \\
\hline$C(14)$ & $5222(1)$ & $3002(2)$ & $4005(1)$ & 19(1) \\
\hline$C(16)$ & $4559(1)$ & $4877(2)$ & $3305(1)$ & $22(1)$ \\
\hline$C(13)$ & $5637(1)$ & $4068(2)$ & 4381(1) & $21(1)$ \\
\hline $\mathrm{C}(9)$ & $6054(1)$ & $1029(2)$ & $4264(1)$ & $20(1)$ \\
\hline $\mathrm{C}(17)$ & 4984(1) & $5944(2)$ & $3670(1)$ & $23(1)$ \\
\hline$C(10)$ & $6554(1)$ & $2213(2)$ & $4643(1)$ & $20(1)$ \\
\hline $\mathrm{C}(11)$ & $6177(1)$ & $3512(2)$ & $4970(1)$ & $22(1)$ \\
\hline$C(18)$ & $5517(1)$ & $5548(2)$ & $4200(1)$ & $24(1)$ \\
\hline $\mathrm{C}(1)$ & 7343(1) & $1277(2)$ & $1523(1)$ & $30(1)$ \\
\hline$C(12)$ & $6675(1)$ & $4698(2)$ & $5302(1)$ & $28(1)$ \\
\hline $\mathrm{C}(8)$ & $6052(1)$ & $-436(2)$ & $4682(1)$ & $25(1)$ \\
\hline$C(19)$ & $3970(1)$ & $5303(2)$ & $2744(1)$ & $31(1)$ \\
\hline
\end{tabular}


SI Table 8. Bond lengths $[\AA ̊]$ and angles $\left[{ }^{\circ}\right]$ for xl-276-1.

\begin{tabular}{|c|c|}
\hline $\mathrm{O}(2)-\mathrm{C}(7)$ & $1.3775(18)$ \\
\hline $\mathrm{O}(2)-\mathrm{C}(10)$ & $1.4580(18)$ \\
\hline $\mathrm{O}(1)-\mathrm{C}(14)$ & $1.3960(18)$ \\
\hline $\mathrm{O}(1)-\mathrm{C}(9)$ & $1.4613(18)$ \\
\hline $\mathrm{O}(4)-\mathrm{H}(4)$ & 0.8400 \\
\hline $\mathrm{O}(4)-\mathrm{C}(4)$ & $1.3836(18)$ \\
\hline $\mathrm{O}(5)-\mathrm{H}(5)$ & 0.8400 \\
\hline $\mathrm{O}(5)-\mathrm{C}(17)$ & $1.3795(19)$ \\
\hline $\mathrm{O}(3)-\mathrm{H}(3)$ & 0.8400 \\
\hline $\mathrm{O}(3)-\mathrm{C}(8)$ & $1.412(2)$ \\
\hline $\mathrm{C}(6)-\mathrm{C}(7)$ & $1.380(2)$ \\
\hline $\mathrm{C}(6)-\mathrm{C}(5)$ & $1.388(2)$ \\
\hline $\mathrm{C}(6)-\mathrm{C}(9)$ & $1.504(2)$ \\
\hline $\mathrm{C}(3)-\mathrm{C}(4)$ & $1.406(2)$ \\
\hline $\mathrm{C}(3)-\mathrm{C}(2)$ & $1.396(2)$ \\
\hline $\mathrm{C}(3)-\mathrm{C}(1)$ & $1.506(2)$ \\
\hline $\mathrm{C}(7)-\mathrm{C}(2)$ & $1.378(2)$ \\
\hline $\mathrm{C}(4)-\mathrm{C}(5)$ & $1.389(2)$ \\
\hline $\mathrm{C}(5)-\mathrm{H}(5 \mathrm{~A})$ & 0.9500 \\
\hline $\mathrm{C}(2)-\mathrm{H}(2)$ & 0.9500 \\
\hline $\mathrm{C}(15)-\mathrm{H}(15)$ & 0.9500 \\
\hline $\mathrm{C}(15)-\mathrm{C}(14)$ & $1.378(2)$ \\
\hline$C(15)-C(16)$ & $1.400(2)$ \\
\hline $\mathrm{C}(14)-\mathrm{C}(13)$ & $1.388(2)$ \\
\hline $\mathrm{C}(16)-\mathrm{C}(17)$ & $1.390(2)$ \\
\hline$C(16)-C(19)$ & $1.507(2)$ \\
\hline $\mathrm{C}(13)-\mathrm{C}(11)$ & $1.511(2)$ \\
\hline $\mathrm{C}(13)-\mathrm{C}(18)$ & $1.384(2)$ \\
\hline $\mathrm{C}(9)-\mathrm{C}(10)$ & $1.555(2)$ \\
\hline $\mathrm{C}(9)-\mathrm{C}(8)$ & $1.520(2)$ \\
\hline $\mathrm{C}(17)-\mathrm{C}(18)$ & $1.392(2)$ \\
\hline $\mathrm{C}(10)-\mathrm{H}(10)$ & 1.0000 \\
\hline $\mathrm{C}(10)-\mathrm{C}(11)$ & $1.525(2)$ \\
\hline
\end{tabular}




\begin{tabular}{|c|c|}
\hline $\mathrm{C}(11)-\mathrm{H}(11)$ & 1.0000 \\
\hline$C(11)-C(12)$ & $1.523(2)$ \\
\hline $\mathrm{C}(18)-\mathrm{H}(18)$ & 0.9500 \\
\hline $\mathrm{C}(1)-\mathrm{H}(1 \mathrm{~A})$ & 0.9800 \\
\hline $\mathrm{C}(1)-\mathrm{H}(1 \mathrm{~B})$ & 0.9800 \\
\hline $\mathrm{C}(1)-\mathrm{H}(1 \mathrm{C})$ & 0.9800 \\
\hline $\mathrm{C}(12)-\mathrm{H}(12 \mathrm{~A})$ & 0.9800 \\
\hline $\mathrm{C}(12)-\mathrm{H}(12 \mathrm{~B})$ & 0.9800 \\
\hline $\mathrm{C}(12)-\mathrm{H}(12 \mathrm{C})$ & 0.9800 \\
\hline $\mathrm{C}(8)-\mathrm{H}(8 \mathrm{~A})$ & 0.9900 \\
\hline $\mathrm{C}(8)-\mathrm{H}(8 \mathrm{~B})$ & 0.9900 \\
\hline $\mathrm{C}(19)-\mathrm{H}(19 \mathrm{~A})$ & 0.9800 \\
\hline $\mathrm{C}(19)-\mathrm{H}(19 \mathrm{~B})$ & 0.9800 \\
\hline C(19)-H(19C) & 0.9800 \\
\hline $\mathrm{C}(7)-\mathrm{O}(2)-\mathrm{C}(10)$ & $107.73(11)$ \\
\hline $\mathrm{C}(14)-\mathrm{O}(1)-\mathrm{C}(9)$ & 114.93(11) \\
\hline $\mathrm{C}(4)-\mathrm{O}(4)-\mathrm{H}(4)$ & 109.5 \\
\hline $\mathrm{C}(17)-\mathrm{O}(5)-\mathrm{H}(5)$ & 109.5 \\
\hline $\mathrm{C}(8)-\mathrm{O}(3)-\mathrm{H}(3)$ & 109.5 \\
\hline$C(7)-C(6)-C(5)$ & $120.13(14)$ \\
\hline$C(7)-C(6)-C(9)$ & $108.90(13)$ \\
\hline$C(5)-C(6)-C(9)$ & $130.96(14)$ \\
\hline$C(4)-C(3)-C(1)$ & $120.77(14)$ \\
\hline$C(2)-C(3)-C(4)$ & $118.87(14)$ \\
\hline$C(2)-C(3)-C(1)$ & $120.36(14)$ \\
\hline $\mathrm{O}(2)-\mathrm{C}(7)-\mathrm{C}(6)$ & $113.57(13)$ \\
\hline $\mathrm{O}(2)-\mathrm{C}(7)-\mathrm{C}(2)$ & $124.30(13)$ \\
\hline$C(2)-C(7)-C(6)$ & $122.13(14)$ \\
\hline $\mathrm{O}(4)-\mathrm{C}(4)-\mathrm{C}(3)$ & $120.80(14)$ \\
\hline $\mathrm{O}(4)-\mathrm{C}(4)-\mathrm{C}(5)$ & $117.42(13)$ \\
\hline$C(5)-C(4)-C(3)$ & $121.73(14)$ \\
\hline$C(6)-C(5)-C(4)$ & $118.30(14)$ \\
\hline$C(6)-C(5)-H(5 A)$ & 120.9 \\
\hline $\mathrm{C}(4)-\mathrm{C}(5)-\mathrm{H}(5 \mathrm{~A})$ & 120.9 \\
\hline
\end{tabular}




\begin{tabular}{|c|c|}
\hline $\mathrm{C}(3)-\mathrm{C}(2)-\mathrm{H}(2)$ & 120.6 \\
\hline$C(7)-C(2)-C(3)$ & $118.84(14)$ \\
\hline $\mathrm{C}(7)-\mathrm{C}(2)-\mathrm{H}(2)$ & 120.6 \\
\hline $\mathrm{C}(14)-\mathrm{C}(15)-\mathrm{H}(15)$ & 119.9 \\
\hline$C(14)-C(15)-C(16)$ & $120.23(14)$ \\
\hline $\mathrm{C}(16)-\mathrm{C}(15)-\mathrm{H}(15)$ & 119.9 \\
\hline $\mathrm{C}(15)-\mathrm{C}(14)-\mathrm{O}(1)$ & $119.76(13)$ \\
\hline$C(15)-C(14)-C(13)$ & $122.06(14)$ \\
\hline $\mathrm{C}(13)-\mathrm{C}(14)-\mathrm{O}(1)$ & $118.11(13)$ \\
\hline$C(15)-C(16)-C(19)$ & $120.63(14)$ \\
\hline$C(17)-C(16)-C(15)$ & $117.72(14)$ \\
\hline $\mathrm{C}(17)-\mathrm{C}(16)-\mathrm{C}(19)$ & $121.65(15)$ \\
\hline $\mathrm{C}(14)-\mathrm{C}(13)-\mathrm{C}(11)$ & $116.86(14)$ \\
\hline $\mathrm{C}(18)-\mathrm{C}(13)-\mathrm{C}(14)$ & $117.94(14)$ \\
\hline $\mathrm{C}(18)-\mathrm{C}(13)-\mathrm{C}(11)$ & $125.19(14)$ \\
\hline $\mathrm{O}(1)-\mathrm{C}(9)-\mathrm{C}(6)$ & $111.40(12)$ \\
\hline $\mathrm{O}(1)-\mathrm{C}(9)-\mathrm{C}(10)$ & $112.63(12)$ \\
\hline $\mathrm{O}(1)-\mathrm{C}(9)-\mathrm{C}(8)$ & $104.45(12)$ \\
\hline$C(6)-C(9)-C(10)$ & $102.07(12)$ \\
\hline $\mathrm{C}(6)-\mathrm{C}(9)-\mathrm{C}(8)$ & $113.02(12)$ \\
\hline $\mathrm{C}(8)-\mathrm{C}(9)-\mathrm{C}(10)$ & $113.55(13)$ \\
\hline $\mathrm{O}(5)-\mathrm{C}(17)-\mathrm{C}(16)$ & $122.67(15)$ \\
\hline $\mathrm{O}(5)-\mathrm{C}(17)-\mathrm{C}(18)$ & $115.82(14)$ \\
\hline $\mathrm{C}(16)-\mathrm{C}(17)-\mathrm{C}(18)$ & $121.50(15)$ \\
\hline $\mathrm{O}(2)-\mathrm{C}(10)-\mathrm{C}(9)$ & $107.05(11)$ \\
\hline $\mathrm{O}(2)-\mathrm{C}(10)-\mathrm{H}(10)$ & 109.1 \\
\hline $\mathrm{O}(2)-\mathrm{C}(10)-\mathrm{C}(11)$ & $108.90(12)$ \\
\hline $\mathrm{C}(9)-\mathrm{C}(10)-\mathrm{H}(10)$ & 109.1 \\
\hline $\mathrm{C}(11)-\mathrm{C}(10)-\mathrm{C}(9)$ & $113.39(13)$ \\
\hline $\mathrm{C}(11)-\mathrm{C}(10)-\mathrm{H}(10)$ & 109.1 \\
\hline $\mathrm{C}(13)-\mathrm{C}(11)-\mathrm{C}(10)$ & $107.45(12)$ \\
\hline $\mathrm{C}(13)-\mathrm{C}(11)-\mathrm{H}(11)$ & 107.2 \\
\hline $\mathrm{C}(13)-\mathrm{C}(11)-\mathrm{C}(12)$ & $114.87(13)$ \\
\hline $\mathrm{C}(10)-\mathrm{C}(11)-\mathrm{H}(11)$ & 107.2 \\
\hline$C(12)-C(11)-C(10)$ & $112.50(13)$ \\
\hline
\end{tabular}




\begin{tabular}{|c|c|}
\hline $\mathrm{C}(12)-\mathrm{C}(11)-\mathrm{H}(11)$ & 107.2 \\
\hline $\mathrm{C}(13)-\mathrm{C}(18)-\mathrm{C}(17)$ & $120.51(15)$ \\
\hline $\mathrm{C}(13)-\mathrm{C}(18)-\mathrm{H}(18)$ & 119.7 \\
\hline $\mathrm{C}(17)-\mathrm{C}(18)-\mathrm{H}(18)$ & 119.7 \\
\hline $\mathrm{C}(3)-\mathrm{C}(1)-\mathrm{H}(1 \mathrm{~A})$ & 109.5 \\
\hline $\mathrm{C}(3)-\mathrm{C}(1)-\mathrm{H}(1 \mathrm{~B})$ & 109.5 \\
\hline $\mathrm{C}(3)-\mathrm{C}(1)-\mathrm{H}(1 \mathrm{C})$ & 109.5 \\
\hline $\mathrm{H}(1 \mathrm{~A})-\mathrm{C}(1)-\mathrm{H}(1 \mathrm{~B})$ & 109.5 \\
\hline $\mathrm{H}(1 \mathrm{~A})-\mathrm{C}(1)-\mathrm{H}(1 \mathrm{C})$ & 109.5 \\
\hline $\mathrm{H}(1 \mathrm{~B})-\mathrm{C}(1)-\mathrm{H}(1 \mathrm{C})$ & 109.5 \\
\hline $\mathrm{C}(11)-\mathrm{C}(12)-\mathrm{H}(12 \mathrm{~A})$ & 109.5 \\
\hline $\mathrm{C}(11)-\mathrm{C}(12)-\mathrm{H}(12 \mathrm{~B})$ & 109.5 \\
\hline $\mathrm{C}(11)-\mathrm{C}(12)-\mathrm{H}(12 \mathrm{C})$ & 109.5 \\
\hline $\mathrm{H}(12 \mathrm{~A})-\mathrm{C}(12)-\mathrm{H}(12 \mathrm{~B})$ & 109.5 \\
\hline $\mathrm{H}(12 \mathrm{~A})-\mathrm{C}(12)-\mathrm{H}(12 \mathrm{C})$ & 109.5 \\
\hline $\mathrm{H}(12 \mathrm{~B})-\mathrm{C}(12)-\mathrm{H}(12 \mathrm{C})$ & 109.5 \\
\hline $\mathrm{O}(3)-\mathrm{C}(8)-\mathrm{C}(9)$ & $111.97(13)$ \\
\hline $\mathrm{O}(3)-\mathrm{C}(8)-\mathrm{H}(8 \mathrm{~A})$ & 109.2 \\
\hline $\mathrm{O}(3)-\mathrm{C}(8)-\mathrm{H}(8 \mathrm{~B})$ & 109.2 \\
\hline $\mathrm{C}(9)-\mathrm{C}(8)-\mathrm{H}(8 \mathrm{~A})$ & 109.2 \\
\hline $\mathrm{C}(9)-\mathrm{C}(8)-\mathrm{H}(8 \mathrm{~B})$ & 109.2 \\
\hline $\mathrm{H}(8 \mathrm{~A})-\mathrm{C}(8)-\mathrm{H}(8 \mathrm{~B})$ & 107.9 \\
\hline $\mathrm{C}(16)-\mathrm{C}(19)-\mathrm{H}(19 \mathrm{~A})$ & 109.5 \\
\hline $\mathrm{C}(16)-\mathrm{C}(19)-\mathrm{H}(19 \mathrm{~B})$ & 109.5 \\
\hline $\mathrm{C}(16)-\mathrm{C}(19)-\mathrm{H}(19 \mathrm{C})$ & 109.5 \\
\hline $\mathrm{H}(19 \mathrm{~A})-\mathrm{C}(19)-\mathrm{H}(19 \mathrm{~B})$ & 109.5 \\
\hline $\mathrm{H}(19 \mathrm{~A})-\mathrm{C}(19)-\mathrm{H}(19 \mathrm{C})$ & 109.5 \\
\hline $\mathrm{H}(19 \mathrm{~B})-\mathrm{C}(19)-\mathrm{H}(19 \mathrm{C})$ & 109.5 \\
\hline
\end{tabular}


SI Table 9. Anisotropic displacement parameters $\left(\AA^{2} \times 10^{3}\right)$ for $x 1-276-1$. The anisotropic displacement factor exponent takes the form: $-2 \pi^{2}\left[h^{2} a^{* 2} U^{11}+\ldots+2 h k a^{*} b^{*} U^{12}\right]$

\begin{tabular}{lllllll}
\hline & $\mathrm{U}^{11}$ & $\mathrm{U}^{22}$ & $\mathrm{U}^{33}$ & $\mathrm{U}^{23}$ & $\mathrm{U}^{13}$ & $\mathrm{U}^{12}$ \\
\hline $\mathrm{O}(2)$ & $23(1)$ & $22(1)$ & $22(1)$ & $-4(1)$ & $3(1)$ & $-6(1)$ \\
$\mathrm{O}(1)$ & $18(1)$ & $17(1)$ & $31(1)$ & $1(1)$ & $5(1)$ & $0(1)$ \\
$\mathrm{O}(4)$ & $31(1)$ & $25(1)$ & $23(1)$ & $-5(1)$ & $-1(1)$ & $-3(1)$ \\
$\mathrm{O}(5)$ & $30(1)$ & $19(1)$ & $50(1)$ & $5(1)$ & $-5(1)$ & $1(1)$ \\
$\mathrm{O}(3)$ & $33(1)$ & $40(1)$ & $26(1)$ & $4(1)$ & $4(1)$ & $-9(1)$ \\
$\mathrm{C}(6)$ & $18(1)$ & $17(1)$ & $21(1)$ & $1(1)$ & $3(1)$ & $2(1)$ \\
$\mathrm{C}(3)$ & $18(1)$ & $21(1)$ & $22(1)$ & $2(1)$ & $2(1)$ & $3(1)$ \\
$\mathrm{C}(7)$ & $17(1)$ & $16(1)$ & $23(1)$ & $-2(1)$ & $-1(1)$ & $2(1)$ \\
$\mathrm{C}(4)$ & $20(1)$ & $18(1)$ & $21(1)$ & $-2(1)$ & $-1(1)$ & $2(1)$ \\
$\mathrm{C}(5)$ & $19(1)$ & $16(1)$ & $25(1)$ & $1(1)$ & $1(1)$ & $-2(1)$ \\
$\mathrm{C}(2)$ & $17(1)$ & $19(1)$ & $26(1)$ & $1(1)$ & $2(1)$ & $-2(1)$ \\
$\mathrm{C}(15)$ & $18(1)$ & $22(1)$ & $26(1)$ & $-3(1)$ & $4(1)$ & $-3(1)$ \\
$\mathrm{C}(14)$ & $18(1)$ & $16(1)$ & $24(1)$ & $0(1)$ & $6(1)$ & $1(1)$ \\
$\mathrm{C}(16)$ & $17(1)$ & $25(1)$ & $25(1)$ & $0(1)$ & $3(1)$ & $0(1)$ \\
$\mathrm{C}(13)$ & $20(1)$ & $20(1)$ & $22(1)$ & $-2(1)$ & $3(1)$ & $1(1)$ \\
$\mathrm{C}(9)$ & $20(1)$ & $17(1)$ & $22(1)$ & $-1(1)$ & $2(1)$ & $0(1)$ \\
$\mathrm{C}(17)$ & $22(1)$ & $17(1)$ & $30(1)$ & $1(1)$ & $5(1)$ & $2(1)$ \\
$\mathrm{C}(10)$ & $21(1)$ & $20(1)$ & $20(1)$ & $0(1)$ & $1(1)$ & $-1(1)$ \\
$\mathrm{C}(11)$ & $25(1)$ & $22(1)$ & $21(1)$ & $-2(1)$ & $2(1)$ & $2(1)$ \\
$\mathrm{C}(18)$ & $23(1)$ & $18(1)$ & $29(1)$ & $-4(1)$ & $1(1)$ & $-1(1)$ \\
$\mathrm{C}(1)$ & $32(1)$ & $33(1)$ & $25(1)$ & $-1(1)$ & $8(1)$ & $-6(1)$ \\
$\mathrm{C}(12)$ & $28(1)$ & $30(1)$ & $26(1)$ & $-7(1)$ & $-2(1)$ & $2(1)$ \\
$\mathrm{C}(8)$ & $29(1)$ & $21(1)$ & $25(1)$ & $3(1)$ & $3(1)$ & $0(1)$ \\
$\mathrm{C}(19)$ & $24(1)$ & $30(1)$ & $37(1)$ & $3(1)$ & $-5(1)$ & $1(1)$ \\
\hline & & & & & & \\
\hline
\end{tabular}


SI Table 10. Hydrogen coordinates (x $\left.10^{4}\right)$ and isotropic displacement parameters $\left(\AA^{2} \times 10^{3}\right)$ for xl-276-1.

\begin{tabular}{|c|c|c|c|c|}
\hline & $\mathrm{x}$ & $\mathrm{y}$ & $\mathrm{z}$ & $\mathrm{U}(\mathrm{eq})$ \\
\hline $\mathrm{H}(4)$ & 6220 & -472 & 1327 & 40 \\
\hline $\mathrm{H}(5)$ & 4515 & 7618 & 3320 & 50 \\
\hline $\mathrm{H}(3)$ & 5495 & -606 & 5467 & 49 \\
\hline $\mathrm{H}(5 \mathrm{~A})$ & 5775 & -789 & 2967 & 24 \\
\hline $\mathrm{H}(2)$ & 7525 & 2816 & 2791 & 25 \\
\hline $\mathrm{H}(15)$ & 4424 & 2621 & 3225 & 26 \\
\hline $\mathrm{H}(10)$ & 6863 & 1723 & 5043 & 24 \\
\hline $\mathrm{H}(11)$ & 5922 & 3105 & 5381 & 27 \\
\hline $\mathrm{H}(18)$ & 5801 & 6300 & 4438 & 28 \\
\hline $\mathrm{H}(1 \mathrm{~A})$ & 7528 & 305 & 1393 & 44 \\
\hline $\mathrm{H}(1 \mathrm{~B})$ & 7725 & 1996 & 1595 & 44 \\
\hline $\mathrm{H}(1 \mathrm{C})$ & 7006 & 1621 & 1123 & 44 \\
\hline $\mathrm{H}(12 \mathrm{~A})$ & 6921 & 5150 & 4910 & 42 \\
\hline $\mathrm{H}(12 \mathrm{~B})$ & 7012 & 4240 & 5669 & 42 \\
\hline $\mathrm{H}(12 \mathrm{C})$ & 6412 & 5466 & 5540 & 42 \\
\hline $\mathrm{H}(8 \mathrm{~A})$ & 5707 & -1116 & 4424 & 30 \\
\hline $\mathrm{H}(8 \mathrm{~B})$ & 6515 & -907 & 4685 & 30 \\
\hline $\mathrm{H}(19 \mathrm{~A})$ & 3835 & 4439 & 2435 & 46 \\
\hline $\mathrm{H}(19 \mathrm{~B})$ & 4121 & 6111 & 2433 & 46 \\
\hline $\mathrm{H}(19 \mathrm{C})$ & 3572 & 5637 & 2999 & 46 \\
\hline
\end{tabular}


SI Table 11. Torsion angles $\left[{ }^{\circ}\right]$ for $x l-276-1$.

\begin{tabular}{|c|c|}
\hline $\mathrm{O}(2)-\mathrm{C}(7)-\mathrm{C}(2)-\mathrm{C}(3)$ & $-179.93(13)$ \\
\hline $\mathrm{O}(2)-\mathrm{C}(10)-\mathrm{C}(11)-\mathrm{C}(13)$ & $69.57(15)$ \\
\hline $\mathrm{O}(2)-\mathrm{C}(10)-\mathrm{C}(11)-\mathrm{C}(12)$ & $-57.82(16)$ \\
\hline $\mathrm{O}(1)-\mathrm{C}(14)-\mathrm{C}(13)-\mathrm{C}(11)$ & $0.7(2)$ \\
\hline $\mathrm{O}(1)-\mathrm{C}(14)-\mathrm{C}(13)-\mathrm{C}(18)$ & $-178.19(13)$ \\
\hline $\mathrm{O}(1)-\mathrm{C}(9)-\mathrm{C}(10)-\mathrm{O}(2)$ & $-111.50(13)$ \\
\hline $\mathrm{O}(1)-\mathrm{C}(9)-\mathrm{C}(10)-\mathrm{C}(11)$ & $8.62(17)$ \\
\hline $\mathrm{O}(1)-\mathrm{C}(9)-\mathrm{C}(8)-\mathrm{O}(3)$ & $-66.90(16)$ \\
\hline $\mathrm{O}(4)-\mathrm{C}(4)-\mathrm{C}(5)-\mathrm{C}(6)$ & $176.84(13)$ \\
\hline $\mathrm{O}(5)-\mathrm{C}(17)-\mathrm{C}(18)-\mathrm{C}(13)$ & $-179.10(14)$ \\
\hline$C(6)-C(7)-C(2)-C(3)$ & $-0.9(2)$ \\
\hline $\mathrm{C}(6)-\mathrm{C}(9)-\mathrm{C}(10)-\mathrm{O}(2)$ & $8.06(15)$ \\
\hline $\mathrm{C}(6)-\mathrm{C}(9)-\mathrm{C}(10)-\mathrm{C}(11)$ & $128.18(13)$ \\
\hline $\mathrm{C}(6)-\mathrm{C}(9)-\mathrm{C}(8)-\mathrm{O}(3)$ & $171.84(13)$ \\
\hline$C(3)-C(4)-C(5)-C(6)$ & $-0.7(2)$ \\
\hline $\mathrm{C}(7)-\mathrm{O}(2)-\mathrm{C}(10)-\mathrm{C}(9)$ & $-7.86(15)$ \\
\hline $\mathrm{C}(7)-\mathrm{O}(2)-\mathrm{C}(10)-\mathrm{C}(11)$ & $-130.81(12)$ \\
\hline$C(7)-C(6)-C(5)-C(4)$ & $0.0(2)$ \\
\hline $\mathrm{C}(7)-\mathrm{C}(6)-\mathrm{C}(9)-\mathrm{O}(1)$ & $114.82(13)$ \\
\hline$C(7)-C(6)-C(9)-C(10)$ & $-5.60(16)$ \\
\hline$C(7)-C(6)-C(9)-C(8)$ & $-127.93(14)$ \\
\hline $\mathrm{C}(4)-\mathrm{C}(3)-\mathrm{C}(2)-\mathrm{C}(7)$ & $0.2(2)$ \\
\hline $\mathrm{C}(5)-\mathrm{C}(6)-\mathrm{C}(7)-\mathrm{O}(2)$ & $179.93(13)$ \\
\hline$C(5)-C(6)-C(7)-C(2)$ & $0.8(2)$ \\
\hline $\mathrm{C}(5)-\mathrm{C}(6)-\mathrm{C}(9)-\mathrm{O}(1)$ & $-63.9(2)$ \\
\hline$C(5)-C(6)-C(9)-C(10)$ & $175.67(15)$ \\
\hline$C(5)-C(6)-C(9)-C(8)$ & $53.3(2)$ \\
\hline $\mathrm{C}(2)-\mathrm{C}(3)-\mathrm{C}(4)-\mathrm{O}(4)$ & $-176.87(13)$ \\
\hline $\mathrm{C}(2)-\mathrm{C}(3)-\mathrm{C}(4)-\mathrm{C}(5)$ & $0.6(2)$ \\
\hline $\mathrm{C}(15)-\mathrm{C}(14)-\mathrm{C}(13)-\mathrm{C}(11)$ & $177.51(14)$ \\
\hline $\mathrm{C}(15)-\mathrm{C}(14)-\mathrm{C}(13)-\mathrm{C}(18)$ & $-1.4(2)$ \\
\hline $\mathrm{C}(15)-\mathrm{C}(16)-\mathrm{C}(17)-\mathrm{O}(5)$ & $177.17(14)$ \\
\hline$C(15)-C(16)-C(17)-C(18)$ & $-1.5(2)$ \\
\hline
\end{tabular}




\begin{tabular}{|c|c|}
\hline $\mathrm{C}(14)-\mathrm{O}(1)-\mathrm{C}(9)-\mathrm{C}(6)$ & $-73.21(15)$ \\
\hline $\mathrm{C}(14)-\mathrm{O}(1)-\mathrm{C}(9)-\mathrm{C}(10)$ & $40.78(16)$ \\
\hline $\mathrm{C}(14)-\mathrm{O}(1)-\mathrm{C}(9)-\mathrm{C}(8)$ & $164.46(12)$ \\
\hline$C(14)-C(15)-C(16)-C(17)$ & $1.9(2)$ \\
\hline$C(14)-C(15)-C(16)-C(19)$ & $-178.17(15)$ \\
\hline$C(14)-C(13)-C(11)-C(10)$ & $46.90(18)$ \\
\hline $\mathrm{C}(14)-\mathrm{C}(13)-\mathrm{C}(11)-\mathrm{C}(12)$ & $172.90(14)$ \\
\hline $\mathrm{C}(14)-\mathrm{C}(13)-\mathrm{C}(18)-\mathrm{C}(17)$ & $1.8(2)$ \\
\hline $\mathrm{C}(16)-\mathrm{C}(15)-\mathrm{C}(14)-\mathrm{O}(1)$ & $176.28(13)$ \\
\hline$C(16)-C(15)-C(14)-C(13)$ & $-0.5(2)$ \\
\hline$C(16)-C(17)-C(18)-C(13)$ & $-0.4(2)$ \\
\hline $\mathrm{C}(9)-\mathrm{O}(1)-\mathrm{C}(14)-\mathrm{C}(15)$ & $135.42(14)$ \\
\hline $\mathrm{C}(9)-\mathrm{O}(1)-\mathrm{C}(14)-\mathrm{C}(13)$ & $-47.66(18)$ \\
\hline $\mathrm{C}(9)-\mathrm{C}(6)-\mathrm{C}(7)-\mathrm{O}(2)$ & $1.04(18)$ \\
\hline$C(9)-C(6)-C(7)-C(2)$ & $-178.05(13)$ \\
\hline $\mathrm{C}(9)-\mathrm{C}(6)-\mathrm{C}(5)-\mathrm{C}(4)$ & $178.61(15)$ \\
\hline $\mathrm{C}(9)-\mathrm{C}(10)-\mathrm{C}(11)-\mathrm{C}(13)$ & $-49.49(17)$ \\
\hline $\mathrm{C}(9)-\mathrm{C}(10)-\mathrm{C}(11)-\mathrm{C}(12)$ & $-176.88(13)$ \\
\hline $\mathrm{C}(10)-\mathrm{O}(2)-\mathrm{C}(7)-\mathrm{C}(6)$ & $4.47(17)$ \\
\hline $\mathrm{C}(10)-\mathrm{O}(2)-\mathrm{C}(7)-\mathrm{C}(2)$ & $-176.47(14)$ \\
\hline $\mathrm{C}(10)-\mathrm{C}(9)-\mathrm{C}(8)-\mathrm{O}(3)$ & $56.18(18)$ \\
\hline $\mathrm{C}(11)-\mathrm{C}(13)-\mathrm{C}(18)-\mathrm{C}(17)$ & $-176.98(14)$ \\
\hline $\mathrm{C}(18)-\mathrm{C}(13)-\mathrm{C}(11)-\mathrm{C}(10)$ & $-134.33(16)$ \\
\hline $\mathrm{C}(18)-\mathrm{C}(13)-\mathrm{C}(11)-\mathrm{C}(12)$ & $-8.3(2)$ \\
\hline $\mathrm{C}(1)-\mathrm{C}(3)-\mathrm{C}(4)-\mathrm{O}(4)$ & $3.3(2)$ \\
\hline $\mathrm{C}(1)-\mathrm{C}(3)-\mathrm{C}(4)-\mathrm{C}(5)$ & $-179.27(15)$ \\
\hline $\mathrm{C}(1)-\mathrm{C}(3)-\mathrm{C}(2)-\mathrm{C}(7)$ & $-179.90(14)$ \\
\hline $\mathrm{C}(8)-\mathrm{C}(9)-\mathrm{C}(10)-\mathrm{O}(2)$ & $130.03(13)$ \\
\hline $\mathrm{C}(8)-\mathrm{C}(9)-\mathrm{C}(10)-\mathrm{C}(11)$ & $-109.85(15)$ \\
\hline $\mathrm{C}(19)-\mathrm{C}(16)-\mathrm{C}(17)-\mathrm{O}(5)$ & $-2.8(2)$ \\
\hline$C(19)-C(16)-C(17)-C(18)$ & $178.59(15)$ \\
\hline
\end{tabular}




\section{DFT study}

\subsection{Computational Methods}

The geometries of reactants, transition states, intermediates and products of $\mathbf{5}+$ cis/trans-o-QM reaction were optimized with M06-2X functional and polarized triple- $\zeta$ 6$311+G(d, p)$ basis sets. Vibrational analyses were performed at same level to verify the stationary points and to obtain their Gibbs free energies (at $298.15 \mathrm{~K}$ ). For multicomponent reactions, the free energies considering entropy effect is more reasonable than electronic potential energy, therefore in the current study, Gibbs free energy is used to evaluate the reaction energy as well as barrier height. Intrinsic reaction coordinate (IRC) calculations were done to ensure the connectivity between TS and minimum structures. During geometry optimization, the polarizable continuum model (PCM) was employed in order to consider the solvent (dichloromethane) contribution to both energy and geometry.

\subsection{Computed free energy surfaces}

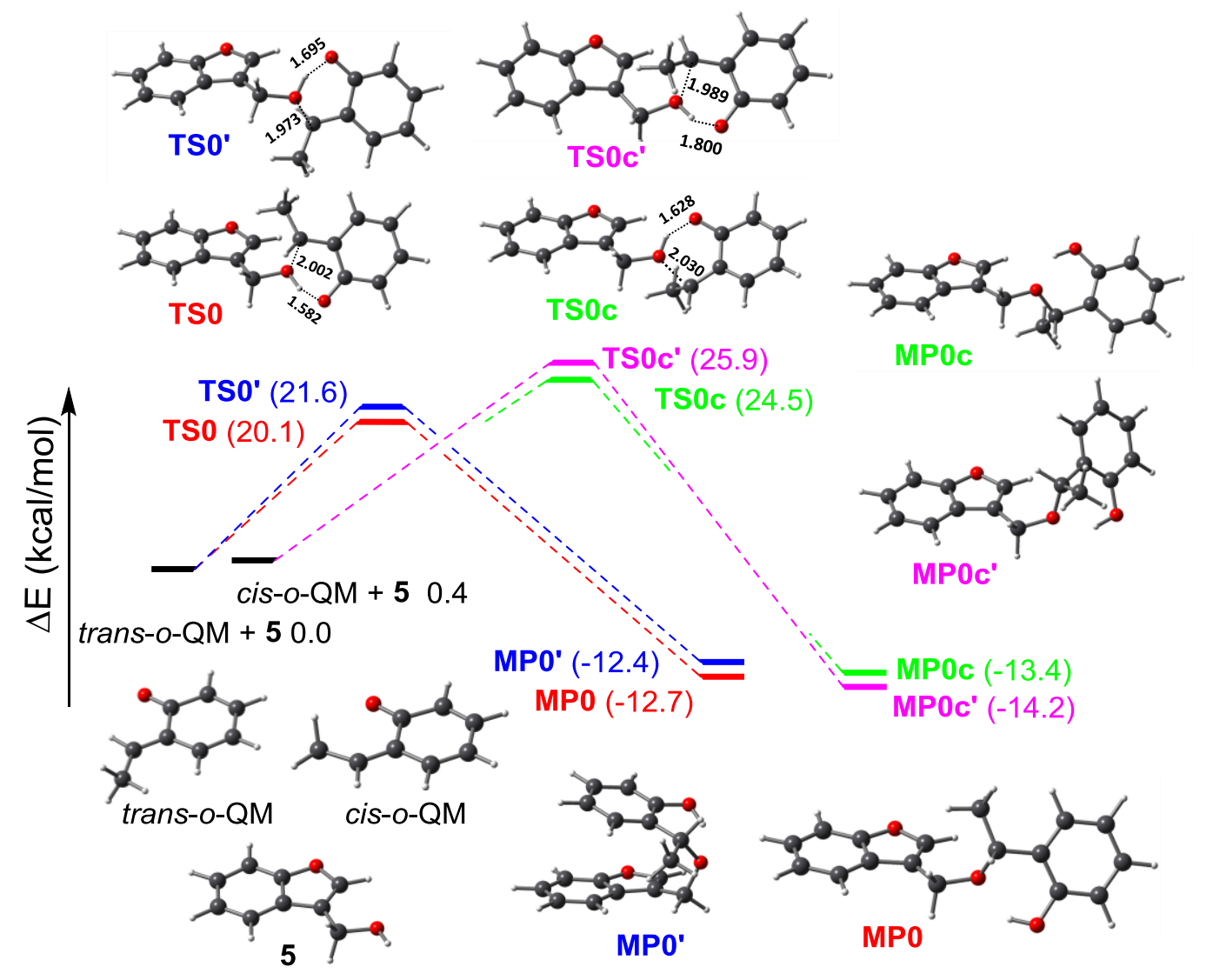

SI Figure 1. Free energy surfaces for oxa-Michael Addition. 


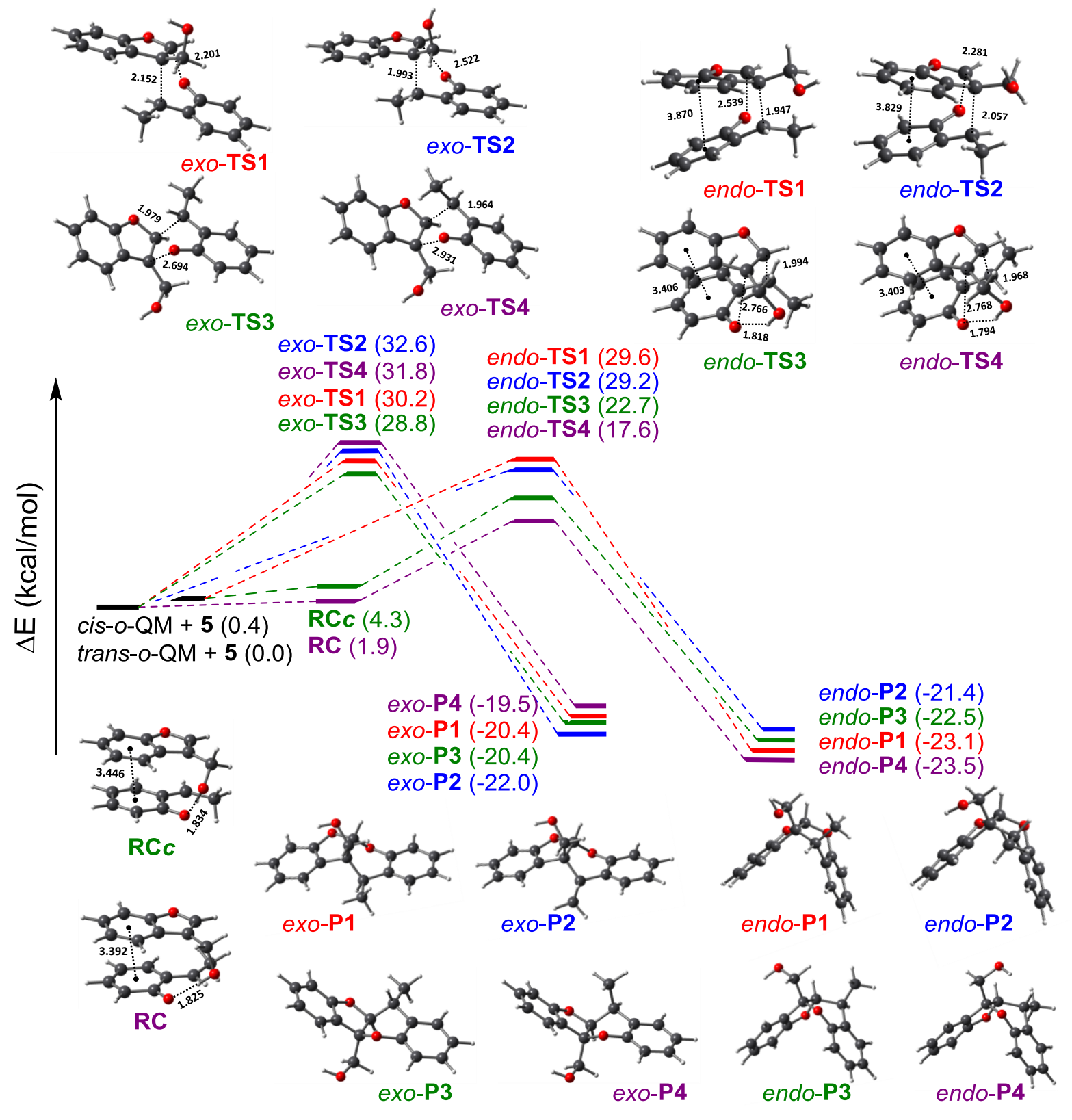

SI Figure 2. Free energy surfaces for [4+2]-cycloaddition. 


\subsection{Cartesian coordinates and Gibbs free energies of all specie}

SI Table 12. Cartesian Coordinates (Angstrom) and Gibbs Free Energies (Hartree) of All Involved Minima and Transition States.

5

$\begin{array}{rrrr} & -498.026382 & & \\ 1 & -0.483939 & -2.506682 & 0.003408 \\ 1 & -2.954128 & -2.332488 & -0.004552 \\ 1 & -4.070534 & -0.137350 & -0.008530 \\ 1 & -2.738334 & 1.972979 & -0.005226 \\ 1 & 2.098978 & 1.991841 & 0.006718 \\ 1 & 2.288530 & -1.617530 & 0.910010 \\ 1 & 2.261729 & -1.655365 & -0.859439 \\ 1 & 4.282825 & -0.861097 & 0.009922 \\ 6 & -0.967182 & -1.536513 & 0.001578 \\ 6 & -2.350564 & -1.432817 & -0.002822 \\ 6 & -2.988246 & -0.181297 & -0.005099 \\ 6 & -2.260323 & 1.001699 & -0.003216 \\ 6 & -0.214974 & -0.355901 & 0.003561 \\ 6 & -0.878883 & 0.875914 & 0.001044 \\ 8 & 0.019921 & 1.895680 & 0.002566 \\ 6 & 1.261259 & 1.315039 & 0.006019 \\ 6 & 1.195945 & -0.032795 & 0.007131 \\ 6 & 2.337264 & -0.992001 & 0.010625 \\ 8 & 3.543716 & -0.248465 & -0.023221\end{array}$

cis-o-QM

$-384.688089$

$-2.900702$

$-3.744502$

$-2.900715$

$-1.729855$

0.514766

1.621620

3.395941

2.858230

$-2.870302$

$-1.615589$

$-0.909264$

0.762559

$-0.340392$

$-0.030638$

1.388675
0.461211

$-0.850502$

0.461203

$-2.085371$

$-2.549051$

2.334287

0.631524

$-1.799648$

$-0.202408$

$-1.002921$

1.774691

$-1.492418$

$-0.542334$

0.916880

1.275958
$-0.868600$

$-0.000012$

0.868597

0.000013

0.000005

0.000003

0.000004

0.000002

$-0.000004$

0.000002

0.000022

0.000000

$-0.000003$

$-0.000019$

$-0.000004$ 
6

6

trs-o-QM

$-384.688693$

$-2.799475$

$-3.624679$

$-3.624750$

$-1.979298$

$-0.816833$

2.687148

3.292924

1.554694

$-3.012419$

$-1.801435$

0.240015

$-0.068819$

$-0.504950$

0.533604

1.930794

2.249627

1.238622

RC

$-882.712047$

$-1.450389$

$-3.027790$

$-2.501023$

0.387489

2.838165

3.809507

2.346073

$-2.473681$

$-2.177649$

$-3.405946$

$-2.263492$

$-2.215644$

0.549361

1.812729

3.520603

2.897507

$-2.149250$

0.331474

$-1.084428$

$-0.000001$

$-0.000002$

$-1.551105$

$-0.245924$

$-0.245793$

1.449724

$-2.176130$

1.412812

$-0.971200$

$-2.749883$

$-0.484938$

0.376685

2.267952

$-1.393013$

$-0.004463$

1.078423

0.636937

$-0.672996$

$-1.714322$

2.773179

2.091828

3.605466

$-2.805313$

$-2.737423$

$-1.522630$

$-0.330811$

$-0.364187$

$-2.828465$

$-2.247408$

1.429988

$-0.509980$

2.943385

0.282510

1.331355

2.667068

2.623828
$-0.000093$

0.874828

$-0.874755$

0.000040

$-0.000040$

0.000002

$-0.000055$

$-0.000068$

$-0.000007$

0.000014

0.000012

$-0.000020$

0.000015

0.000063

0.000006

$-0.000028$

$-0.000036$
$-1.985858$

$-1.534123$

$-0.827040$

1.479445

1.123213

$-0.787596$

$-2.419937$

$-2.092820$

1.000342

$-0.126899$

0.675884

1.752482

$-1.376495$

2.675449

1.251281

$-0.751649$

$-1.164765$ 


\section{RCc}

$-882.708274$

$-0.850493$

4.171089

1.817720

0.715620

3.432844

0.233037

0.446258

$-1.072546$

$-2.428914$

$-1.847004$

$-3.424975$

$-1.658807$

$-1.814177$

$-4.316536$

$-0.382110$

0.095598

$-2.862810$

0.178936

2.045805

1.759264

$-1.198522$

2.323171

1.248085

$-3.061389$

$-1.202564$

2.214529

$-3.371758$

0.238947

2.095434

2.298861

1.737087

2.423234

$-0.957961$

$-1.197010$

$-0.087535$

2.884465

1.840152

$-0.924734$

0.972599

$-2.704489$

$-2.680231$

2.213813

$-1.511425$

$-2.284005$

3.154890

0.760708

1.304811

0.233258

$-1.457178$

$-1.867660$

$-1.003141$

$-2.772758$

$-1.434844$

$-0.978389$

$-3.282410$

$-0.705546$

0.110628 


$\begin{array}{rrrr}6 & -2.488114 & -0.389271 & 1.203833 \\ 6 & -0.638214 & -1.561119 & 0.092071 \\ 6 & -1.172851 & -0.827867 & 1.156256 \\ 8 & -0.226492 & -0.638825 & 2.116912 \\ 6 & 0.905381 & -1.256157 & 1.665908 \\ 6 & 0.735864 & -1.836346 & 0.455234 \\ 6 & 1.756386 & -2.581674 & -0.358437 \\ 8 & 2.817779 & -1.758882 & -0.813689 \\ 6 & 2.047300 & 1.814534 & 0.762576 \\ 8 & 1.408988 & 0.476016 & -1.723538 \\ 6 & -0.278970 & 2.476949 & 0.852771 \\ 6 & 0.818807 & 1.792396 & 0.184846 \\ 6 & 0.566506 & 1.167522 & -1.140340 \\ 6 & -0.745496 & 1.403696 & -1.726735 \\ 6 & -1.703653 & 2.081762 & -1.056980 \\ 6 & -1.478908 & 2.630864 & 0.261031\end{array}$

\section{TS0}

$-882.682985$

$-0.940084$

$-1.984778$

0.990549

$-0.778967$

1.764288

$-1.311287$

$-1.362954$

2.612800

3.375022

2.277431

0.923450

5.787976

1.827059

1.242938

6.803442

$-0.272293$

0.451202

5.421166

$-2.006343$

$-0.695673$

0.677873

$-1.460873$

$-1.461808$

0.552612

1.560544

0.861127

0.687756

2.133795

$-0.803607$

$-1.550369$

0.993912

1.488615

$-1.423425$

1.338968

$-0.614529$

$-3.265346$

$-2.265365$

1.005284

$-5.397850$

1.751329

$-1.042626$

$-6.642259$

$-0.361635$

$-0.814382$

$-5.583363$

$-2.384301$

0.159426

$-0.913544$

$-1.100224$

1.625816

3.812433

1.349782

0.572826

5.164084

1.092313

0.748747

5.744367

$-0.104814$

0.297231

4.988816

$-1.078910$

$-0.342081$

3.032946

0.380227

$-0.068805$

3.639615

$-0.802448$

$-0.506640$ 


$$
\begin{array}{r}
2.720574 \\
1.525733 \\
1.638224 \\
0.545479 \\
-0.699231 \\
-1.741528 \\
-2.845005 \\
-3.728138 \\
-3.014173 \\
-3.575443 \\
-4.938513 \\
-5.616685 \\
-5.017231
\end{array}
$$

$$
\begin{array}{r}
-1.613501 \\
-0.949948 \\
0.252486 \\
1.238224 \\
0.633222 \\
0.004641 \\
1.998791 \\
-1.397754 \\
-0.173265 \\
0.983325 \\
0.881667 \\
-0.302871 \\
-1.464703
\end{array}
$$

$-1.096070$

$-1.036299$

$-0.433425$

$-0.190131$

$-0.510957$

1.078444

$-0.400815$

0.547443

0.498081

$-0.162588$

$-0.587637$

$-0.464577$

0.089540

\section{TS0'}

$-882.680596$

$$
-1.802725
$$

$-0.195845$

$-1.579014$

3.549510

5.875355

6.555789

4.913528

0.287708

0.825521

0.835624

$-0.858526$

$-1.027485$

2.969815

$-0.365742$

2.453641

$-0.950290$

2.411356

$-2.034741$

2.079038

1.184118

1.815155

0.378781

$-0.171848$

$-0.906717$

$-1.975422$

$-1.435769$

$-1.778789$

$-0.027664$

0.777795

2.451525

1.930827

1.121825

0.108703

$-0.974321$

$-0.271460$

1.199139

$-3.946191$

2.167201

$-0.916187$

$-4.360596$

$-2.561804$

0.602729

$-6.362050$

$-1.222829$

0.085953

$-6.177124$

1.147583

$-0.630505$

$-1.267195$

2.260200

$-0.995511$

3.838728

1.201962

0.616684

5.140895

1.048235

0.164573

5.530263

$-0.085258$

$-0.568975$

4.628641

$-1.096960$

$-0.870874$

2.914177

0.193412

0.321496

3.332349

$-0.923649$

$-0.408711$

2.293021

$-1.780904$

$-0.591525$

1.209842

$-1.217352$

0.023115

1.507398

$-0.025073$

0.589333 


$\begin{array}{rrrr}6 & 0.625579 & 0.890531 & 1.380719 \\ 8 & -0.770320 & 0.693896 & 1.170149 \\ 6 & -1.568832 & 0.852456 & -0.627295 \\ 8 & -1.910037 & -1.573667 & 0.567537 \\ 6 & -4.040022 & 1.142013 & -0.574024 \\ 6 & -2.871093 & 0.371740 & -0.356589 \\ 6 & -2.958029 & -0.973768 & 0.163312 \\ 6 & -4.270604 & -1.537113 & 0.261450 \\ 6 & -5.377566 & -0.777974 & -0.018476 \\ 6 & -5.280330 & 0.577670 & -0.425466\end{array}$

TS0c

$-882.676029$

1

1

1

1

1

1

1

1

1

1

1

1

1

1

1

1

6

6

6

6

6

6

6

8

6

6

6

8

6
1.379512

1.231694

$-0.131883$

$-3.331880$

$-5.696354$

$-6.667836$

$-5.290670$

$-0.643067$

$-0.600777$

$-0.704536$

1.013122

1.442776

3.038083

5.440885

6.660780

5.475483

0.958164

$-3.750902$

$-5.074856$

$-5.629778$

$-4.876351$

$-2.974757$

$-3.554826$

$-2.640912$

$-1.477467$

$-1.603361$

$-0.552640$

0.723574

1.519829

$\begin{array}{rr}-1.675611 & 1.637290 \\ -0.308610 & 2.761753 \\ -0.703680 & 1.730496 \\ -2.429869 & 0.003349 \\ -2.091818 & 0.657811 \\ 0.168012 & 0.778912 \\ 2.181146 & 0.253547 \\ 1.881329 & -1.050360 \\ -1.880562 & -0.093309 \\ -1.608392 & -1.834874 \\ 1.273807 & 0.814728 \\ -1.188770 & -0.841262 \\ 2.478474 & 0.604294 \\ -1.737834 & -0.515001 \\ 0.392879 & -0.343973 \\ 2.521755 & 0.145892 \\ -0.681471 & 1.767269 \\ -1.431883 & 0.058498 \\ -1.236435 & 0.422207 \\ 0.052366 & 0.492049 \\ 1.182379 & 0.202504 \\ -0.305617 & -0.238153 \\ 0.964883 & -0.158771 \\ 1.920613 & -0.478898 \\ 1.261345 & -0.765179 \\ -0.077654 & -0.640146 \\ -1.109948 & -0.871172 \\ -0.482691 & -0.882599 \\ 0.309762 & 0.808485\end{array}$

$-1.675611$

1.637290

1.730496

0.003349

0.657811

0.778912

0.253547

$-0.093309$

$-1.834874$

0.814728

0.841262

0.604294

$-0.343973$

0.145892

1.767269

0.058498

0.422207

0.492049

0.202504

0.238153

$-0.158771$

$-0.765179$

$-0.640146$

$-0.871172$

0.808485 
$-1.911473$

$-0.335738$

TSO $c^{\prime}$

0.125153

5.087742

2.246477

0.572457

0.316936

1.543970

0.182379

0.911168

$-1.785864$

$-1.605793$

0.707607

$-2.185840$

0.098429

$-2.060337$

$-2.268032$

0.843554

$-0.892356$

$-0.032960$

$-0.768023$

$-4.375638$

$-2.263966$

0.339214

$-4.150513$

2.598548

$-0.709654$

$-6.292081$

1.391719

$-0.817834$

$-6.421062$

$-1.049298$

$-0.363279$

$-1.137660$

$-0.604128$

1.911200

3.929029

$-1.356755$

$-0.493067$

5.274402

$-1.048811$

$-0.354537$

5.693497

0.236746

0.026400

4.779457

1.251245

0.278915

2.992181

$-0.347542$

$-0.242323$

3.439882

0.922474

0.133727

2.382636

1.755037

0.326681

1.256301

1.020104

0.078776

1.546599

$-0.254027$

$-0.269496$

0.626759

$-1.374191$

$-0.632710$

$-0.750740$

$-1.008968$

$-0.698042$

$-2.000349$

$-1.180764$

0.840376

$-1.842564$

1.405434

$-0.250321$

$-4.340712$

$-1.203044$

0.110499

$-3.116464$

$-0.509567$

0.289672 


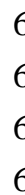

6

6

6

endo-TS1
0.872841

1.545078

0.858566

$-0.533370$

$-5.391289$

$-5.474535$

$-882.667854$

0.478677

2.130720

1.009099

2.227994

0.883964

$-0.999217$

$-1.588328$

0.897734

3.118513

3.601823

1.085466

4.325859

$-0.770735$

$-3.544865$

$-4.532326$

$-3.151810$

1.100616

1.417299

0.652272

$-0.418401$

$-0.757445$

1.101817

0.035940

$-0.109678$

0.808341

1.549703

2.995853

3.378543

0.594096

$-1.027930$

$-1.389671$

$-0.810041$

$-1.578330$

$-2.950774$

$-3.489682$
$-3.330874$

$-2.777016$

$-3.130523$

1.899345

3.996232

4.291729

2.497811

$-1.579893$

$-1.788154$

$-0.120510$

$-0.731654$

$-0.679606$

0.468977

$-1.623870$

0.031254

1.098324

$-2.716609$

2.042526

3.207840

3.378228

2.394174

1.059114

1.266382

0.168317

$-0.716157$

$-0.328927$

$-0.741766$

$-0.533947$

$-1.291394$

$-2.381514$

0.001311

$-0.985434$

$-1.590956$

$-1.177363$

$-0.251003$
$-0.120051$

$-0.460686$

$-0.530984$

$-0.269984$
$-1.473199$

$-1.165602$

0.184773

$-1.381562$

$-1.384133$

0.170076

1.816676

2.269250

0.634357

1.004251

$-1.704224$

$-1.086065$

$-2.391172$

1.158481

$-0.381276$

$-2.158114$

$-0.815834$

$-0.678137$

$-0.678434$

0.205817

1.131461

0.252495

1.115464

1.954265

1.631080

0.488476

0.337580

$-1.010352$

$-0.909305$

1.080036

$-1.630246$

$-0.803767$

0.256897

0.368338

$-0.488893$ 
endo-TS2

$-882.668542$

$-2.203053$

$-0.726165$

$-1.213306$

$-2.434952$

$-1.372857$

0.509084

1.361633

$-0.648700$

$-2.662377$

$-3.191664$

$-0.477130$

$-3.963169$

0.934861

3.565251

4.481921

3.168964

$-1.182799$

$-1.622115$

$-1.014516$

0.051325

0.535525

$-1.158605$

$-0.104253$

0.195593

$-0.584929$

$-1.405593$

$-2.766813$

$-3.661492$

$-0.345241$

1.173861

1.510384

0.976320

1.717642

3.005468

3.501505

2.754687
$-1.537388$

$-1.663165$

$-0.100742$

1.484899

3.717452

4.231768

2.500658

$-1.815716$

$-2.355161$

$-1.543039$

$-2.488959$

0.130030

0.409976

$-1.234538$

0.504374

1.331341

$-1.170425$

1.701629

2.956016

3.250743

2.301296

0.739506

1.079265

0.021142

$-0.998955$

$-0.697427$

$-1.377857$

$-0.630890$

$-1.493530$

$-2.305555$

0.040132

$-0.989089$

$-1.470724$

$-0.886597$

0.081941

0.559182
2.115914

3.076146

2.419444

1.238138

1.238353

$-0.265015$

$-1.865735$

$-2.377011$

0.048003

$-1.421564$

0.594855

$-0.134796$

2.538095

$-1.359149$

0.139132

2.077605

2.211248

0.557332

0.556249

$-0.298417$

$-1.195561$

$-0.340440$

$-1.184844$

$-2.021084$

$-1.677917$

$-0.564367$

$-0.427682$

0.370994

1.008576

$-1.041933$

1.696272

0.883799

$-0.255648$

$-0.498210$

0.334045

1.441193

endo-TS3 


\begin{tabular}{|c|c|c|c|}
\hline 1 & 2.988418 & 2.715820 & 0.951090 \\
\hline 1 & 3.513170 & 1.033028 & 0.903974 \\
\hline 1 & 2.819138 & 1.783598 & -0.543608 \\
\hline 1 & -0.450903 & -2.462897 & -1.888008 \\
\hline 1 & -2.915468 & -2.467447 & -1.650741 \\
\hline 1 & -3.994137 & -1.555588 & 0.365520 \\
\hline 1 & -2.652400 & -0.603001 & 2.231876 \\
\hline 1 & 2.178462 & -0.556261 & 2.108784 \\
\hline 1 & 2.884647 & -2.608311 & -0.043746 \\
\hline 1 & 1.911251 & -2.311561 & -1.487897 \\
\hline 1 & 1.224262 & 1.641337 & 2.067324 \\
\hline 1 & 2.734741 & -0.129842 & -1.332326 \\
\hline 1 & -0.920245 & 2.393851 & 1.914018 \\
\hline 1 & -1.164024 & 1.045406 & -2.861559 \\
\hline 1 & -3.127703 & 2.025702 & -1.740857 \\
\hline 1 & -3.017772 & 2.719594 & 0.648963 \\
\hline 6 & 2.757348 & 1.728641 & 0.538680 \\
\hline 6 & -0.925291 & -2.079865 & -0.992759 \\
\hline 6 & -2.291861 & -2.077403 & -0.856237 \\
\hline 6 & -2.912301 & -1.547502 & 0.303459 \\
\hline 6 & -2.184451 & -1.022332 & 1.351183 \\
\hline 6 & -0.152670 & -1.543486 & 0.062077 \\
\hline 6 & -0.799268 & -1.027250 & 1.199884 \\
\hline 8 & 0.092830 & -0.568072 & 2.094615 \\
\hline 6 & 1.326483 & -0.562342 & 1.442440 \\
\hline 6 & 1.227329 & -1.314887 & 0.253951 \\
\hline 6 & 2.345706 & -1.836903 & -0.601595 \\
\hline 8 & 3.277912 & -0.845049 & -0.954939 \\
\hline 6 & 1.366214 & 1.385427 & 1.017661 \\
\hline 8 & 1.127279 & 0.629661 & -1.710008 \\
\hline 6 & -0.977591 & 2.121942 & 0.864023 \\
\hline 6 & 0.193339 & 1.609274 & 0.241396 \\
\hline 6 & 0.146304 & 1.192678 & -1.150520 \\
\hline 6 & -1.111158 & 1.368971 & -1.827904 \\
\hline 6 & -2.197745 & 1.905684 & -1.194300 \\
\hline 6 & -2.142990 & 2.300064 & 0.168536 \\
\hline
\end{tabular}

endo-TS4

$-882.686995$

$1-1.181191$

$-2.874329$

2.488525

$-2.170487$

1

$-1.793249$

2.374698

$-1.658069$

3.542614

$-0.890546$ 


\begin{tabular}{|c|c|c|c|}
\hline 1 & 0.414455 & -2.995185 & 1.146623 \\
\hline 1 & 2.843859 & -2.997424 & 0.664014 \\
\hline 1 & 3.786324 & -1.546659 & -1.088781 \\
\hline 1 & 2.337989 & -0.040599 & -2.439101 \\
\hline 1 & -2.451439 & 0.075507 & -1.822696 \\
\hline 1 & -2.005104 & -2.571798 & 1.131700 \\
\hline 1 & -3.125727 & -2.412764 & -0.227304 \\
\hline 1 & -2.285182 & 1.274051 & 0.431539 \\
\hline 1 & -2.612178 & -0.403470 & 1.656803 \\
\hline 1 & 0.729574 & 2.907324 & -1.078967 \\
\hline 1 & 1.540010 & 0.006244 & 2.880279 \\
\hline 1 & 3.381859 & 1.298921 & 1.872652 \\
\hline 1 & 2.977192 & 2.778237 & -0.088085 \\
\hline 6 & -1.853162 & 2.535571 & -1.312586 \\
\hline 6 & 0.828238 & -2.375358 & 0.360657 \\
\hline 6 & 2.174317 & -2.370046 & 0.089035 \\
\hline 6 & 2.716378 & -1.531142 & -0.917829 \\
\hline 6 & 1.927923 & -0.694544 & -1.681003 \\
\hline 6 & -0.006796 & -1.529808 & -0.405642 \\
\hline 6 & 0.563260 & -0.712471 & -1.401457 \\
\hline 8 & -0.378715 & 0.013199 & -2.030120 \\
\hline 6 & -1.541459 & -0.138097 & -1.277969 \\
\hline 6 & -1.379262 & -1.209315 & -0.373563 \\
\hline 6 & -2.462915 & -1.856055 & 0.441218 \\
\hline 8 & -3.254542 & -0.913649 & 1.128071 \\
\hline 6 & -1.478864 & 1.536016 & -0.244491 \\
\hline 8 & -0.924326 & 0.072239 & 2.036805 \\
\hline 6 & 0.903026 & 2.283257 & -0.209000 \\
\hline 6 & -0.185967 & 1.563488 & 0.349819 \\
\hline 6 & 0.021392 & 0.732492 & 1.523534 \\
\hline 6 & 1.365484 & 0.655016 & 2.029295 \\
\hline 6 & 2.380789 & 1.372439 & 1.459856 \\
\hline 6 & 2.157251 & 2.213136 & 0.337190 \\
\hline
\end{tabular}

exo-TS1

$-882.666910$

0.525280

$-2.703872$

$-0.980270$

$-0.907199$

$-1.958036$

$-1.726133$

$-2.032531$

$-2.606984$

$-2.493571$

$-2.326299$

$-0.007384$

$-4.718332$

$-1.729043$

$-0.912432$

$-5.361820$

0.624847

$-1.245487$ 


\begin{tabular}{|c|c|c|c|}
\hline 1 & -3.796953 & 2.472315 & -0.614831 \\
\hline 1 & 0.368420 & 1.660988 & 1.613261 \\
\hline 1 & 1.174626 & -1.006019 & 1.731209 \\
\hline 1 & 0.042026 & -2.223877 & 1.129693 \\
\hline 1 & 0.056734 & 0.304825 & -1.555150 \\
\hline 1 & -1.399681 & -1.459658 & 2.799516 \\
\hline 1 & 2.732709 & -2.269617 & -0.921323 \\
\hline 1 & 3.751817 & 2.458931 & 0.285743 \\
\hline 1 & 5.511546 & 0.726957 & 0.364106 \\
\hline 1 & 5.003239 & -1.631928 & -0.222551 \\
\hline 6 & 0.172875 & -1.888992 & -1.613213 \\
\hline 6 & -2.765600 & -1.286286 & -0.148778 \\
\hline 6 & -4.016097 & -0.945110 & -0.656846 \\
\hline 6 & -4.384036 & 0.391826 & -0.842264 \\
\hline 6 & -3.522965 & 1.431773 & -0.499308 \\
\hline 6 & -1.883029 & -0.259110 & 0.184479 \\
\hline 6 & -2.299108 & 1.060845 & 0.021624 \\
\hline 8 & -1.339849 & 1.924333 & 0.504791 \\
\hline 6 & -0.310061 & 1.181196 & 0.928538 \\
\hline 6 & -0.505415 & -0.195914 & 0.692002 \\
\hline 6 & 0.116758 & -1.233321 & 1.589232 \\
\hline 8 & -0.464313 & -1.249698 & 2.886552 \\
\hline 6 & 0.593748 & -0.535807 & -1.127369 \\
\hline 8 & 1.259675 & 1.957142 & -0.404529 \\
\hline 6 & 2.951834 & -1.239994 & -0.660178 \\
\hline 6 & 1.929336 & -0.257986 & -0.766634 \\
\hline 6 & 2.204879 & 1.111067 & -0.408182 \\
\hline 6 & 3.538322 & 1.430926 & 0.015712 \\
\hline 6 & 4.503938 & 0.463447 & 0.060434 \\
\hline 6 & 4.217181 & -0.888927 & -0.273497 \\
\hline
\end{tabular}

exo-TS2

$-882.663106$

$-0.241178$

$-2.112107$

0.790648

0.380868

$-1.769476$

2.396785

$-1.207280$

$-1.140506$

1.943170

$-2.297943$

2.145262

1.254458

$-4.571324$

1.306470

1.790151

$-5.391609$

$-0.816505$

0.854965

$-3.961114$

$-2.169191$

$-0.689014$

0.410985

$-0.763203$

$-2.106926$

1.242218

1.670881

$-1.133648$ 


$\begin{array}{lrrr}1 & 0.336670 & 2.436589 & 0.174613 \\ 1 & 0.213714 & 0.771202 & 1.726214 \\ 1 & -0.506481 & 2.301504 & -2.509762 \\ 1 & 2.339259 & 1.698750 & 1.876825 \\ 1 & 4.136789 & -1.752675 & -1.213137 \\ 1 & 5.606867 & 0.016878 & -0.329464 \\ 1 & 4.720518 & 1.760118 & 1.211357 \\ 6 & -0.206024 & -1.355682 & 1.570874 \\ 6 & -2.658454 & 1.215884 & 0.829042 \\ 6 & -3.929836 & 0.736768 & 1.129097 \\ 6 & -4.399331 & -0.469861 & 0.594563 \\ 6 & -3.617716 & -1.232844 & -0.269071 \\ 6 & -1.866574 & 0.465390 & -0.034312 \\ 6 & -2.369500 & -0.718631 & -0.557484 \\ 8 & -1.426476 & -1.302774 & -1.390897 \\ 6 & -0.355019 & -0.526765 & -1.387681 \\ 6 & -0.466520 & 0.555047 & -0.486000 \\ 6 & 0.236030 & 1.872516 & -0.754552 \\ 8 & -0.495138 & 2.703397 & -1.635545 \\ 6 & 0.500350 & -0.096260 & 1.129585 \\ 8 & 1.581231 & -1.959894 & -0.640586 \\ 6 & 2.742758 & 0.941312 & 1.210296 \\ 6 & 1.889156 & -0.099647 & 0.774077 \\ 6 & 2.370443 & -1.100829 & -0.146589 \\ 6 & -1.005964 & -0.525994 \\ 6 & -0.014010 & 0.841452 \\ 6 & 4.754160 & 0.981937 & \end{array}$

exo-TS3

$-882.669146$

0.924695

$-0.112467$

3.567828

0.103974

$-1.624173$

3.537874

$-0.810703$

2.292601

$-2.522516$

$-0.089879$

4.471381

$-2.036184$

$-1.168291$

5.273202

0.278816

$-1.410349$

3.921867

2.199584

$-0.574879$

$-0.142941$

1.549012

1.900475

$-1.274072$

$-1.172651$

1.222336

$-0.283995$

$-1.160510$

2.667983

$-0.130042$

1.396293

$-1.088684$

0.825288

$-2.921345$

1.884063 
1.988776

0.591316

$-2.483444$

$-1.467951$

$-1.866561$

$-0.592979$

0.361227

0.439098

3.062482

0.005589

$-1.508634$

$-0.170596$

$-1.227325$

$-0.778937$

0.099094

$-0.922343$

1.177276

$-0.463229$

$-0.432090$

0.320915

0.877910

0.161064

1.785341

0.688474

1.081030

1.087280

$-0.299037$

0.975485

$-1.327081$

1.584398

$-2.654158$

1.286623

1.605035

$-0.329894$

$-0.896694$

$-1.355325$

1.016895

0.143324

0.714924

$-0.378120$

$-0.588420$

$-0.976769$

$-1.507812$

$-1.028650$

$-1.158282$

$-0.537242$

0.114134

0.059659

\section{exo-TS4}

$\begin{array}{rrr}-882.664375 & & \\ -0.672171 & -1.073821 & -1.550988 \\ 0.532876 & -2.187187 & -2.157163 \\ -0.752001 & -2.799351 & -1.098761 \\ -2.161990 & 2.509432 & -0.392045 \\ -4.431698 & 1.987468 & -1.238741 \\ -5.366173 & -0.280163 & -1.036103 \\ -4.062882 & -2.121327 & 0.022891 \\ 0.185909 & -1.321176 & 2.104630 \\ 1.403934 & 1.176266 & 1.036778 \\ 0.412766 & 1.492402 & 2.442406 \\ 1.003154 & -2.629362 & 0.465486 \\ -0.532982 & 3.172663 & 1.378636 \\ 3.130891 & -2.117079 & 1.171741 \\ 3.188106 & 1.977542 & -1.649873 \\ 5.193957 & 1.304321 & -0.383617\end{array}$


$-0.733777$

1.047835

$-1.955319$

$-1.289393$

1.521358

$-0.276559$

1.218884

$-0.754594$

$-0.083808$

$-0.643198$

$-1.117578$

$-0.056671$

0.492088

0.342858

$-0.796747$

0.435769

$-1.656227$

1.042241

$-0.968240$

1.246555

0.393936

0.937731

1.460693

1.345695

2.728736

0.806231

$-1.722557$

$-0.113226$

0.682846

$-1.579751$

$-1.224788$

0.551586

$-0.904147$

$-0.205553$

0.304351

$-1.012712$

1.076658

$-1.046076$

0.698994

$-0.334475$

$-0.460392$

0.486786

\section{MP0}

$-882.735364$

$-0.831280$

$-1.871751$

0.938660

0.126717

$-0.756537$

1.926567

$-1.449115$

$-1.293759$

2.503260

3.402980

2.199946

1.044224

5.846283

1.806393

1.122833

6.836219

$-0.224642$

0.143116

5.399814

$-1.941883$

$-0.958699$

0.595999

$-1.487910$

$-1.289227$

0.577452

1.401780

1.194503

0.646552

2.089126

$-0.426116$

$-1.607219$

1.005124

1.643860

$-2.022756$

1.912679

$-0.649069$

$-3.156431$

$-2.054403$

1.254066

$-5.369778$

1.502254

$-1.362522$

$-6.600675$

$-0.592615$

$-0.818745$

$-5.466004$

$-2.381398$

0.485103

$-0.885342$

$-1.013962$

1.611850

3.829802

1.302623

0.611473 


5.197941
5.763779
4.977591
3.019424
3.612702
2.664700
1.464281
1.600045
0.518451
-0.767644
-1.544029
-2.980783
-3.637001
-2.939856
-3.591332
-4.902402
-5.580274
-4.946935

1.077182

0.652334

$-0.080588$

0.093207

$-1.044964$

$-0.523828$

0.342123

$-0.005104$

$-0.799853$

$-0.554698$

$-1.604992$

$-1.104380$

$-0.978074$

$-0.905650$

0.193049

$-0.248932$

1.153978

0.127758

0.640829

$-0.193319$

0.171201

0.928377

2.068955

$-0.670827$

$-1.271614$

0.680460

$-0.096912$

0.400146

0.894161

$-0.353884$

0.713553

$-0.784956$

$-0.459655$

$-0.479015$

$-1.460707$

0.249862

\section{MPO'}

$-882.734788$

1.513027

$-1.201170$

2.457574

3.218938

$-1.628190$

2.212593

2.781065

$-0.054776$

2.892359

$-0.832741$

$-1.726363$

2.100905

$-3.015893$

$-0.772080$

2.766220

$-4.481052$

0.319409

1.115330

$-3.807422$

0.471618

$-1.284792$

0.371080

$-1.295673$

$-2.931846$

1.229454

$-2.587616$

0.741325

1.687626

$-2.958892$

$-0.919086$

3.476071

0.234762

0.599637

2.461219

0.094173

$-1.573208$

0.675324

0.928550

2.524033

0.281294

2.736493

$-2.057710$

$-1.218637$

3.575210

$-0.252792$

$-1.006492$

2.658855

2.047627

$-0.816189$

2.166424

$-1.471579$

$-1.251495$

1.364717

$-2.695482$

$-0.714790$

1.732760

$-3.532671$

$-0.092329$

0.791551

$-3.171973$

0.000047

$-0.545963$ 
$-1.160170$

0.023860

$-0.549154$

$-0.890592$

$-0.580850$

$-2.140731$

$-1.202800$

$-2.019930$

$-1.562946$

$-0.745505$

$-2.157602$

$-0.254557$

$-1.239077$

$-0.253350$

$-0.220406$

0.768624

0.972777

$-1.794890$

1.331833

1.520935

0.847629

0.522014

1.386406

$-0.769403$

1.315118

2.360972

$-1.044422$

$-0.472135$

2.821154

$-0.031197$

$-0.354331$

2.310322

1.256285

MP0c

$-882.737718$

2.018802

1.886507

1.850873

2.839619

2.939954

0.684967

1.084917

3.035850

0.863702

$-3.502804$

2.355741

0.169929

$-5.953167$

1.986546

0.201525

$-6.896539$

$-0.276709$

$-0.025574$

$-5.408157$

$-2.261181$

$-0.289983$

$-0.584215$

$-1.896535$

$-0.378798$

$-0.619218$

1.744225

$-0.931979$

$-0.746462$

1.705040

0.835816

1.767148

$-1.195768$

$-0.564123$

1.337886

1.438721

$-1.918496$

$-2.174395$

1.393132

$-1.693893$

$-0.257384$

0.119409

$-1.932058$

2.354204

0.866407

1.356784

0.070378

1.144104

0.087289

$-0.146784$

$-0.042080$

$-1.261133$

$-0.189998$

$-5.005811$

0.246748

$-0.077966$

$-1.026821$

$-0.202778$

$-3.638620$

$-1.964838$

$-0.331362$ 
$-1.471562$

$-1.642070$

$-0.583241$

0.674168

1.807767

2.283636

4.037671

3.029727

3.215486

4.393308

5.390624

5.216741
$-1.291376$

0.039384

1.085237

0.437836

1.286305

$-0.946757$

0.645553

0.397015

$-0.636257$

$-1.381543$

$-1.106672$

$-0.091785$
$-0.292354$

$-0.143393$

$-0.053914$

0.003198

$-0.217655$

1.612594

$-1.191672$

$-0.261669$

0.669367

0.663037

$-0.263125$

$-1.200412$

\section{MP0 $c^{\prime}$}

$-882.737802$

$-2.890640$

$-2.557634$

$-1.379308$

3.730051

5.956736

6.263839

4.334231

$-0.149942$

0.839173

0.889162

$-0.658426$

$-2.290034$

$-1.901096$

$-4.778857$

$-5.034807$

$-3.575585$

$-2.106416$

3.856095

5.101026

5.277125

4.213288

2.769861

2.977365

1.808052

0.852001

1.356836

0.604784
2.761416

2.107736

3.183045

2.205986

1.170896

$-1.264899$

$-2.757503$

$-1.074159$

2.379988

2.389236

0.788119

1.521921

$-1.017405$

$-0.881085$

$-2.730529$

$-2.806066$

2.386881

1.132883

0.547581

$-0.841786$

$-1.685296$

0.300048

$-1.079962$

$-1.712455$

$-0.737032$

0.495763

1.790938
0.709115

2.324586

1.545361

0.253151

0.576305

0.381152

$-0.143686$

$-0.812173$

0.505718

$-1.258484$

1.419798

$-1.532163$

2.226021

$-1.786720$

$-0.145550$

1.868498

1.370771

0.167701

0.346424

0.236306

$-0.055611$

$-0.125913$

$-0.229672$

$-0.515752$

$-0.596272$

$-0.373652$

$-0.390320$

S44 
8

endo-P1

$\begin{array}{rr}1.575876 & -0.485504 \\ 1.177101 & 0.741879 \\ 1.070060 & -1.625679 \\ -0.993305 & 1.350195 \\ 0.060186 & 0.448004 \\ 0.081979 & -0.691638 \\ -0.923692 & -0.895775 \\ -1.954661 & 0.024988 \\ -1.998386 & 1.153343\end{array}$

$-882.751895$

1.889081

0.298021

1.395176

$-1.903487$

$-2.918029$

$-2.938879$

$-1.936600$

$-0.455055$

$-1.275195$

$-2.557429$

0.058162

$-2.920723$

1.941416

3.054066

4.281886

3.712531

1.041347

$-1.911644$

$-2.482470$

$-2.494917$

$-1.939948$

$-1.363810$

$-1.380306$

$-0.798009$

$-0.182379$

$-0.699368$

$-1.733377$

$-2.190972$

0.457479

1.212062

\begin{tabular}{rr}
3.013865 & -1.084399 \\
3.611415 & -0.601113 \\
2.911702 & 0.609832 \\
0.051471 & -2.247578 \\
-2.216580 & -2.438436 \\
-3.736738 & -0.497370 \\
-3.032415 & 1.678958 \\
1.385586 & 2.434453 \\
3.034527 & 0.888089 \\
1.811883 & 1.022502 \\
1.437494 & -1.722215 \\
3.131336 & -0.819418 \\
0.136593 & -2.672995 \\
-1.305142 & 1.938351 \\
-2.282965 & -0.006202 \\
-1.564006 & -2.308985 \\
2.833022 & -0.421270 \\
-0.623131 & -1.399352 \\
-1.894569 & -1.500501 \\
-2.752663 & -0.402198 \\
-2.372387 & 0.820855 \\
-0.234979 & -0.187903 \\
-1.108701 & 0.889394 \\
-0.578879 & 2.009053 \\
0.664539 & 1.664796 \\
1.052256 & 0.244718 \\
2.169736 & 0.394008 \\
2.511482 & -0.901084 \\
1.441992 & -0.705003 \\
0.530631 & 1.773337 \\
& \\
\hline &
\end{tabular}


$-0.181002$

$-1.666948$

1.522041

0.380845

$-0.588640$

1.850640

$-0.049113$

0.695325

2.833105

$-1.000878$

0.922399

3.510874

$-1.539191$

$-0.168563$

3.191612

$-1.134760$

$-1.461770$

endo-P2

$-882.749179$

1
0.805432

$-0.890478$

0.215507

1.796318

2.534727

2.403678

1.522690

0.541940

1.555055

2.516266

$-0.732954$

3.236268

$-2.042810$

$-3.152414$

$-4.460345$

$-3.884258$

$-0.041451$

1.723983

2.146130

2.074310

1.584766

1.224752

1.172402

0.691370

0.194965

0.736747

1.931990

2.763832

$-0.415739$

$-1.205488$

$-2.285494$

$-1.566747$

$-1.901443$
$-3.105534$

$-3.029414$

$-1.729361$

$-0.287255$

1.994083

3.828847

3.404103

$-0.928576$

$-2.838757$

$-1.407118$

$-2.502448$

$-1.398182$

$-1.016466$

1.375087

1.823164

0.629395

$-2.438280$

0.511713

1.800771

2.834272

2.611794

0.276957

1.327246

0.948951

$-0.384628$

$-0.976536$

$-1.887603$

$-2.196621$

$-1.732208$

$-0.364936$

$-0.496158$

$-0.764474$

$-0.065689$
$-1.347841$

$-1.849021$

$-2.287303$

$-2.185436$

$-2.787740$

$-1.141597$

1.157317

2.730967

1.251237

1.659844

0.517037

$-0.491385$

$-2.416806$

1.785786

$-0.298842$

$-2.391042$

$-1.498471$

$-1.458382$

$-1.795622$

$-0.865323$

0.422202

$-0.183672$

0.726418

1.948532

1.853164

0.511198

0.865818

$-0.233172$

$-0.200464$

1.974825

$-1.497686$

$-0.338483$

0.821825 
6

6

6

endo-P3
0.861674

1.103884

0.432144
0.858588

$-0.309070$

$-1.483151$

$-882.750876$

1

1

1

1

1

1

1

1

1

1

1

1

1

1

1

1

6

6

6

6

6

6

6

8

6

6

6

8

6

8

6

6

6

6

6

6
$-1.834234$

$-3.087256$

$-1.567599$

2.278157

3.615525

3.541769

2.144635

0.543567

0.555984

1.501937

$-1.637715$

3.043308

$-3.362349$

$-1.493029$

$-3.299122$

$-4.220872$

$-2.009677$

2.262531

3.004590

2.960169

2.185444

1.493275

1.457278

0.643978

0.103403

0.571803

1.251806

2.388250

$-1.415574$

$-0.500335$

$-2.963420$

$-1.972718$

$-1.461012$

$-1.922784$

$-2.928569$

$-3.446186$

$\begin{array}{rr}2.663861 & -0.603759 \\ 2.641883 & 0.640866 \\ 3.502812 & 0.937042 \\ -0.720802 & -2.200446 \\ -2.618183 & -1.289943 \\ -3.140962 & 1.118016 \\ -1.793630 & 2.677870 \\ 2.270341 & 1.227992 \\ 2.977566 & -1.275191 \\ 1.831957 & -2.245522 \\ 1.306052 & 2.143895 \\ 1.879534 & -0.511804 \\ -0.473113 & 1.931377 \\ -1.527683 & -2.538248 \\ -2.957591 & -1.570511 \\ -2.429396 & 0.669146 \\ 2.617877 & 0.473054 \\ -0.951962 & -1.140359 \\ -2.015493 & -0.629666 \\ -2.310263 & 0.735433 \\ -1.564482 & 1.620649 \\ -0.200048 & -0.263511 \\ -0.508835 & 1.088220 \\ 0.306091 & 1.805117 \\ 1.317849 & 0.923543 \\ 0.964283 & -0.519159 \\ 2.137741 & -1.223903 \\ 2.586310 & -0.518367 \\ 1.336444 & 1.074076 \\ 0.595048 & -1.402254 \\ -0.708082 & 0.949804 \\ 0.104114 & 0.408295 \\ -0.218037 & -0.848965 \\ -1.311859 & -1.567315 \\ -2.102303 & 0.240049 \\ -1.805650 & \end{array}$


$-882.752465$

exo-P1
$-1.798644$

$-1.783224$

$-3.209316$

2.682336

4.156631

3.914981

2.205706

0.291271

1.655129

1.943110

$-1.881293$

$-0.496843$

$-3.333493$

$-0.939516$

$-2.624361$

$-3.803463$

$-2.118625$

2.591626

3.411090

3.271445

2.322085

1.651233

1.520810

0.545225

$-0.012209$

0.620492

1.185426

0.178241

$-1.534160$

$-0.340195$

$-2.813497$

$-1.894754$

$-1.232040$

$-1.478125$

$-2.418114$

$-3.080212$
0.509595

2.270815

1.349815

$-0.291975$

$-2.050716$

$-2.704444$

$-1.625929$

2.292291

2.114331

2.769180

2.036163

2.964234

$-0.874513$

$-1.591643$

$-3.259775$

$-2.892183$

1.336561

$-0.576900$

$-1.564965$

$-1.934139$

$-1.337916$

0.028753

$-0.354411$

0.316555

1.316103

1.081773

2.356083

3.335681

1.197827

0.625363

$-1.028064$

$-0.083100$

$-0.319145$

$-1.449802$

$-2.373977$

$-2.167354$
2.921968

2.740034

2.253038

$-2.075459$

$-1.106128$

1.259199

2.714695

1.280487

$-2.076074$

$-0.449122$

0.266855

$-1.848281$

1.553370

$-2.728788$

$-1.938118$

0.210475

2.285209

$-1.032897$

$-0.489328$

0.850480

1.677755

$-0.212733$

1.114623

1.778831

0.893865

$-0.508848$

$-1.115761$

$-1.269167$

0.885011

$-1.486578$

0.614978

0.171252

$-1.035422$

$-1.799545$

$-1.349168$

$-0.143279$

2.936084

$-0.757573$ 


\begin{tabular}{|c|c|c|c|}
\hline 1 & -1.191871 & 2.949163 & -1.444896 \\
\hline 1 & -0.987084 & 2.972476 & 0.307669 \\
\hline 1 & 2.442211 & 2.339921 & -0.410074 \\
\hline 1 & 4.709173 & 1.677057 & -1.125825 \\
\hline 1 & 5.356438 & -0.704231 & -1.197609 \\
\hline 1 & 3.727684 & -2.477664 & -0.533477 \\
\hline 1 & -0.339467 & -1.412645 & 1.510277 \\
\hline 1 & -0.750674 & 1.019593 & 2.115172 \\
\hline 1 & 0.620503 & 2.063504 & 1.690586 \\
\hline 1 & -0.295869 & 0.695182 & -1.622713 \\
\hline 1 & 1.974738 & 0.386772 & 2.485733 \\
\hline 1 & -3.169325 & 2.280654 & -0.391190 \\
\hline 1 & -3.304431 & -2.675735 & -0.217986 \\
\hline 1 & -5.425655 & -1.353717 & -0.116905 \\
\hline 1 & -5.334626 & 1.120522 & -0.183645 \\
\hline 6 & -0.588488 & 2.560913 & -0.622429 \\
\hline 6 & 2.703875 & 1.289716 & -0.456158 \\
\hline 6 & 3.989807 & 0.915376 & -0.852155 \\
\hline 6 & 4.354980 & -0.428734 & -0.889182 \\
\hline 6 & 3.455517 & -1.429855 & -0.524236 \\
\hline 6 & 1.789321 & 0.302545 & -0.112114 \\
\hline 6 & 2.188480 & -1.028089 & -0.139280 \\
\hline 8 & 1.213679 & -1.888479 & 0.281844 \\
\hline 6 & 0.031088 & -1.159212 & 0.513900 \\
\hline 6 & 0.356794 & 0.364257 & 0.376155 \\
\hline 6 & 0.283503 & 1.024664 & 1.759556 \\
\hline 8 & 1.043508 & 0.317232 & 2.723660 \\
\hline 6 & -0.622597 & 1.035321 & -0.632643 \\
\hline 8 & -0.908764 & -1.626238 & -0.443983 \\
\hline 6 & -3.194013 & 1.198112 & -0.352184 \\
\hline 6 & -2.011202 & 0.469120 & -0.431689 \\
\hline 6 & -2.089312 & -0.922816 & -0.371628 \\
\hline 6 & -3.296824 & -1.593321 & -0.258844 \\
\hline 6 & -4.471769 & -0.846972 & -0.201623 \\
\hline 6 & -4.419908 & 0.542797 & -0.238730 \\
\hline
\end{tabular}

exo-P2

$-882.750059$

0.184829

$-0.184812$

$-2.284639$ 


\begin{tabular}{|c|c|c|c|}
\hline 1 & -4.704666 & -1.840171 & -0.812681 \\
\hline 1 & -5.349219 & 0.519360 & -1.135576 \\
\hline 1 & -3.712245 & 2.353647 & -0.709742 \\
\hline 1 & 0.315294 & 1.485354 & 1.470270 \\
\hline 1 & 0.767192 & -0.862958 & 2.276936 \\
\hline 1 & -0.573254 & -1.973021 & 1.930051 \\
\hline 1 & 0.639196 & -2.228951 & -0.046467 \\
\hline 1 & -1.970405 & -0.257864 & 2.589655 \\
\hline 1 & 3.102877 & -2.456108 & -0.058107 \\
\hline 1 & 3.410410 & 2.486500 & -0.344201 \\
\hline 1 & 5.487332 & 1.114184 & -0.141939 \\
\hline 1 & 5.324299 & -1.353016 & 0.019826 \\
\hline 6 & 0.251790 & -1.203360 & -1.895338 \\
\hline 6 & -2.689570 & -1.384612 & -0.213767 \\
\hline 6 & -3.980534 & -1.055957 & -0.630374 \\
\hline 6 & -4.343660 & 0.278400 & -0.811747 \\
\hline 6 & -3.439263 & 1.314370 & -0.579329 \\
\hline 6 & -1.778591 & -0.362484 & 0.011016 \\
\hline 6 & -2.166815 & 0.956938 & -0.166671 \\
\hline 8 & -1.175657 & 1.850769 & 0.129476 \\
\hline 6 & -0.008413 & 1.140260 & 0.484641 \\
\hline 6 & -0.346182 & -0.385113 & 0.486374 \\
\hline 6 & -0.266032 & -0.920939 & 1.924469 \\
\hline 8 & -1.042220 & -0.160718 & 2.829974 \\
\hline 6 & 0.633821 & -1.194894 & -0.406866 \\
\hline 8 & 0.981953 & 1.511395 & -0.461546 \\
\hline 6 & 3.178884 & -1.375041 & -0.118039 \\
\hline 6 & 2.019550 & -0.617875 & -0.250923 \\
\hline 6 & 2.134264 & 0.769767 & -0.323815 \\
\hline 6 & 3.366680 & 1.405682 & -0.284459 \\
\hline 6 & 4.518065 & 0.630944 & -0.170146 \\
\hline 6 & 4.426207 & -0.755500 & -0.079106 \\
\hline
\end{tabular}

exo-P3

$-882.747664$

$-1.782182$

3.035307

1.199972

$-0.340965$

3.612443

0.346292

$-1.885925$

3.507172

$-0.504744$

2.230648

$-2.236708$

$-1.095981$

4.536006

$-1.514404$

$-1.715972$

5.334186

0.738155

$-1.109334$

1

3.852723

2.332260

0.105972 
$-0.352385$

$-0.724164$

0.875457

$-0.394781$

1.753250

$-3.693197$

$-2.483954$

$-4.885416$

$-5.474043$

$-1.253644$

2.598193

3.884947

4.333240

3.517007

1.787763

2.243188

1.316870

0.060810

0.393809

0.323872

0.795207

$-0.908533$

$-0.461479$

$-3.429047$

$-2.089510$

$-1.781887$

$-2.769511$

$-4.104320$

$-4.434717$
1.008132

$-1.520554$

$-0.954497$

1.615207

$-2.701560$

2.070366

$-2.676848$

$-1.996161$

0.378316

3.024681

$-1.255297$

$-0.843395$

0.432470

1.334369

$-0.369333$

0.903964

1.660452

0.951228

$-0.516193$

$-1.428804$

$-2.733435$

1.600434

$-1.039040$

1.033245

0.666276

$-0.680612$

$-1.644535$

$-1.254153$

0.078913
1.830188

1.947332

2.466753

$-1.140667$

1.305279

$-0.029300$

$-0.802037$

$-0.688903$

$-0.288622$

0.242616

$-0.817915$

$-1.169871$

$-0.828235$

$-0.145685$

$-0.124264$

0.189352

0.841624

0.819140

0.418154

1.646452

1.396297

$-0.169100$

$-0.605667$

$-0.200323$

$-0.285646$

$-0.494490$

$-0.639478$

$-0.573101$

$-0.348315$

\section{exo-P4}

$\begin{array}{rrr}-882.746151 & & \\ 0.024406 & -1.449434 & -1.639932 \\ 1.040419 & -2.886933 & -1.453625 \\ -0.576234 & -2.848790 & -0.730993 \\ -2.059959 & 2.015923 & -1.436170 \\ -4.381691 & 1.272977 & -1.965920 \\ -5.285162 & -0.785454 & -0.953458 \\ -3.898308 & -2.161987 & 0.593654 \\ 0.317409 & -0.667310 & 2.137613 \\ 0.788371 & 1.790253 & 1.790556 \\ -0.854408 & 1.402162 & 2.340926\end{array}$




$\begin{array}{rrrr}1 & 1.220483 & -2.519773 & 0.978244 \\ 1 & -1.602506 & 2.922712 & 0.816420 \\ 1 & 3.646799 & -2.222625 & 0.586227 \\ 1 & 2.664733 & 2.301716 & -1.188387 \\ 1 & 5.031341 & 1.542344 & -0.944391 \\ 1 & 5.514988 & -0.716357 & -0.043590 \\ 6 & 0.308791 & -2.250099 & -0.953844 \\ 6 & -2.474717 & 1.123640 & -0.980726 \\ 6 & -3.770305 & 0.698401 & -1.281503 \\ 6 & -4.278556 & -0.467160 & -0.708880 \\ 6 & -3.515510 & -1.245587 & 0.162628 \\ 6 & -1.716920 & 0.362264 & -0.104908 \\ 6 & -2.232660 & -0.804767 & 0.443218 \\ 8 & -1.349485 & -1.453401 & 1.253378 \\ 6 & -0.063682 & -0.813271 & 1.124584 \\ 6 & -0.327120 & 0.558696 & 0.433939 \\ 6 & -0.252472 & 1.686370 & 1.469694 \\ 8 & -0.649858 & 2.936762 & 0.955419 \\ 6 & 0.919664 & -1.686837 & 0.337486 \\ 8 & 0.570812 & 0.859422 & -0.639446 \\ 6 & 3.441806 & -1.230856 & 0.196108 \\ 6 & 2.120083 & -0.823070 & 0.042756 \\ 6 & 1.870142 & 0.457885 & -0.452301 \\ 6 & 2.902967 & 1.315724 & -0.807975 \\ 6 & 4.217908 & 0.882634 & -0.160410 \\ 6 & 4.490176 & -0.385915 & \end{array}$




\section{References}

[1]. Macías, F. A.; Marin, D.; Chinchilla, D.; Molinillo, J. M. G. Tetrahedron Letters 2002, 43, 6417. 
6. Copies of ${ }^{1} \mathrm{H}$ and ${ }^{13} \mathrm{C}$-NMR spectra of new compounds

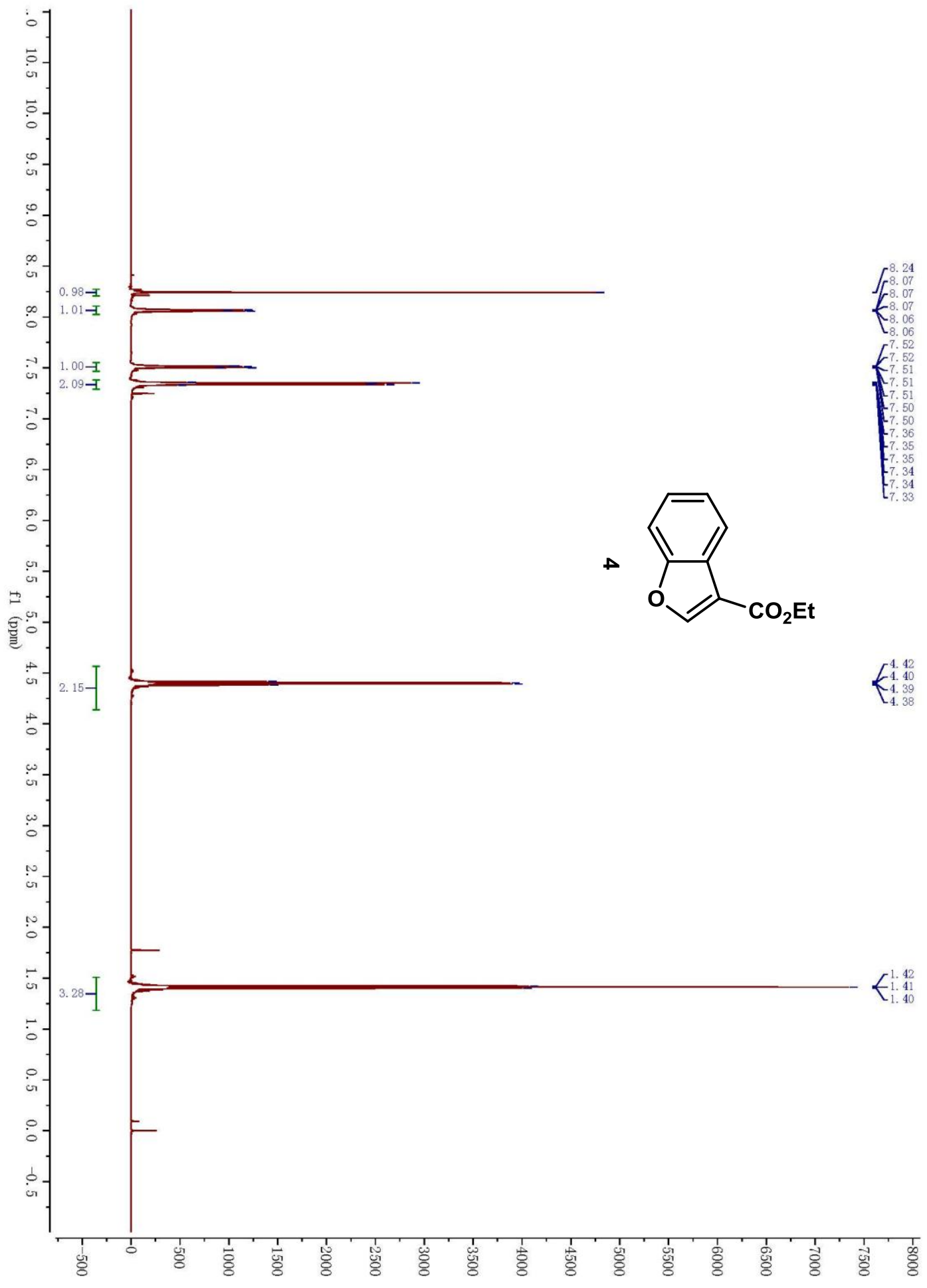




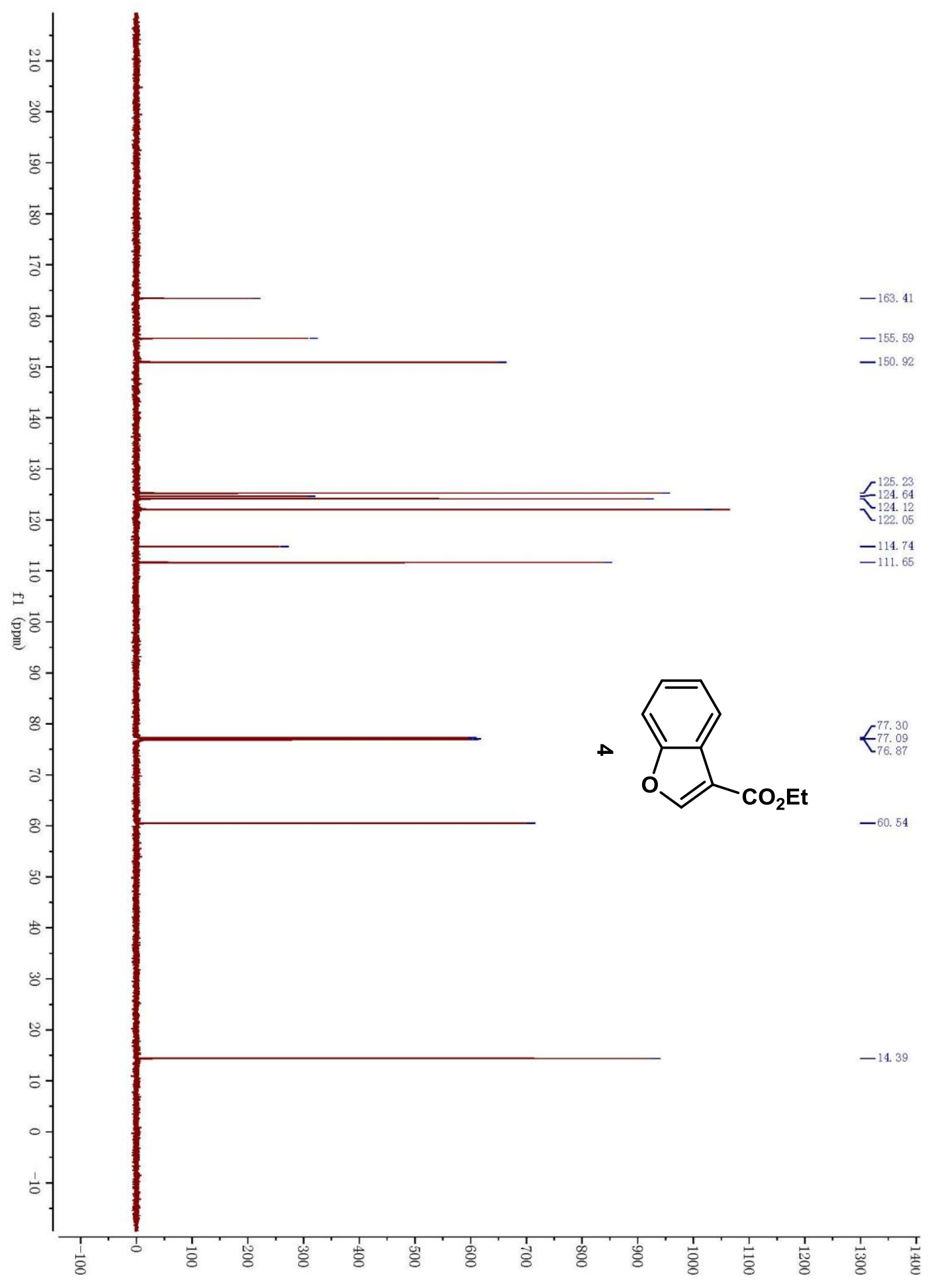




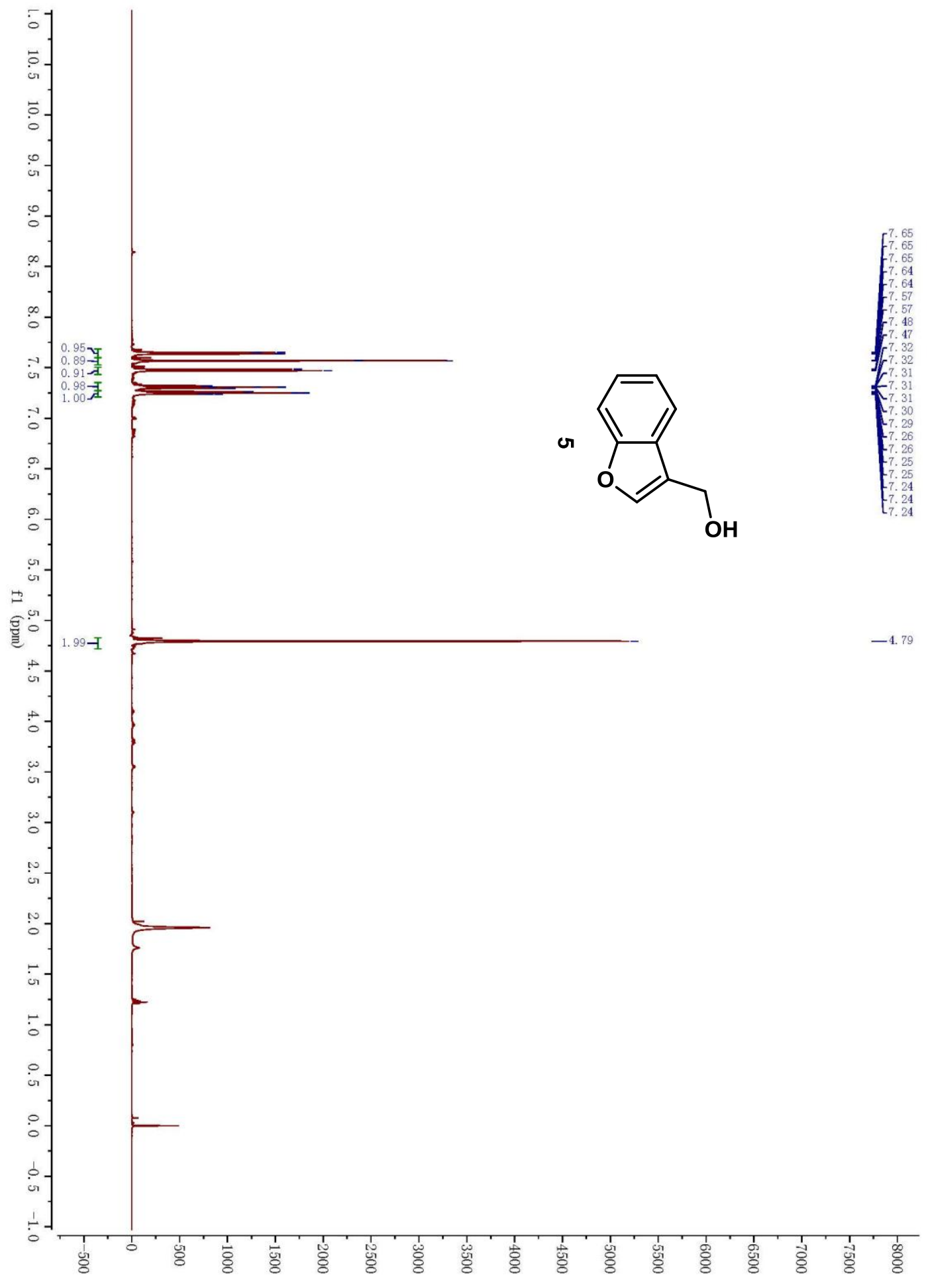




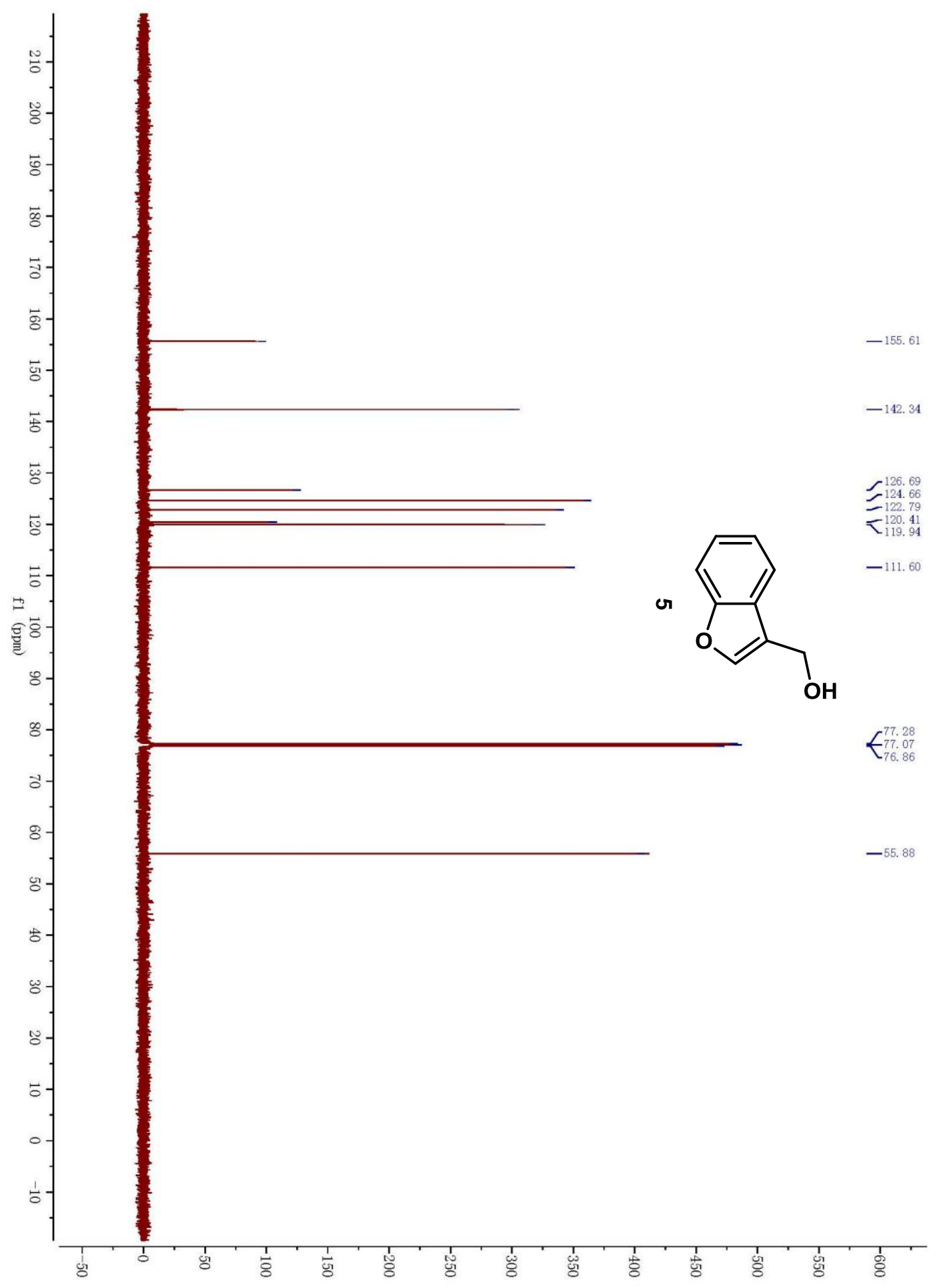




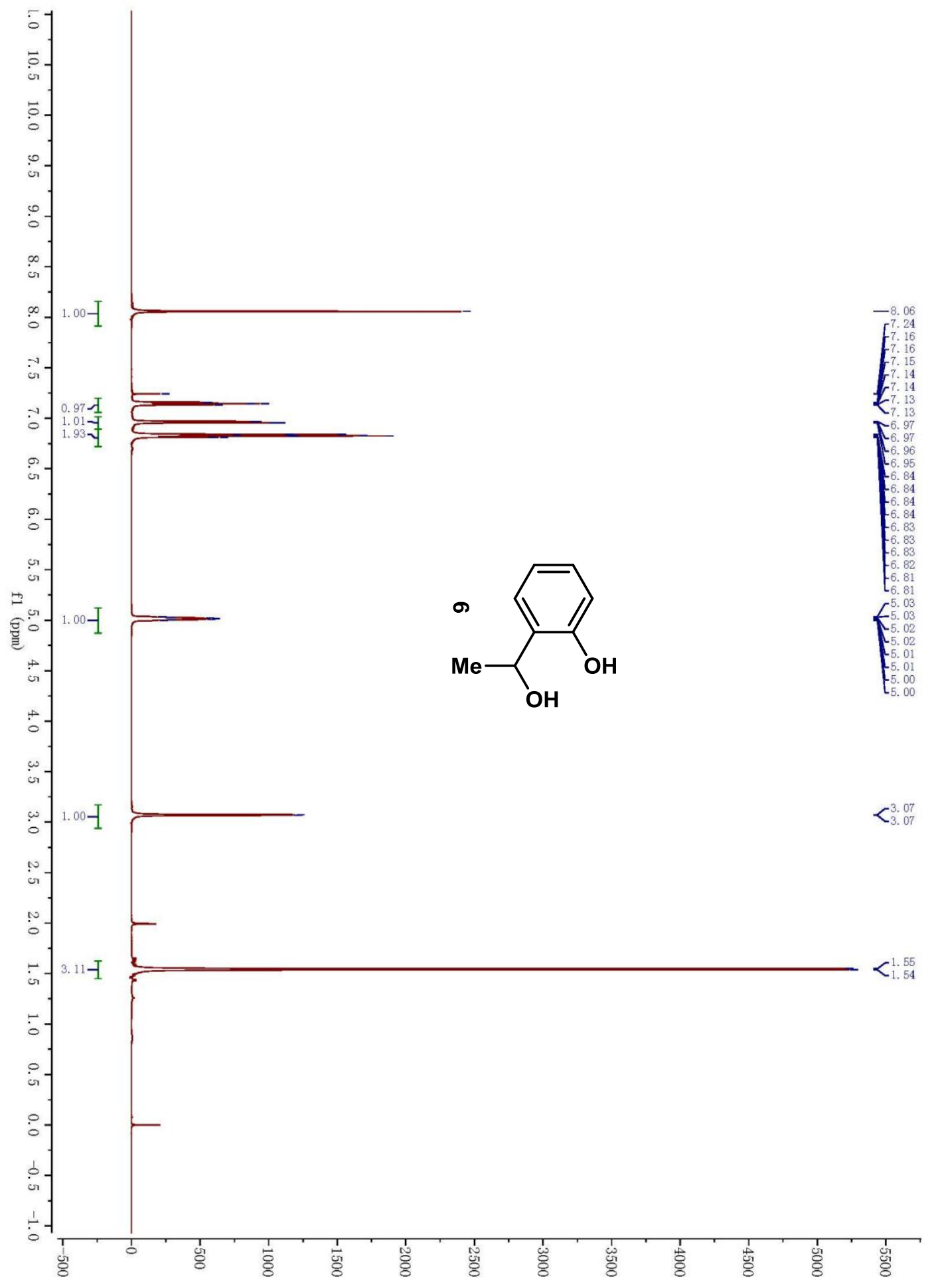




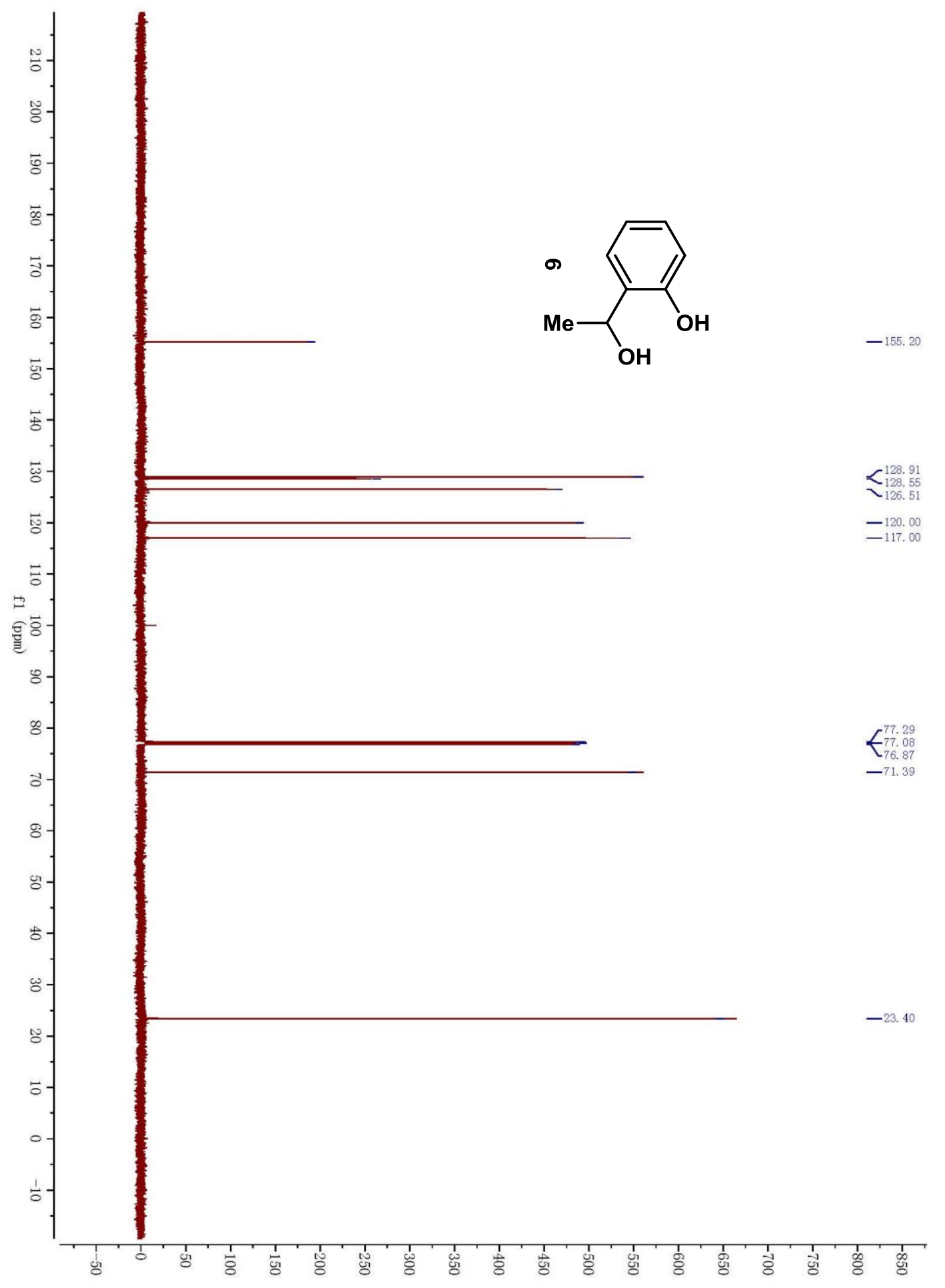




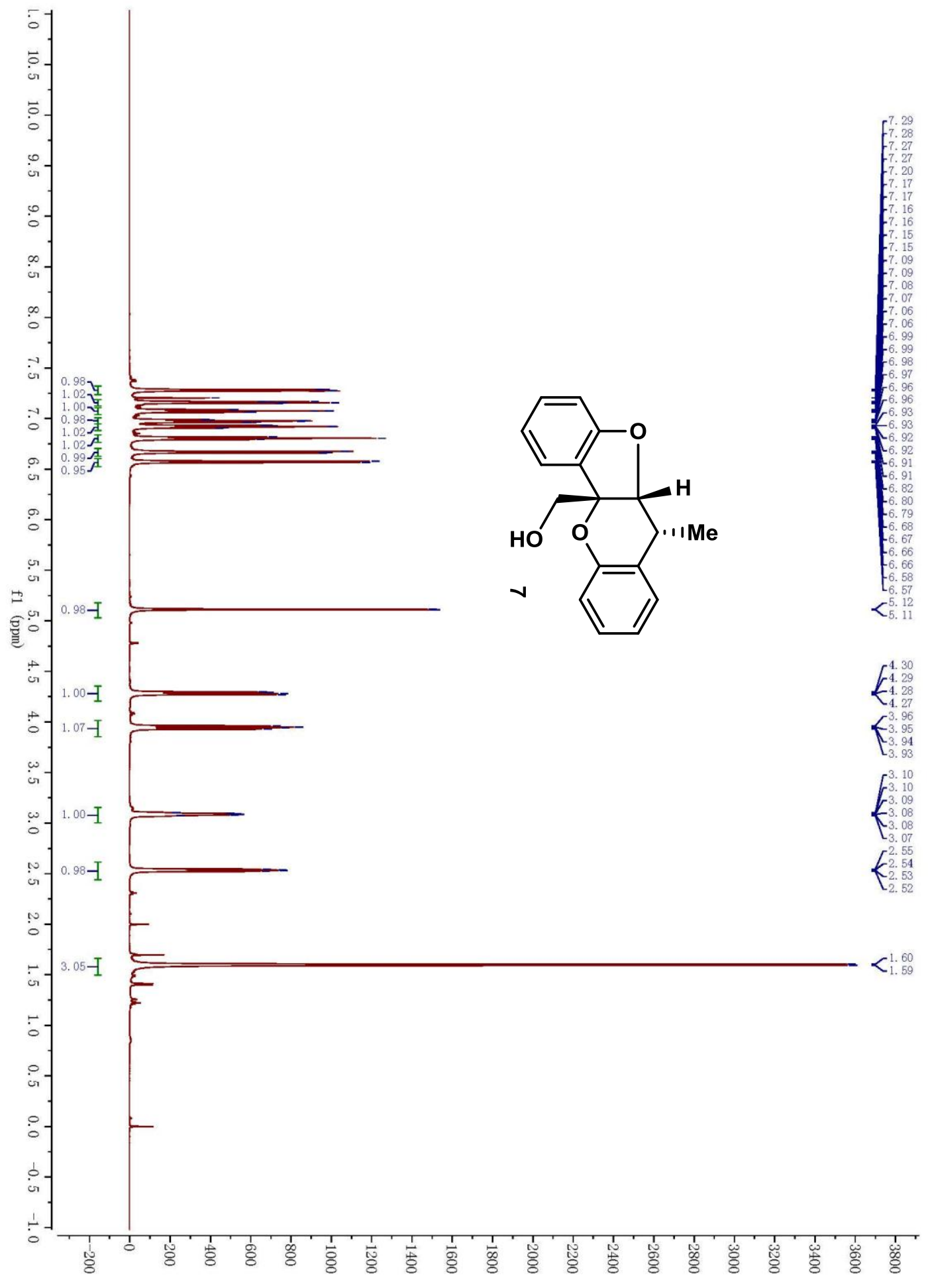




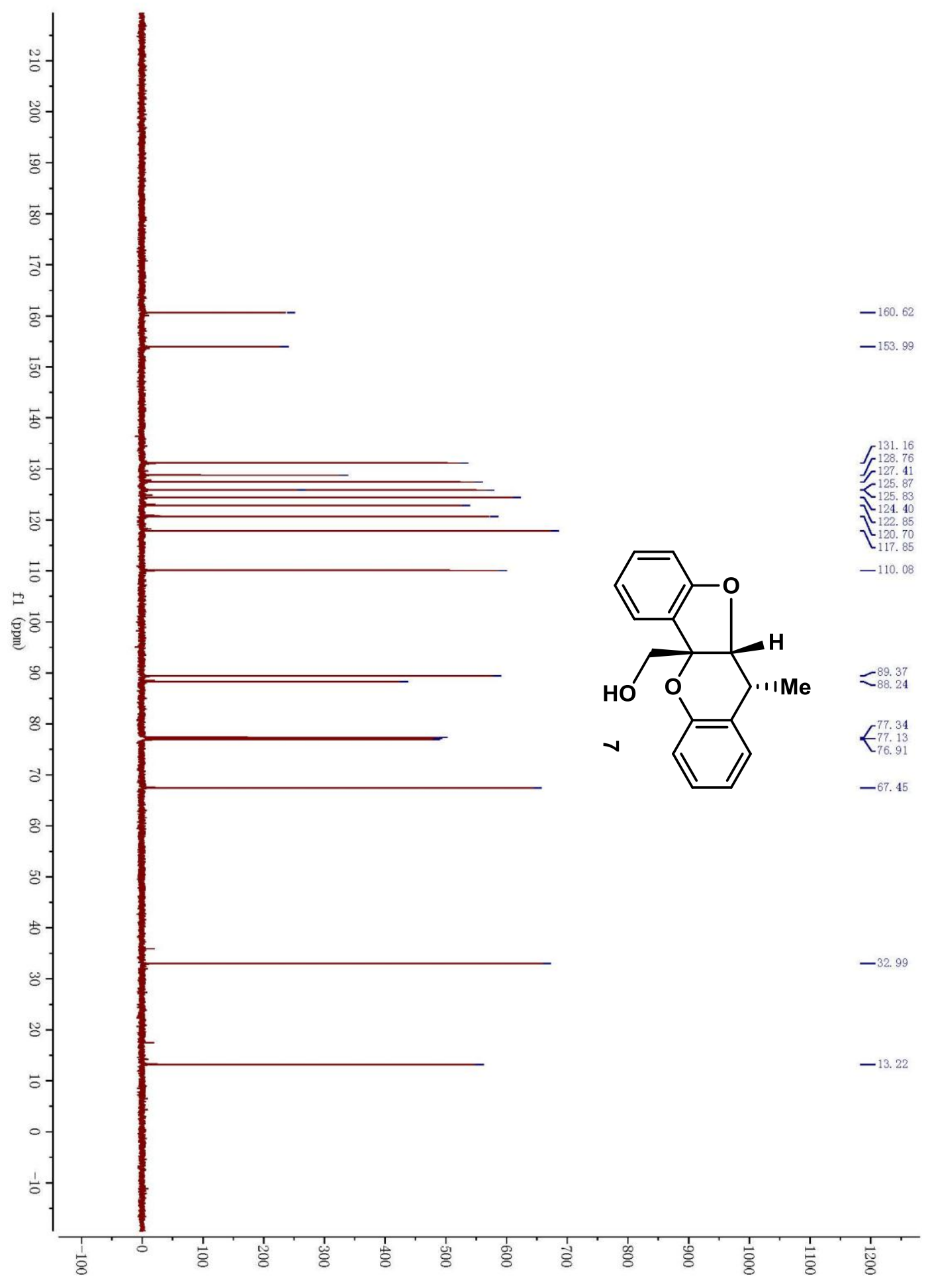




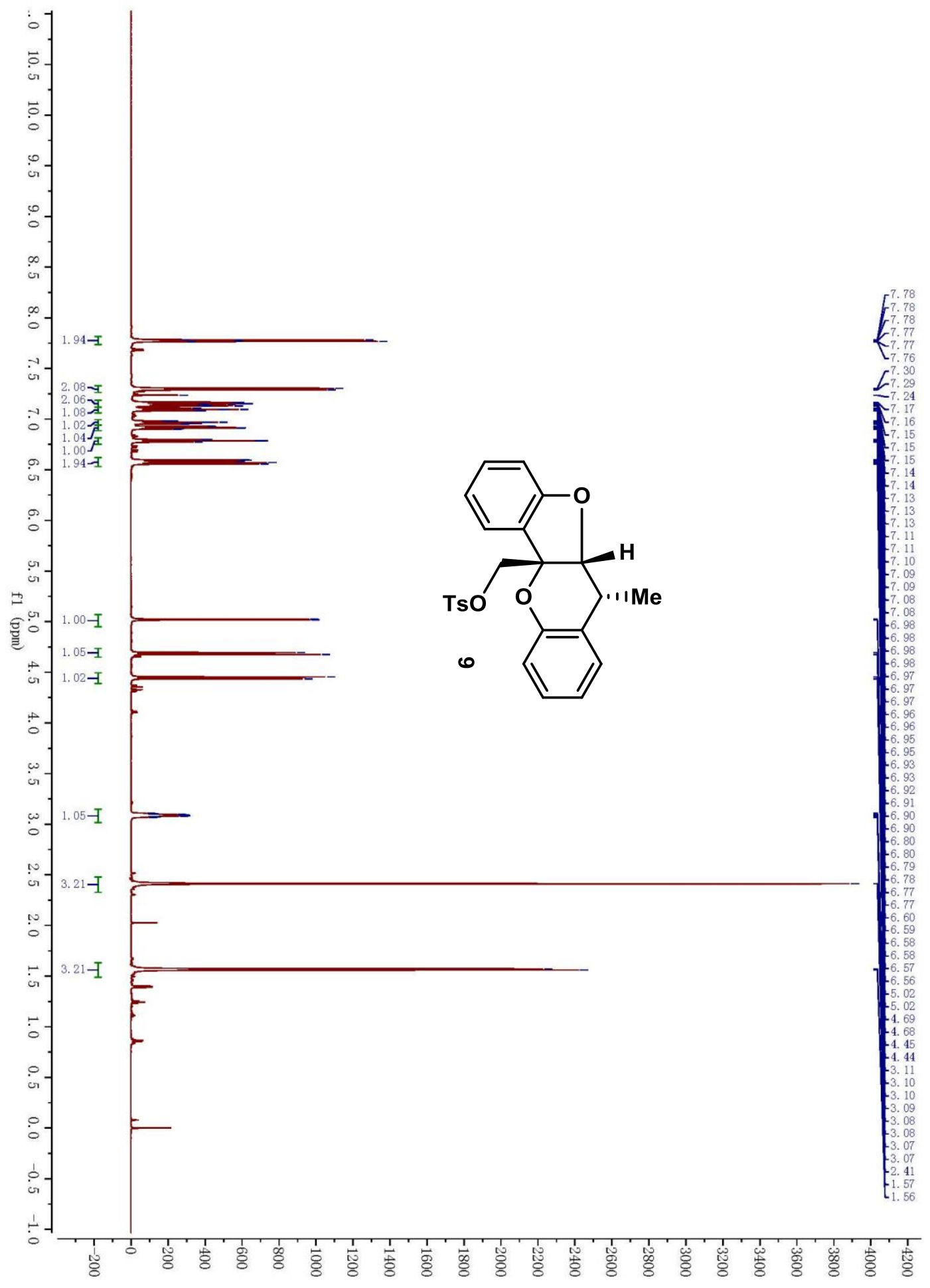




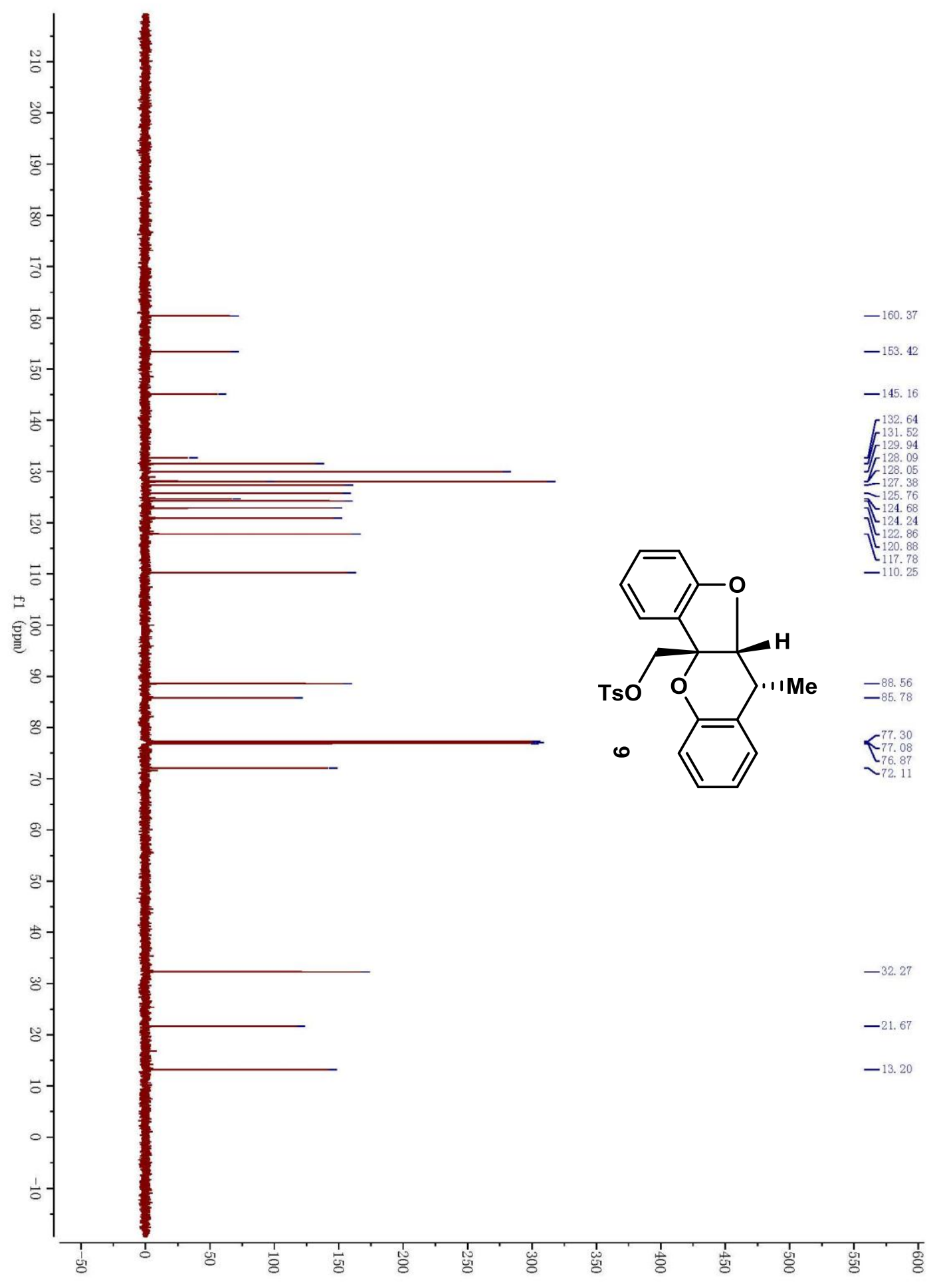




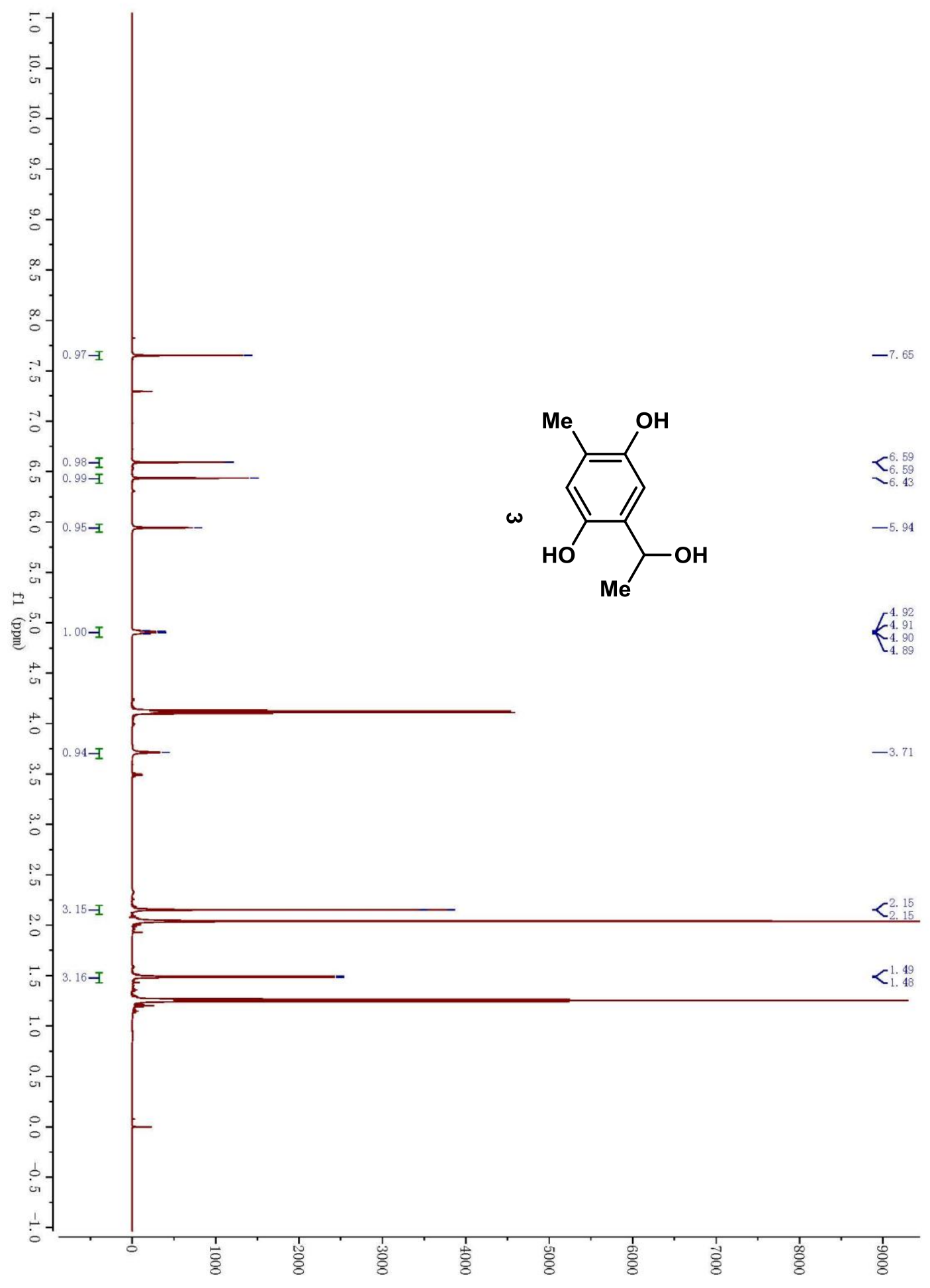




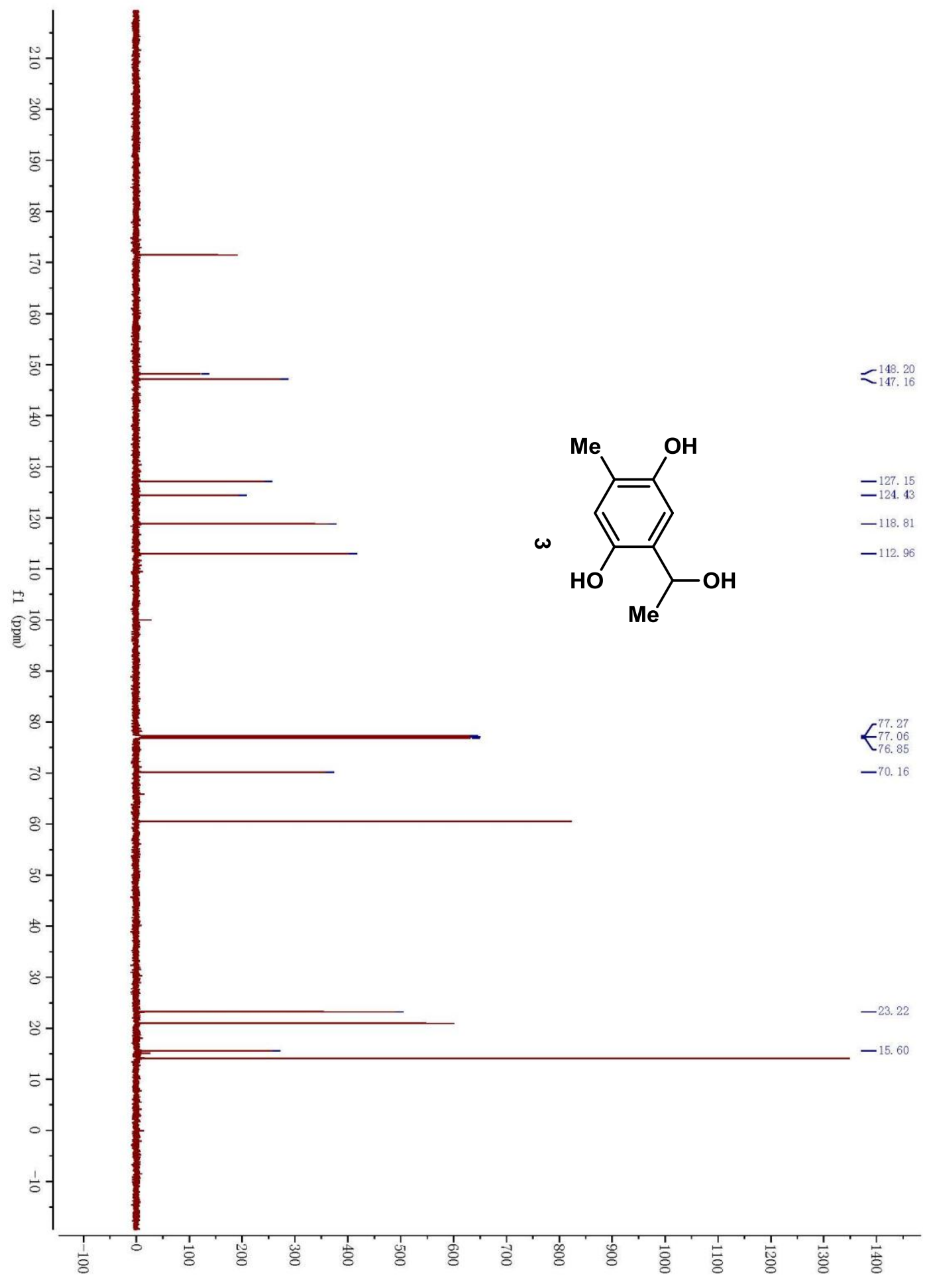




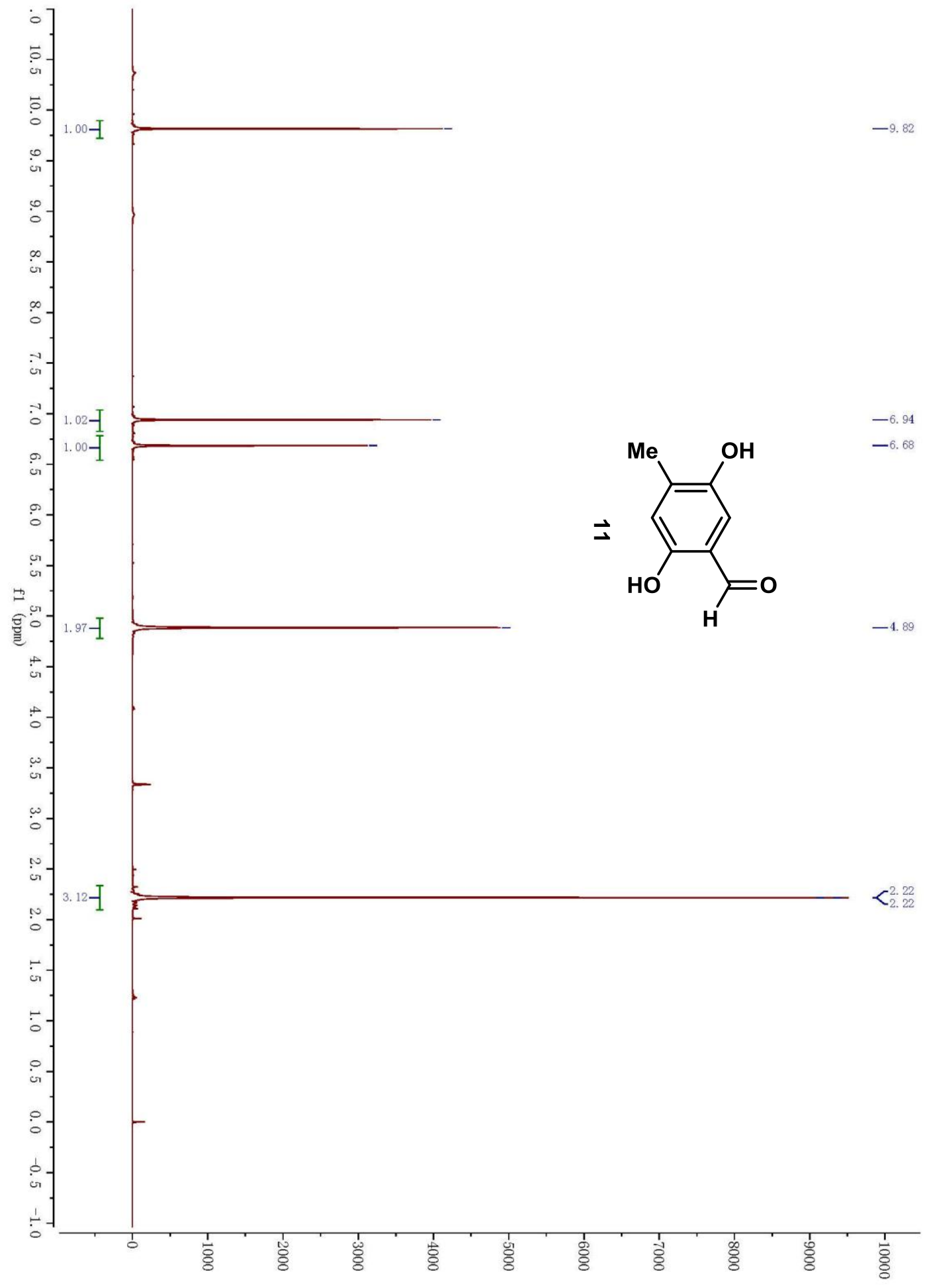




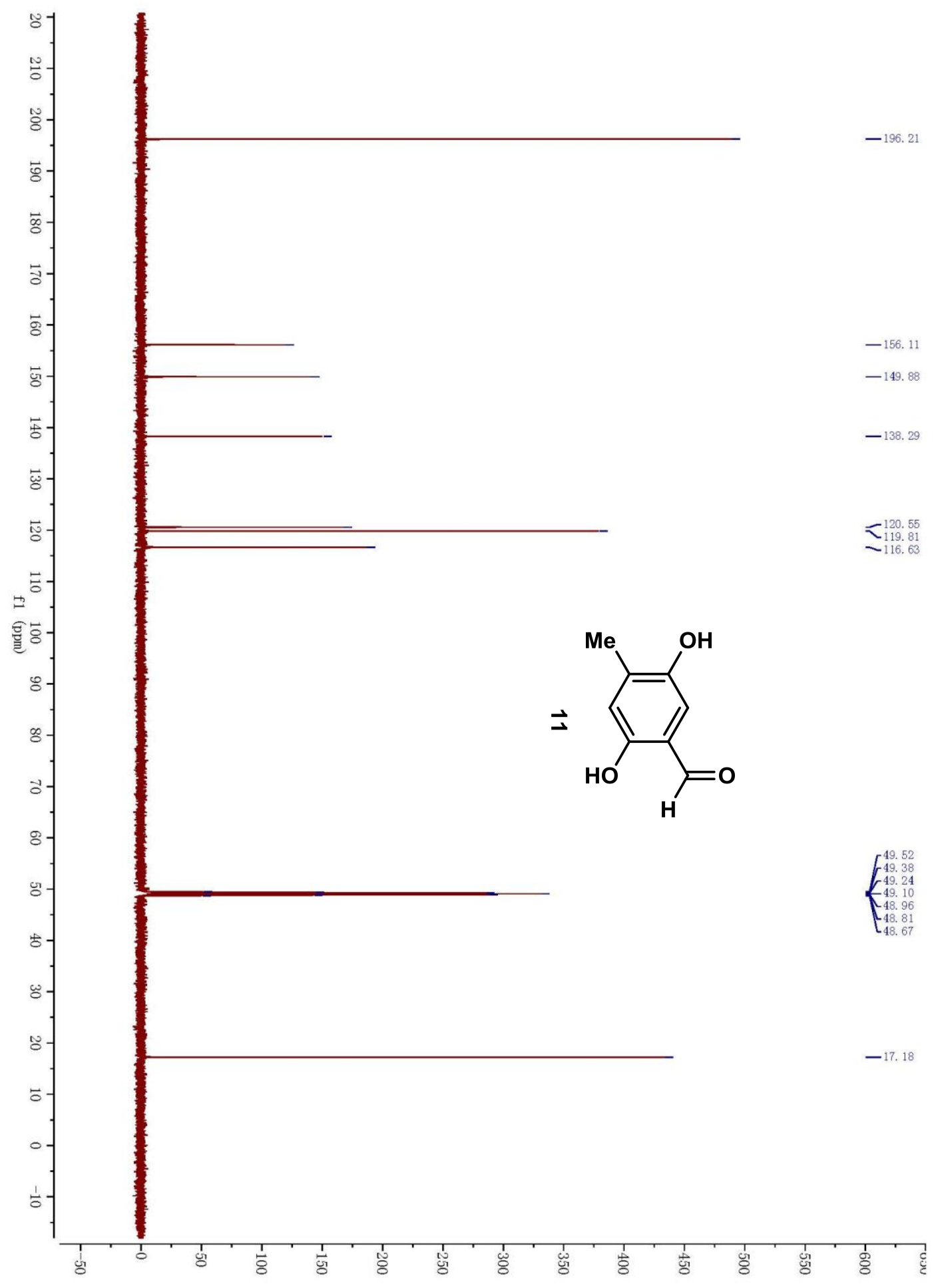




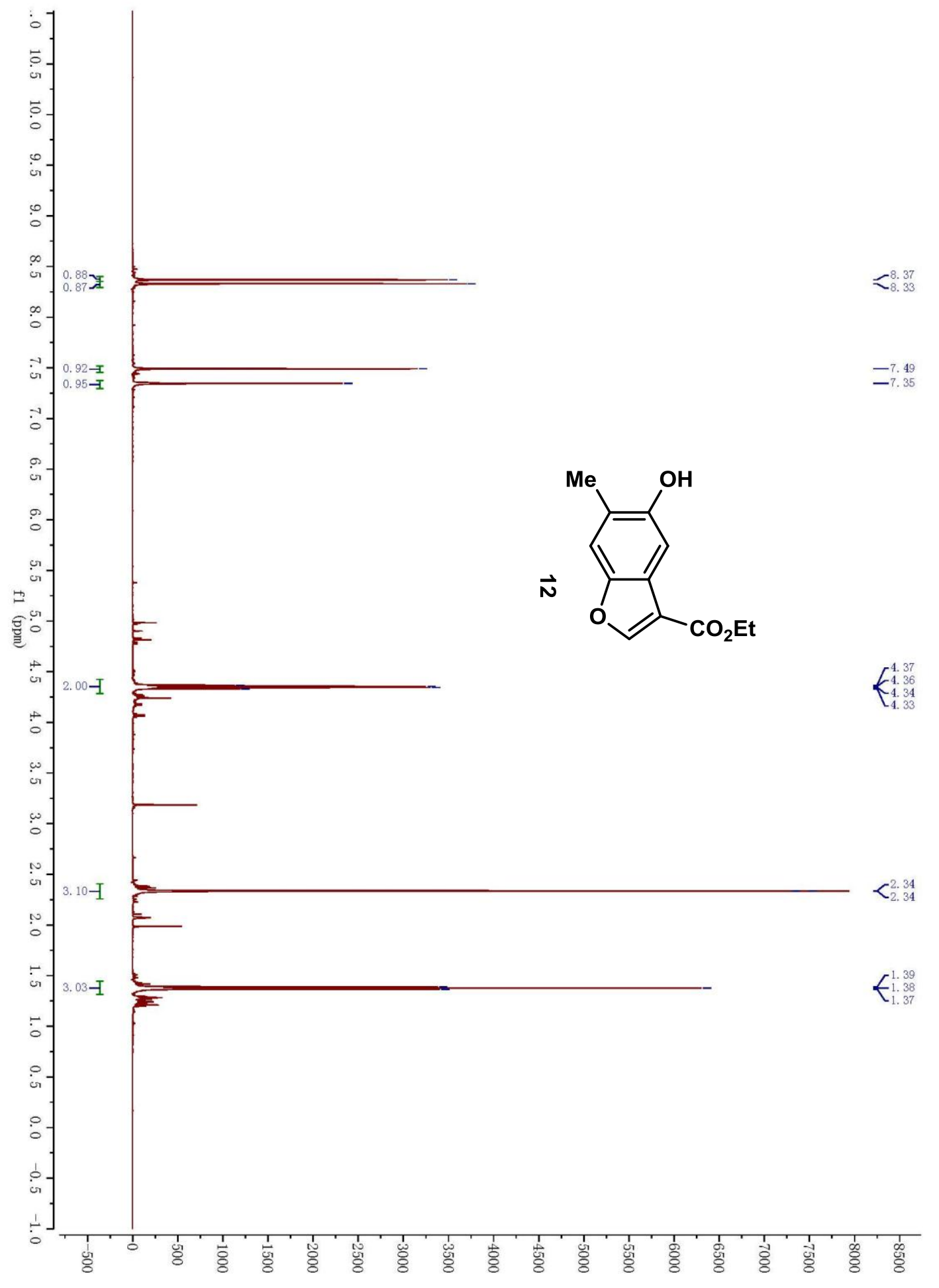




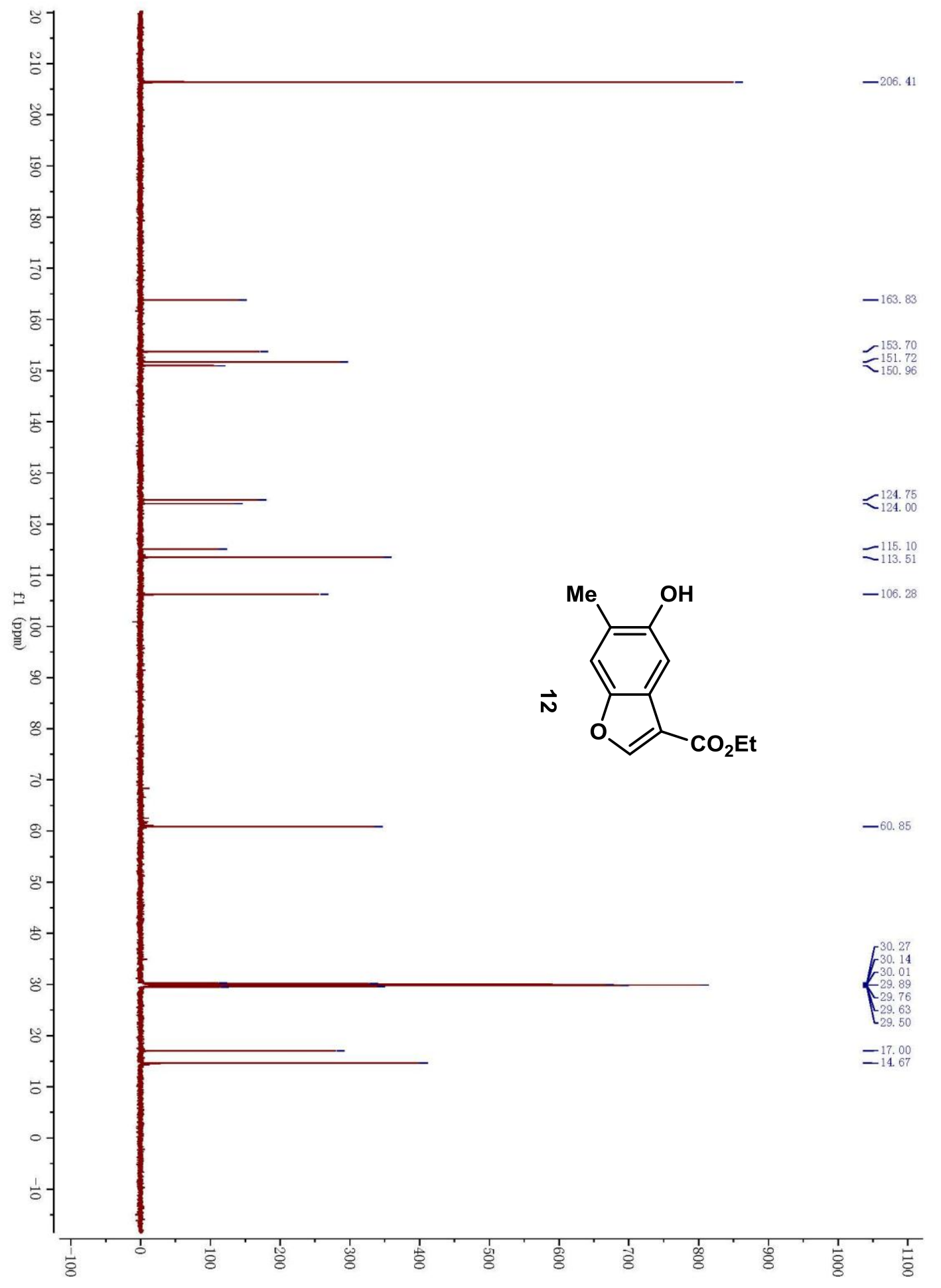




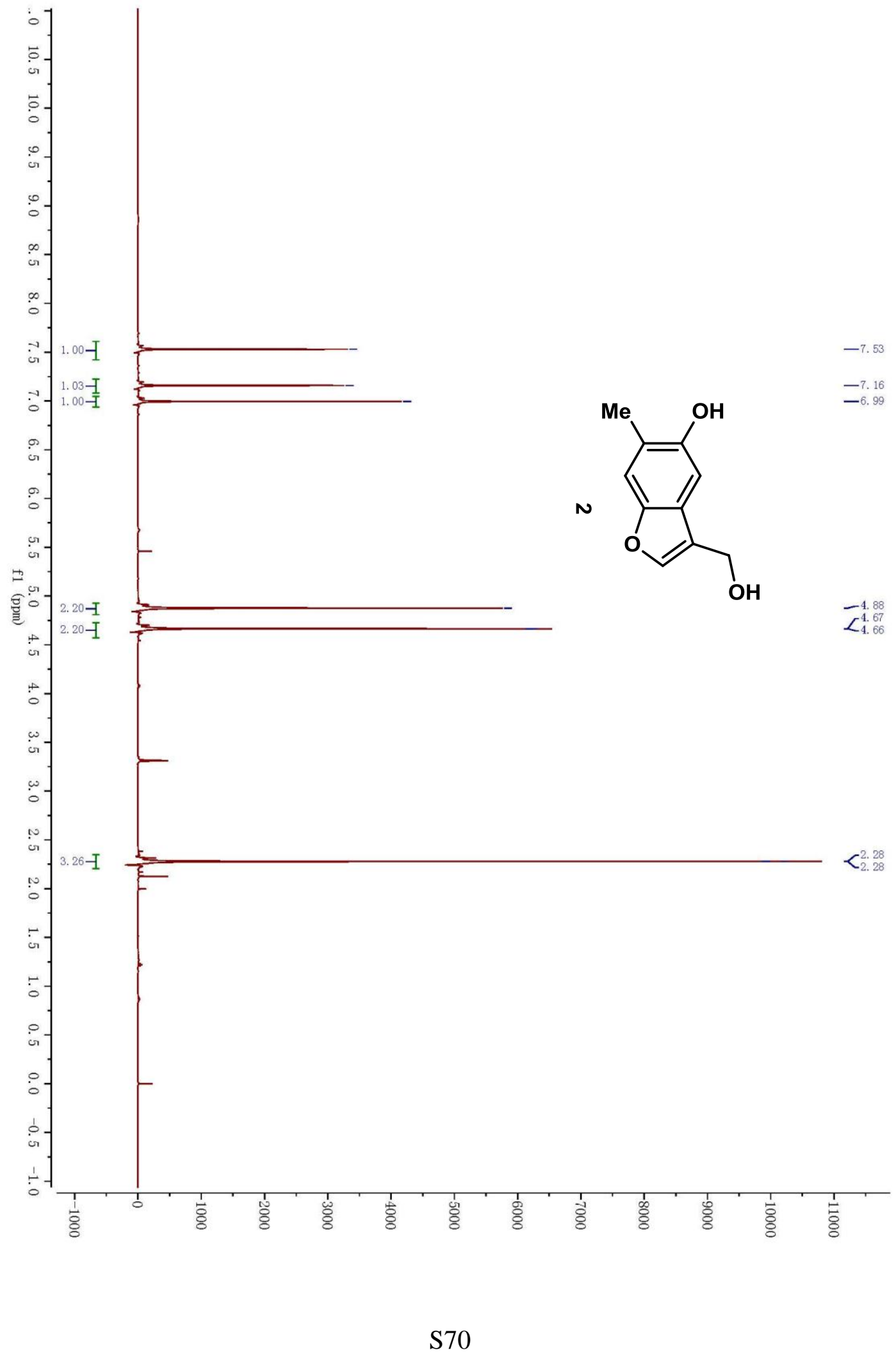




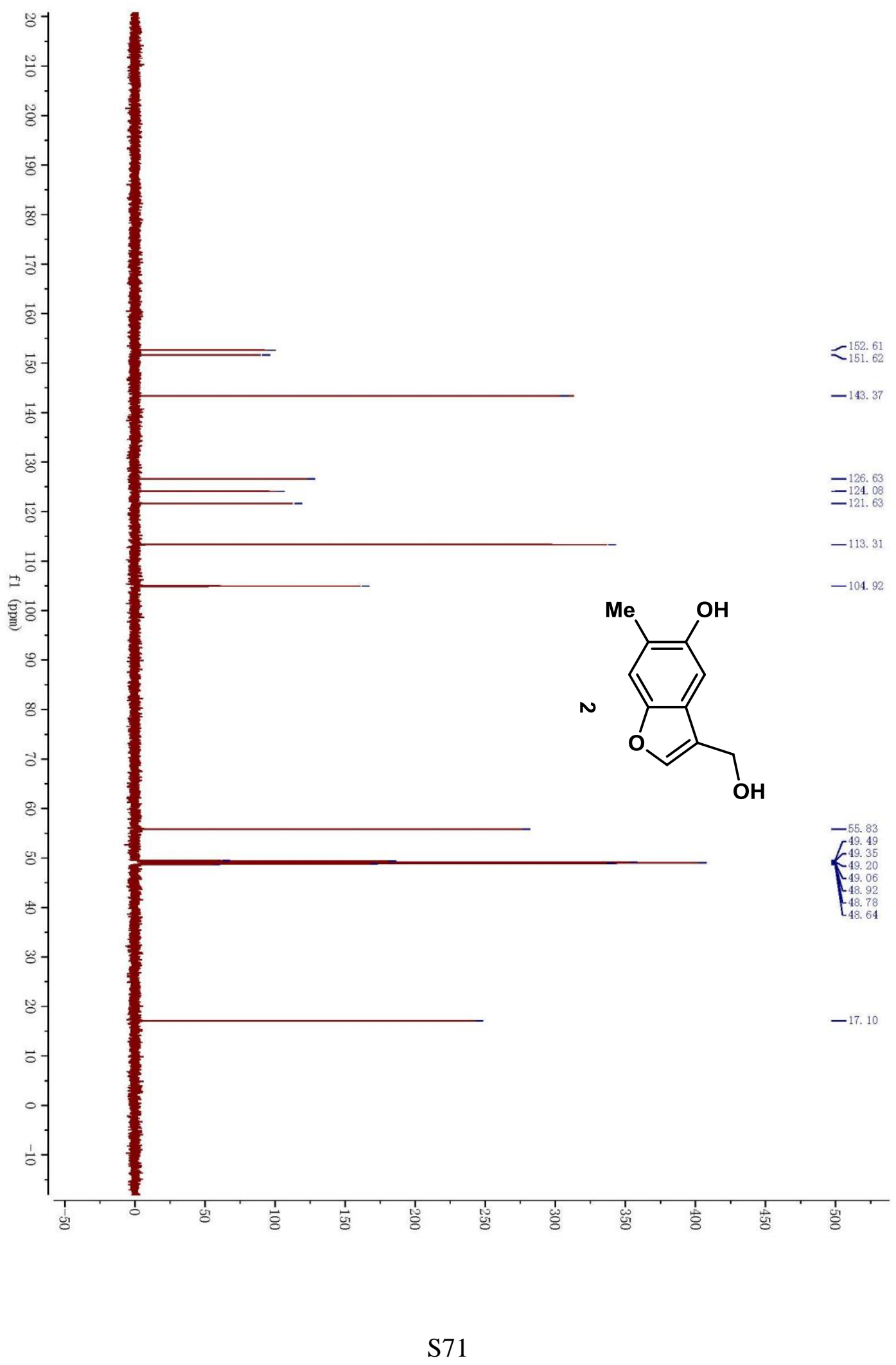




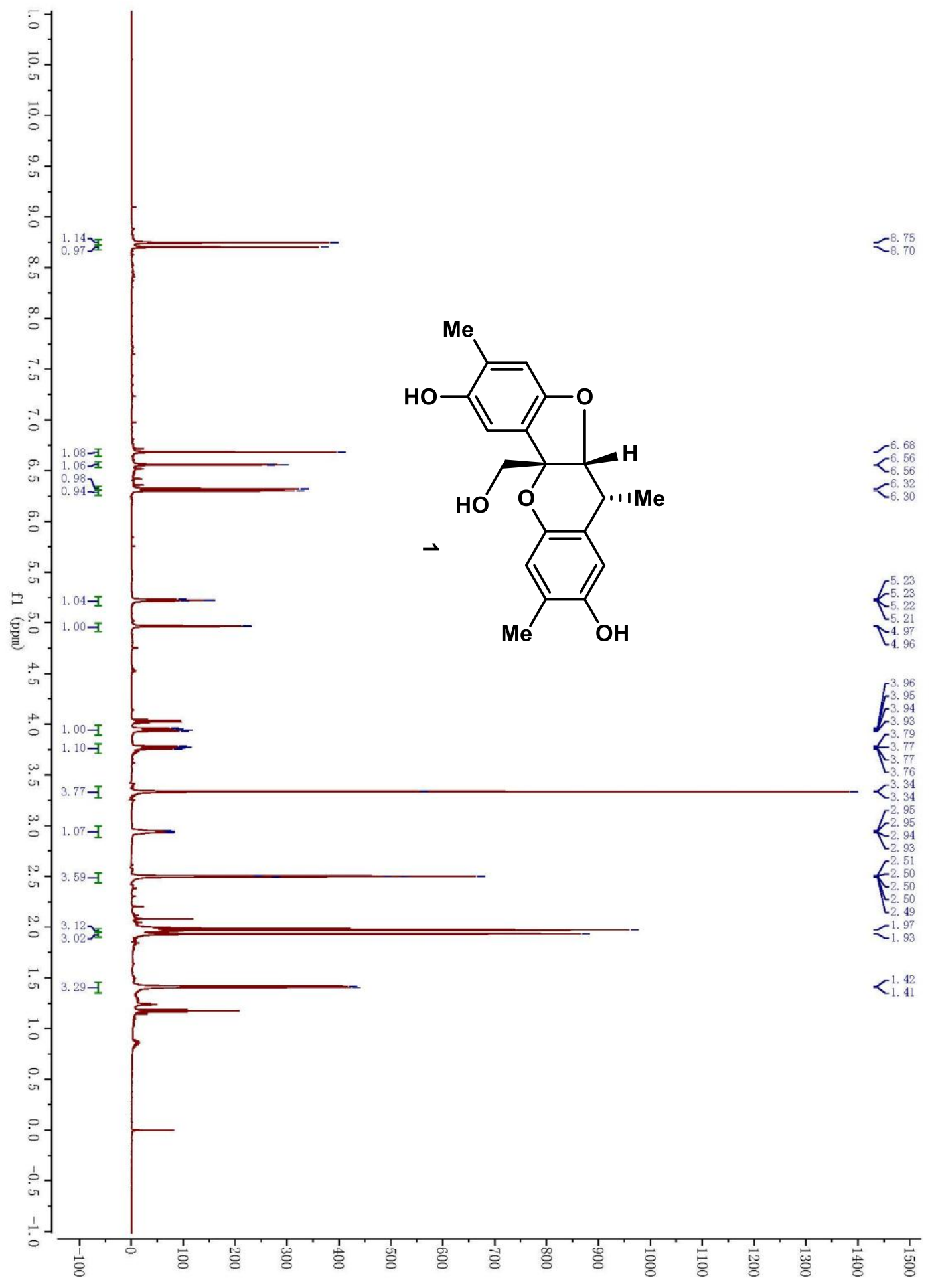




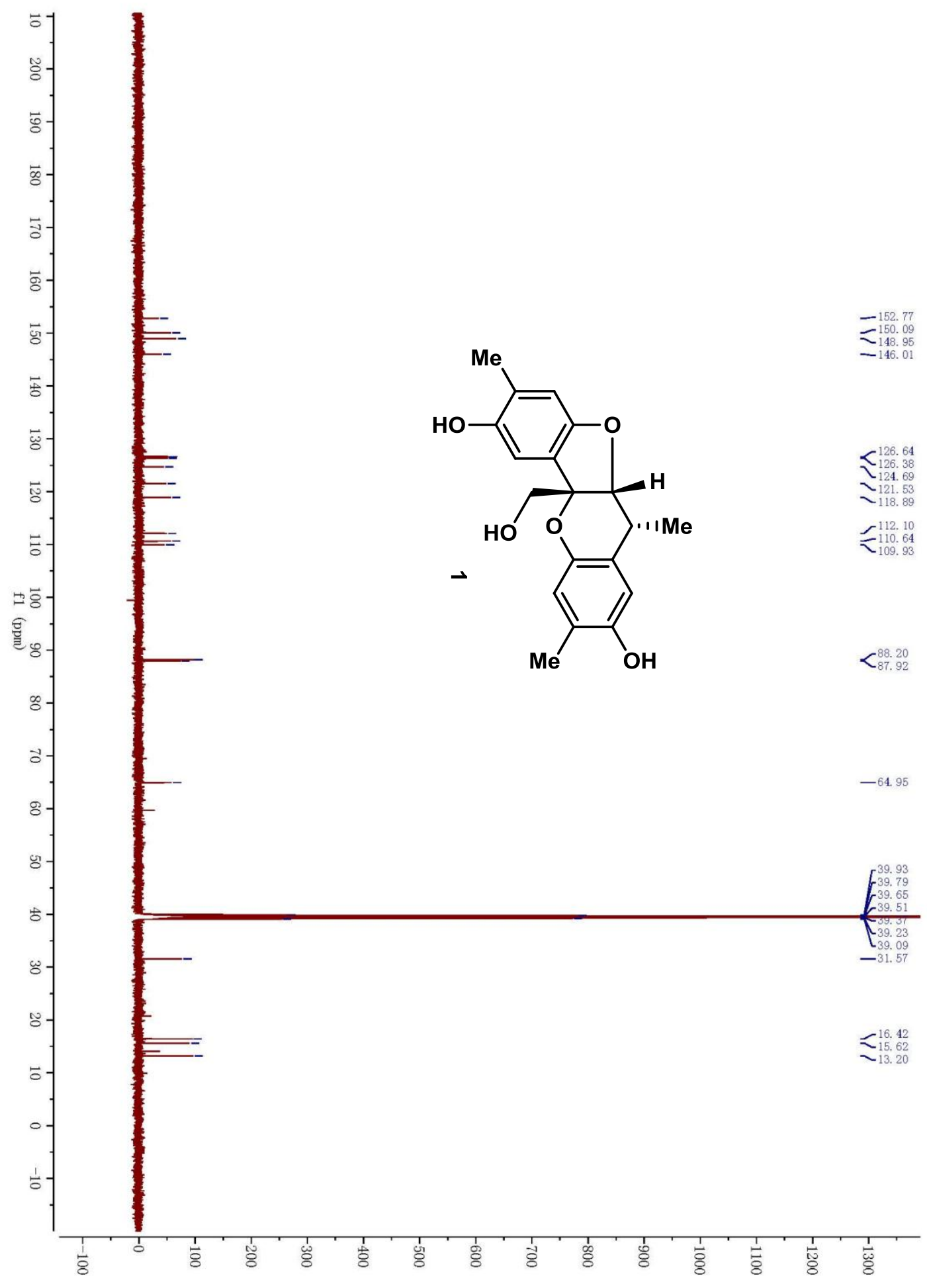

